\title{
Performance of Multirate CDMA Transmission in Cellular Environment
}

by

\section{Mahbubul Alam}

\author{
A thesis submitted to \\ the Faculty of Graduate Studies and Research \\ in partial fulfillment of \\ the requirements for degree of \\ Master of Engineering
}

\author{
Ottawa-Carleton Institute for Electrical Engineering \\ Faculty of Engineering \\ Department of Systems and Computer Engineering \\ Carleton University \\ Ottawa. Ontario \\ January 1998 \\ (C) 1998, Mahbubul Alam
}


National Library

of Canada

Acquisitions and

Bibliographic Services

395 Weillington Street

Ottawa ON K1A ONA

Canada
Bibliothèque nationale

du Canada

Acquisitions et

services bibliographiques

395. rue Wellington

Ottawa ON K1A ON4

Canada
The author has granted a nonexclusive licence allowing the National Library of Canada to reproduce, loan, distribute or sell copies of this thesis in microform, paper or electronic formats.
L'auteur a accordé une licence non exclusive permettant à la Bibliothèque nationale du Canada de reproduire, prêter, distribuer ou vendre des copies de cette thèse sous la forme de microfiche/film, de reproduction sur papier ou sur format électronique.

L'auteur conserve la propriété du droit d'auteur qui protège cette thèse. $\mathrm{Ni}$ la thèse ni des extraits substantiels de celle-ci ne doivent être imprimés ou autrement reproduits sans son autorisation. 


\section{Abstract}

The existing forms of wireless communications continue to experience rapid growth in the recent years, and new applications and approaches are being spawned at an unprecedented rate. A lot of research work has been done for the upcoming PCS which will provide many services to the users through a single handset. One of the access schemes which is actively considered for PCS is CDMA.

This thesis considers a CDMA overlaid cellular system, containing microcells overlaid over large macrocells, in order to enhance capacity and to provide multirate services. This architecture has the advantage that it does not require a large number of handoffs which are necessary in microcellular systems. Also, the users in a microcell can accommodate higher data rates because of the smaller delay spread in microcells. The use of CDMA is suitable in such an architecture as it has the ability to handle different data rates by varying the chip rates. that is the transmission bandwidth is different for different data rate users.

In communication systems, the incoming interference power is proportional to the bandwidth of the signal. In this thesis, due to multirate data transmission, the variable bandwidth present in a CDMA system is exploited to enhance the performance of the system. A fractionally spaced decision feedback equalizer is used at the receiver section to achieve the spectral coherence. As the chip rates are different, therefore, a chip spaced equalizer provides improved performance in the presence of additive noise and interference coming from different data rate users. It is seen that performance is dependent on the number and combination of interferers at different data rates. Average power control, equal bit energy and imperfect power control criteria is considered. Up to the extent of this work, it is found that the performance of varying chip rate is superior to that of the fixed chip rate case. 
to my parents.......... 


\section{Acknowledgments}

I wish to express my gratitude to my thesis supervisor, Professor A.U.H. Sheikh. for suggesting the topic of this thesis and his continuous guidance during the research. I am particularly grateful to him for giving me freedom for queries and help me to explore ideas.

I am also grateful to Professor David D. Falconer for his cooperation during the submission and arrangement of defence of the thesis.

I would like to thank Dr. Mohammed El-Tarhuni for showing patience while listening to my ideas and interesting discussions that followed. I also like to thank Shahid R. Chaudry for helping me with computer software.

I thank all the students in the PCS laboratory for putting up a nice working environment. Moreover. I am appreciative of the faculty and staff of Carleton University and the department of Graduate Studies and Research who provided much support through the development of my thesis.

Finally. I admire to my family for their encouragement and support throughout the duration of this research. 


\section{Table of Contents}

Abstract . . iii

Acknowledgment $\quad \mathrm{V}$

Table of Contents $\quad$ vi

List of Figures $\quad$ ix

\section{Chapter 1}

Introduction

1.1 Thesis Motivation 1

1.2 Thesis Description 4

1.3 Thesis Structure 5

\section{Chapter 2}

Cellular Communication: Background and Related Research 6

2.1 Mobile Radio Channels 6

2.1.1 Rayleigh Fading Distribution 8

2.1.2 Average Power Delay Profile 9

2.1.3 Shadowing and Near-far effect I0

2.1.4 Fading Multipath Channel Characteristic II

$\begin{array}{lll}2.2 & \text { Multiple Access Schemes } & 13\end{array}$

$\begin{array}{lll}2.3 & \text { Multiuser DS-CDMA } & 17\end{array}$

$\begin{array}{lll}2.3 .1 & \text { Gold Codes } & 19\end{array}$

$\begin{array}{lll}2.4 & \text { Adaptive Equalization } & 21\end{array}$

2.4.1 Interference Mitigation 26

$2.5 \quad$ Overiaid Cellular Architecture 29 


\section{Chapter 3}

System Design and Description

3.1 Transmitter Model 33

3.2 Spreading Codes 34

3.3 Channel Model 38

3.4 Receiver Model 41

3.5 Simulation Scenarios 46

3.6 SIR in Multiple Data Rate Scenario 48

\section{Chapter 4}

Results

4.1 Receiver Parameters Initialization 54

4.2 Calibration of the System 59

4.3 Results for V_BW case 63

4.3.1 Equal Average Power for each user 64

4.3.I.I Desired user at H-Data rate 64

4.3.1.2 Desired user at L-Data rate 71

4.3.1.3 Desired user at Voice rate 76

4.3.2 Equal Bit energy for each user 82

4.3.2.1 Desired user at H-Data rate 82

4.3.2.2 Desired user at L-Data rate 86

4.3.2.3 Desired user at Voice rate 90

$\begin{array}{lll}4.4 & \text { Results for F_BW case } & 95\end{array}$

4.4.1 Equal Average Power for each user 95

4.4.1.1 Desired user at $\mathrm{H}$-Data rate $(\mathrm{PG}=15) \quad 95$

4.4.1.2 Desired user at Voice rate $(\mathrm{PG}=63) \quad 95$

4.5 Imperfect Average Power Control 101 


\section{Chapter 5}

Conclusion

$\begin{array}{lll}5.1 \text { Discussion } & 104\end{array}$

$\begin{array}{lll}5.2 & \text { Proposals for Future Work } & 108\end{array}$

Appendix

109

References

114

Bibliography

119 


\section{List of Figures}

Figure 2.1 Overlaid Cellular Architecture 31

Figure $3.1 \quad$ Illustration of Gold code generation 35

Figure 3.2 Normalized correlation of Gold code sequence 35

Figure 3.3 Data symbol and transmitted symbol 37

Figure $3.4 \quad$ Three ray channel model 40

Figure 3.5 Exponentially decaying power delay profile 40

Figure 3.6 Transmitter and channel model 42

Figure 3.7 Receiver structure 45

Figure 3.8 Power and energy relationship in multiple data rate 50

Figure 4.1 DFE performance as a function of number of feedforward taps 56 for a single user in F_BW case

Figure 4.2 DFE performance as a function of number of feedforward taps 56 for a single user in V_BW case

Figure 4.3 Convergence of RLS DFE (27.2) receiver in F_BW 58

Figure 4.4 Convergence of RLS DFE (27.2) receiver in V_BW 58

Figure 4.5 System performance in single user case 61

Figure 4.6 System stability in multiuser case 61

Figure 4.7 System performance in multiuser case $\quad 62$

$\begin{array}{lll}\text { Figure } 4.8 & \text { BER performance in scenario- } 1 & 65\end{array}$

$\begin{array}{lll}\text { Figure 4.9 MSE performance in scenario-I } & 65\end{array}$

$\begin{array}{lll}\text { Figure 4.10 Probability of outage due to MSE in scenario-1 } & 67\end{array}$

Figure 4.11 Probability of outage due to BER in scenario-1 67

Figure 4.12 BER performance in scenario-2 69

Figure 4.13 MSE performance in scenario-2 69

Figure 4.14 Comparison of scenario-1 and 2 in terms of BER 70

Figure 4.15 Comparison of scenario- 1 and 2 in terms of MSE outage prob. 70

Figure 4.16 BER performance in scenario-3 
Figure 4.17 MSE performance in scenario-3

Figure 4.18 Probability of outage due to MSE in scenario-3 73

Figure 4.19 Probability of outage due to BER in scenario-3 73

$\begin{array}{lll}\text { Figure 4.20 } & \text { BER performance in scenario-4 } & 74\end{array}$

$\begin{array}{lll}\text { Figure 4.21 } & \text { MSE performance in scenario-4 }\end{array}$

Figure 4.22 Comparison of scenario-3 and 4 in terms of BER 75

Figure 4.23 Comparison of scenario-3 and 4 in terms of MSE outage prob. 75

$\begin{array}{lll}\text { Figure 4.24 } & \text { BER performance in scenario-5 }\end{array}$

$\begin{array}{lll}\text { Figure 4.25 MSE performance in scenario-5 } & 77\end{array}$

Figure 4.26 Probability of outage due to MSE in scenario-5 78

Figure 4.27 Probability of outage due to BER in scenario-5 78

$\begin{array}{lll}\text { Figure 4.28 BER performance in scenario-6 } & 79\end{array}$

Figure 4.29 MSE performance in scenario-6

Figure 4.30 Comparison of scenario-5 and 6 in terms of BER 80

Figure 4.31 Comparison of scenario-5 and 6 in terms of MSE outage prob. 80

Figure 4.32 Comparison of 15 user case $\quad 81$

Figure 4.33 Comparison of 8 user case $\quad 81$

$\begin{array}{lll}\text { Figure 4.34 BER performance in scenario-l } & 83\end{array}$

Figure 4.35 MSE performance in scenario- 1

Figure 4.36 Probability of outage due to MSE in scenario-1 84

Figure 4.37 Probability of outage due to BER in scenario-1 84

Figure 4.38 Comparison of scenario- 1 and 2 in terms of BER 85

Figure 4.39 MSE performance in scenario-2 85

$\begin{array}{lll}\text { Figure 4.40 } & \text { BER performance in scenario-3 } & 87\end{array}$

$\begin{array}{lll}\text { Figure 4.41 MSE performance in scenario-3 } & 87\end{array}$

Figure 4.42 Probability of outage due to MSE in scenario-3 38

Figure 4.43 Probability of outage due to BER in scenario-3 38 
Figure 4.44 Comparison of scenario-3 and 4 in terms of BER

Figure 4.45 MSE performance in scenario-4

Figure 4.46 BER performance in scenario-5

Figure 4.47 MSE performance in scenario-5 91

Figure 4.48 Probability of outage due to MSE in scenario-5 92

Figure 4.49 Probability of outage due to BER in scenario-5 92

Figure 4.50 Comparison of scenario-5 and 6 in terms of BER 93

Figure 4.51 MSE performance in scenario-6 93

Figure 4.52 Comparison of 15 user case 94

Figure 4.53 Comparison of 8 user case 94

Figure 4.54 BER performance when desired user is with $P G=15 \quad 96$

Figure 4.55 MSE performance when desired user is with $P G=15 \quad 96$

Figure 4.56 MSE outage when desired user is with $P G=15$

Figure 4.57 BER outage when desired user is with $P G=15$

Figure 4.58 BER performance when desired user is with $\mathrm{PG}=63 \quad 98$

Figure 4.59 MSE performance when desired user is with $P G=63 \quad 98$

Figure 4.60 MSE outage when desired user is with $\mathrm{PG}=63$

Figure 4.61 BER outage when desired user is with $P G=63$

$\begin{array}{lll}\text { Figure 4.62 BER performance in scenario-l (imperfect power control) } & 102\end{array}$

$\begin{array}{lll}\text { Figure 4.63 BER performance in scenario-5 (imperfect power control) } & 102\end{array}$ 


\section{Chapter 1 \\ Introduction}

\subsection{Thesis Motivation}

The demand for cellular radio communication services has been rapidly increasing and is expected to continue to increase. This unprecedented increase in user demand has been spurred on primarily by the business sector. However, with the promise of the personal convenience and freedom through tetherless communications at relatively low cost. cellular radio communication is becoming more attractive to the general public [38]. In fact mobile cellular telephone system in some large cities are currently congested to near capacity. In U.S. alone the PCS services are projected to have nearly 31 million domestic PCS subscribers by the year 2003. Thus it is imperative that the capacity of the current systems be increased while improving their quality, reliability and potential for new user services.

Cellular system design was pioneered during the 70 s by Bell Laboratories in the United States and the initial realization was known as AMPS. In Europe, several first generation systems similar to AMPS have been deployed, including: TACS in U.K.. Italy. Spain. Austria and Ireland: NMT in Scandinavian countries; C-450 in Germany and Portugal and Radiocom in France [8]. All of them use analog transmission employing FDMA technique. However, the trend is towards mobile cellular systems based on digital communications which can utilize powerful and relatively inexpensive digital signal processing integrated circuit technology. Furthermore. digital technology has beneficial properties such as superior speech quality, improved privacy or immunity to eavesdropping, simpler control and 
supervisory signaling and a natural extension to data transmission and the ISDN. Most importantly, digital cellular radio systems have the potential to provide significantly higher capacity than existing analog cellular radio system while utilizing the same available bandwidth [38]. [22].

These first generation cellular systems are now being replaced by second generation digital systems, GSM in Europe, PDC in Japan and IS-54 standard, in North America, each based on TDMA. The narrowband TDMA cellular systems promise to improve capacity by accommodating several time multiplexed users, coding, higher trunking efficiency and more effective frequency reuse. However, under conditions of time dispersive transmission due to frequency selective fading resulting from high signaling rates and relatively narrower channel coherence bandwidth constraint, TDMA digital systems are susceptible to multipath induced ISI which must be mitigated with relatively complex adaptive equalization and/or diversity combining techniques [23], [12], [56].

Recently, there has been an intense campaign to promote wideband CDMA digital cellular system (IS-95 standard) based on spread spectrum techniques [24]. [22]. It has been claimed that CDMA cellular system offers even higher capacity than the proposed TDMA cellular systems. Work has already started for the development of third generation mobile systems which unifies the worlds of cellular, cordless, radio local loop (RLL). private mobile radio (PMR), low-end wireless LAN and paging. The idea is to provide the same type of services everywhere, with the only limitation being that the available data rate may depend on the location and load of the system. This has been the major thrust of ITU-R, which is defining FPLMTS in U.S. From the same point of view, in Europe, the long term goal is a UMTS. 
The projects Advanced TDMA (ATDMA) and Code Division Testbed (CODIT) from the R\&D in Advanced Communications Technologies in Europe follow revolutionary approaches to UMTS. The former project uses TDMA and the latter one CDMA. as the principle access technique. Both approaches look promising, with some advantage for CDMA in cellular environment [8]. For example, with CDMA, a bandwidth that is in excess of the optimum bandwidth for a given environment can be tolerated and cells of widely varying size can be accommodated with almost no penalty on spectrum efficiency. Though the path being taken toward future wireless system in U.S. is very different from that in Europe. but CDMA is still considered as one of the major access technique by Joint Technical Committee (JTC) for PCS. Standard has already been set up for hybrid TDMA/CDMA and wideband CDMA (W-CDMA). Japan has established a new standardization committee and related working groups in connection with FPLMTS works, where CDMA technologies are being studied.

Since the proposal of Universal Personal Communication System by D.C. Cox [38], a lot of new ideas have been proposed aiding evolution of the new system. but the main objectives are essentially the same. These objectives include, but are not limited to provision of secure and good quality portable communication - voice. data and video. reduction in size and weight of handset and more efficient use of frequency spectrum. The other desired attributes are high reliability, power economy and ability to provide communication from anywhere to everywhere. In PCS system, users will have the flexibility of selecting a data rate for the service they want to use. It is well known that microcells can support data rates higher than is possible in macrocells because of relatively smaller delay spread in microcells. Therefore an overlaid cellular system, with microcells overlaying macrocells, provides a practical environment to study different data rate signals. The main motivation 
behind this thesis is to see whether CDMA signals transmitted at different data rates can coexist in an overlaid cellular system.

\subsection{Thesis Description}

It is known that increasing receiver knowledge on the properties of the received signals, decreases the error probability. Therefore for certain value of BER, the capacity of a system can be increased by using any information about the desired and or undesired signal at the receiver. In the proposed system, techniques which improve the BER performance by exploiting the structure of the received signal are:

(i) In CDMA systems, knowing the users code at the receiver decreases the BER

(ii) Estimating the channel at the receiver decreases the BER.

(iii) Using time diversity increases the time redundancy of the received signal and knowledge of the receiver about transmitted signal and. thereby. decrease BER.

(iv) The cyclostationary nature of digitally modulated signal is another source of information which produces spectral redundancy and can be used to reduce BER. (v) In case of multirate transmission. variable transmission BW offered by CDMA system can also be used to reduce BER

In this thesis. a CDMA overlaid [19] cellular system having three different data rates are considered to demonstrate the performance of the system. The signal is spreaded using Gold Codes. Two system approaches have been considered- a system which has same transmission bandwidth for different data rate users and the other has different transmission bandwidth for respective data rate users. A chip spaced DFE is used to cancel the interference, arising from same and other data rate users and ISI due to multipath channels. 


\subsection{Thesis Structure}

The following chapters of the thesis are organized as follows:

Chapter 2 describes the characteristics of mobile radio channels detailing the Rayleigh fading which can occur in mobile cellular environment. Recent research on CDMA systems. adaptive equalization and interference mitigation using equalizer are also discussed. The last section describes the issues for service integration in PCS.

Chapter 3 gives the system description including the kind of modulation scheme used. channels, spreading codes, transmitter and receiver structures used in the simulation. The RLS algorithm used for updating the taps of the equalizer in receiver is described. The simulation scenarios employing multirate transmission in overlaid cellular system are also described. Finally. signal to interference ratio (SIR) of the system are discussed.

Chapter 4 gives the results of the simulations for different scenarios described in chapter 3 . The results are described first for variable transmission bandwidth case and then these results are compared with that of same transmission bandwidth case. The last section describes the performance of DFE in a near-far environment.

Chapter 5 summarizes the results of chapter 4 . Conclusions are drawn and some proposals are presented for future work.

A list of references and bibliography are available at the end of the thesis. 


\section{Chapter 2 \\ Cellular Communication: Background and Related Research}

Communication channel places limitations on the performance that can be achieved in a system. In case of wireless communication, the path between transmitter and receiver can vary from simple line of sight to one that is severely obstructed by various objects. Unlike wired channels. radio channels are extremely random and do not offer easy analysis. It is therefore necessary to select a suitable model of the channel so that computer simulations can be used to predict system performance. In this chapter a review on the channel characteristic is presented which will help in determining a suitable model in the next chapter. Other important aspects of the system such as multiple access scheme. interference mitigation and need for service integration are also discussed.

\subsection{Mobile Radio Channels}

Due to multiple reflections from various objects, the radio signals travel along different paths of various lengths. Propagation models have traditionally focused on predicting the average received signal strength at a given distance from transmitter, as well as the variability of the signal strength in close spatial proximity to a particular location. Propagation models that predict the mean signal strength for an arbitrarily transmitter receiver separation distance are useful in estimating the radio coverage area of a transmitter and are called large scale propagation models. On the other hand, propagation models that characterize the rapid fluctuations of the received signal strength over very short distances or 
time duration are called small scale fading models. In small-scale fading, the received signal power may vary 30 to $40 d B$ when the receiver is moved by only a fraction of a wavelength. Due to multiple reflections, the result of lengthening of the transmitted signal is called the delav spread. If a base station transmits an impulse signal $\delta(t)$ to the mobile unit. then due to multipath scattering, the lowpass signal received at the mobile is given by [59]

$$
r(t)=\sum_{l} \alpha_{l}(t) e^{j \phi_{l}(t)} \delta\left(t-\tau_{l}(t)\right)
$$

where $\alpha_{l}(t)$ is the attenuation factor for the signal received on the $l$-th path. $\phi_{l}(t)$ is the time varying phase corresponding to the $l-t h$ path and $\tau_{l}(t)$ is the propagation delay of the l-th path. As the number of scatters increase the received discrete impulses becomes continuous pulse with a pulse length $T_{m}$ equal to the composite delay spread. The delay spread dictates the time when the next pulse can be transmitted in order to avoid ISI. It is required that the signalling rate should be much less than $I / T_{m}$ to prevent ISI in a Rayleigh fading environment. The delay spread is generally larger in urban areas than in suburban areas. The rms delay spread at $910 \mathrm{MHz}$ in an urban area (worst case San Francisco) can be upto $10-25 \mu . s[30]$ while the rms delay spread for the suburban area is (average typical case) 200-310 ns [51].

Since the received signal in a multipath channel consists of a series of attenuated. time delayed, phase shifted replicas of the transmitted signal, the baseband impulse response of a multipath channel can be expressed as [59]

$$
h(\tau ; t)=\sum_{l=0}^{N-1} \alpha_{l}(t) \exp \left[j 2 \pi f_{c} \tau_{l}(t)+\theta_{l}(t)\right] \delta\left(t-\tau_{l}(t)\right)
$$


where $\alpha_{l}(t)$ are the real amplitudes, $\tau_{l}(t)$ are the excess delays which is defined as the relative delay of the l-th multipath component as compared to the first arriving component. The phase term $2 \pi f_{c} \tau_{l}(t)+\theta_{l}(t)$ represents the phase shift due to free space propagation of the l-th multipath component plus any additional phase shift which are encountered in the channel. $N$ is the total possible number of multipath components and $\delta\left({ }^{\circ}\right)$ is the unit impulse function. Expression 2.2 may be simplified as [59].

$$
h(\tau: t)=\sum_{l} \alpha_{l}(t) e^{j \phi_{l}(t)} \delta\left(t-\tau_{l}(t)\right)
$$

where $\phi_{l}(t)$ is the overall phase shift of a single multipath component within $l-t h$ excess delay. As seen from expression 2.3, the amplitude variations in the received signal are due to time varying multipath characteristics of the channel. Depending upon the type of the scatters the envelope of the channel can be Rayleigh distributed resulting in Rayleigh fading channel.

\subsubsection{Rayleigh Fading Distribution}

In mobile radio channels, the Rayleigh distribution is commonly used to describe the statistical time varying nature of the received envelope of a flat fading signal. or the envelope of an individual multipath component. In Rayleigh faded channel. no line of sight component arrives at the receiver. The signal component arriving at the receiver through different paths have complex signal strength given by

$$
\alpha_{l}(t) e^{j \phi_{l}(t)}=X+j Y
$$

As the number of signal components arriving at the receiver is large so the central limit theorem suggests that $X$ and $Y$ are Gaussian distributed. Since $X$ and $Y$ are uncorrelated so they are also independent. In the absence of LOS component the mean of these Gaussian 
random variables is zero and the variance of each of Gaussian distributed variable is $\sigma^{2}$.

The envelope $\alpha_{l}(t)=\sqrt{X^{2}+Y^{2}}$ is a random variable with pdf given by [60]

$$
p_{\alpha_{i}(l)}(r)=\frac{r}{\sigma^{2}} e^{-r^{2} 2 \sigma^{2}} . \quad r \geq 0
$$

which is known as Rayleigh distribution. Here $\sigma$ is the rms value of the received voltage signal before envelope detection and $\sigma^{2}$ is the time average power of the received signal before envelope detection. The phase $\varphi_{l}(t)$ is a function of path length.

\subsubsection{Average Power Delay Profile}

For $N$ power delay profiles the average power delay profile $P\left(\tau_{l}\right)$ is given by [50]

$$
P\left(\tau_{l}\right)=\frac{1}{N} \sum_{i=1}^{N} p_{i}\left(\tau_{l}\right)=\left\langle\left|h\left(\tau_{l} ; t\right)\right|^{2}\right\rangle
$$

where $p_{i}\left(\tau_{l}\right)$ is the $i$-th power delay profile. All the profiles in a given profile set have the same specified delay $\tau_{1}$. The average excess delay $\bar{\tau}$ is given by [59]

$$
\bar{\tau}=\frac{\sum_{l=1}^{M} \tau_{l} P\left(\tau_{l}\right)}{\sum_{l=1}^{M} P\left(\tau_{l}\right)}-\tau_{A t}
$$

where $M$ is the index of the last sample along the delay axis which has significant amplitude and $l=I$ is a point before the minimum arrival delay $\tau_{. A}$ at which the amplitude of profile is insignificant. The location of samples at $l=l$ and $M$ is selected in such a way that moving the sample at $l=l$ to smaller delays and samples at $l=M$ to larger delays does not affect the value of $\bar{\tau}$. The rms delay spread is the square root of second central moment of 
the power delay profile and is given by

$$
\begin{aligned}
\tau_{r m s} & =\sqrt{\overline{\tau^{2}}-(\bar{\tau})^{2}} \\
\text { where, } \overline{\tau^{2}} & =\frac{\sum_{l=1}^{M}(\tau-\tau)^{2} P\left(\tau_{l}\right)}{\sum_{l=1}^{h} P\left(\tau_{l}\right)}
\end{aligned}
$$

Equation 2.7 to 2.9 do not rely on the absolute power level of $P(\tau)$. but only the relative amplitudes of the multipath component within $P(\tau)$. The average excess delay at a location in New York is reported to be $1.9 \mu \mathrm{s}$ with the average absolute delay equal to $3.6 \mu \mathrm{s}$. The rms delay spread is reported to be $2.53 \mu s$ [50].

\subsubsection{Shadowing and Near-Far Effect}

Both theoretical and measurement-based propagation models indicate that average received signal power decreases logarithmically with distance [14]. Measurements have shown that at any value of $d$, the path loss $P L(d)$ at a particular location is random and distributed log-normally (normal in $d B$ ) as follows [38]:

$$
P L(d)=\overline{P L}(d)+X_{\sigma}
$$

where $X_{\sigma}$ is a zero mean Gaussian distributed random variable (in $d B$ ) with standard deviation $\sigma$ (in $\mathrm{dB}$ ). The average large scale path loss for an arbitrarily transmitter-receiver ( $T$ R) separation is expressed as:

$$
\overline{P L}(d B)=\overline{P L}\left(d_{0}\right)+10 \log n\left(\frac{d}{d_{0}}\right)
$$

where $n$ is the path loss exponent, $d_{0}$ is the reference distance, $d$ is the T-R separation distance. The log-normal distribution describes the random shadowing effects which occur a 
large number of measurement locations which have the same T-R separation but have different level of clutter on the propagation path. This phenomenon is referred to as log-normal shadowing [59]. A cellular area may contain a number of shadow zones which extend over large area. As the mobile enters a shadow zone the mean signal strength decreases. increasing again as the mobile comes out of it.

In the CDMA scheme, all traffic channels are sharing one radio channel: some mobile units are close to the base station and some are not. Therefore a strong signal received from a near in mobile unit is likely mask the weak signal from a far end mobile unit- this is known as the near-far problem [22]. [24]. This intolerable situation may be mitigated by using power control on the reverse link to reduce the transmission power of a close-in user so that the resource can be shared equally, or else CDMA will be unusable [22]. [24]. [41].

\subsubsection{Fading Multipath Channel Characteristic}

The impulse response of the channel $h(\tau: t)$ is assumed to be wide sense stationary. so the autocorrelation function of the impulse response is defined as

$$
\varphi_{h}\left(\tau_{1}, \tau_{2} ; \Delta t\right)=\frac{1}{2} E\left[h^{*}\left(\tau_{1} ; t\right) h\left(\tau_{2} ; t+\Delta t\right)\right]
$$

In mobile radio environment the attenuation and phase shift of the channel associated with one path delay is uncorrelated with the attenuation and phase shift associated with another path delay. This type of scattering is called uncorrelated scattering. The uncorrelated scattering assumption gives

$$
\frac{1}{2} E\left[h^{*}\left(\tau_{1} ; t\right) h\left(\tau_{2} ; t+\Delta t\right)\right]=\varphi_{h}\left(\tau_{1} ; \Delta t\right) \delta\left(\tau_{1}-\tau_{2}\right)
$$

This type of model is known as wide sense stationary uncorrelated scattering model. 
When $\Delta t=0$ in the above equation then the autocorrelation function $\varphi_{h}(\tau ; 0)=\varphi_{h}(\tau)$ is the average power of the channel as a function of delay $\tau$. The parameter $\varphi_{h}(\tau)$ is known as the delay power spectrum of the channel. The range of values of $\tau$ for which $\varphi_{h}(\tau)$ is nonzero is called the delay spread of the channel $T_{m}$.

The Fourier transform of the delay power spectrum is denoted by $\varphi_{h}(\Delta f: \Delta t)$ and is known as the spaced frequency spaced time correlation function of the channel. When $\Delta t=0$ then $\varphi_{h}(\Delta f)$ defines the frequency coherence of the channel. This Fourier transform relationship between $\varphi_{h}(\Delta f)$ and $\varphi_{h}(\Delta t)$ suggests that the coherence bandwidth of the channel is given by reciprocal of the delay spread i.e.

$$
(\Delta f)_{h}=\frac{1}{T_{m}}
$$

When frequency separation between two signals is greater than $(\Delta f)_{h}$ they are affected differently by the channel. If $(\Delta f)_{h}$ for a channel is small compared to the bandwidth of the information signal transmitted, then the channel is said to be frequency selective i.e.

$$
W \gg(\Delta f)_{h}
$$

where $W$ is the bandwidth of information signal. If $W=1 / T$ where $T$ is the signalling interval then the frequency selective channel implies that

$$
\begin{aligned}
& \frac{1}{T} »(\Delta f)_{h} \\
& \text { or } T \ll T_{m}
\end{aligned}
$$

When $\Delta t$ is nonzero, the time variation in the channel are a measure of Doppler shift. Consider that $S_{h}(\Delta f ; \lambda)$ represents the Fourier transform of $\varphi_{h}(\Delta f: \Delta t)$ with respect to 
$\Delta t$. When $\Delta f=0$ then $S_{h}(\Delta f ; \lambda)=S_{h}(\lambda)$ which is called the Doppler power spectrum of the channel. The range of values of $\lambda$ for which $S_{h}(\lambda)$ is nonzero is called the Doppler spread of the channel denoted by $B_{d}$. The coherence time $(\Delta t)_{h}$ of the channel is given by the reciprocal of $B_{d}$. When channel is time invariant i.e. $\varphi_{h}(\Delta t)=1$ then $S_{h}(\lambda)$ is equal to delta function $\delta(t)$. So a slowly fading channel has a larger coherence time and hence smaller Doppler spread. It should be noted that the criteria that defines frequency nonselective fading. $T » T_{m}$, makes ISI negligibly small. In this case the symbol energy extends only over a small leading portion of the next symbol interval. So in frequency nonselective case, there is a duration $T-T_{m}$ for each received symbol which is free of intersymbol interference as it contains energy of that symbol only.

In [3]. the influence of channel estimation of a coherent CDMA system have been analyzed. The analysis allows predicting the system's performance, optimizing the parameters and helps to compare different system designs with respect to their performance. It was found that the noncoherent system design is able to beat the performance of coherent system for data channel BER's in the range $10^{-2}$ to $10^{-3}$.

\subsection{Multiple Access Schemes}

Three access technologies are currently in common use in the wireless systems namely FDMA, TDMA and CDMA. Until 1990 FDMA was the most prevalent access scheme where the entire bandwidth is divided into channels and these channels are distributed among the cells using a certain frequency reuse pattern. Currently the channel bandwidth of $30 \mathrm{kHz}$ is mostly used. To use higher data rate services channels with higher bandwidth 
are needed, so certain channels have to be assigned which have a different bandwidth. However such a scheme is spectrally inefficient, therefore, it can be assumed that in FDMA a scheme to integrate services having different data rates is inflexible and inefficient.

TDMA system maintains the orthogonality of channels by separating them in time. If three channels are multiplexed in one channel bandwidth of an FDM system then the system capacity increases by three times, however higher sampling rates are required to achieve this time orthogonality. TDMA system also has the inherent capability of integrating different data rate services by demanding multiple slots in a frame thus increasing the bandwidth. This approach is used in second generation mobile systems like GSM and IS54. The main drawback of TDMA is the requirement of accurate timing clock. TDMA transmission also undergoes severe time dispersion. However the use of diversity schemes and adaptive equalization can increase the performance of the system [56].

The use of CDMA has gained popularity in recent years. In this scheme each user in the system has a particular code with which the data is spread over a much wider band. Other users, using different codes, are also spread over the same band but do not significantly interfere with each other because of the orthogonality of codes. The receiver despreades the desired user by using the same code which was used to spread it at the transmitter. Other users however are not affected and remain spread thus decreasing the interference in the information bandwidth. The interference from other users depend on the processing gain (PG) which is defined as the ratio of spread bandwidth to the information bandwidth. The larger is the processing gain the smaller is the interference encountered for a given number of users. The spreading of the signal reduces the amplitude of the signal by the 
factor of PG so that the signal becomes more closer to the noise sequence and hence becomes difficult to intercept.

The main reasons for the use of CDMA are (i) resistance to multipath fading. (ii) linear increase in the capacity with any reduction in the interference [59] and (iii) antijamming [44]. Ideally the spreading code is completely random and has no correlation with its shifted version. So in the ideal case the correlation between two shifted versions of the code is zero. Therefore if the delay between two replicas of a signal is more than one chip period the correlation between them is ideally zero and multipath fading is effectively removed. In practice such ideal spreading codes do not exists so a spreading code that has some correlation with its shifted version resulting in intersymbol interference, but CDMA greatly reduces it. Another implication of the property of low crosscorrelation of the codes is that unlike TDMA or FDMA there is no hard limit to the number of users allowed in the system at a time. As the number of users increases, the signal quality degrades gracefully placing a soft limit on the number of users.

The capacity improvement in CDMA systems becomes evident by considering certain issues. Cellular systems cannot have the same transmission frequency in the adjacent cells because of interference problems but CDMA is inherently resistant to interference so the same spectrum can be used in every cell. This means that CDMA does not require complex frequency management schemes needed for FDMA and TDMA systems and results in increase of frequency reuse efficiency by a factor of 4-6. Next consideration is the voice activity factor. When CDMA is used to transmit voice signals, capacity can be improved by exploiting the voice activity which is typically lower than $40 \%$. In CDMA all users share the same channel, so when some of the users on that channel are not talking then all 
the other users on the channel benefit from less interference. Thus voice activity factor reduces the mutual interference by more than $60 \%$ and the capacity is increases by more than 2.5 times. Another factor which translates directly into capacity improvement is sectorization in a cell. For a $120^{\circ}$ sector, the reduced interference, than that of an omnidirectional antenna, results in three times increase in capacity. The capacity of CDMA system is given by [22]. [24]

$$
N=\frac{W}{R} \frac{1}{E_{h} / N_{0}} \frac{1}{d}(F)(G)
$$

where $N$ is calls per cell, $W$ is spread spectrum bandwidth (1.25MH=), $R$ is data rates in kbps $(9.6 \mathrm{kbps}), E_{b} / N_{0}$ is bit energy to noise power spectral density $(6 d B), d$ is voice duty cycle $(50 \%), F$ is frequency reuse factor $(60 \%)$ and $G$ is the number of sectors in cell ( 3 for $120^{\circ}$ sector). These values give a capacity of 120 CDMA channels in $1.25 \mathrm{MHz}$ bandwidth. In [22] the capacity is shown to be 108 channels in the same bandwidth for $R=8 k b p s$ and $E_{h} / N_{O}=7 d B$. This capacity is 18 times that of FDMA and 6 times that of TDMA assuming three users sharing a channel. In [2]. it is shown that in a convolutionaly coded asynchronous CDMA system operating in $63 \mathrm{GHz}$ band. a cell radius of $1 \mathrm{~km}$ can accommodate 315 to 345 users at BER of $10^{-2}$.

One major system design problem in mobile cellular communication systems particularly in case of microcells is handoff. In CDMA as every cell uses the same spectrum so the only difference in going from one cell to the other is the code sequence. Therefore handoff from one frequency to another while moving from one cell to another is not required. So CDMA systems do not require hard handoff, instead soft handoff is used [15]. 


\subsection{Multiuser DS-CDMA}

This thesis is concerned of multiuser DS-CDMA system with adaptive signal processing at the receiver in the presence of interuser interference in a multipath fading environment where the users are with different data rates. This section presents some of the research done for multiuser DS-CDMA for mobile communication.

A DS-CDMA system proposed by QUALCOMM [22] is shown to give a many fold increase in capacity over FDMA and TDMA. The system capacity is shown for ten percent of the spectrum allocated for a cellular service provider. The forward link signal in the system share a quadrature pair of Pseudo Noise (PN) codes. Time offsets from the basic codes are used to distinguish signals from different cells and sectors using the autocorrelation property of the code. Signals transmitted from a single antenna in a CDMA radio channel are distinguished at the mobile receiver using 64 chip Hadamard or Walsh codes. The transmitted information is convolutionaly encoded and scrambled. The reverse link design employs same PN sequence pair as used for the forward link. To identify signals from different mobile stations very long PN sequences are used with user address determined time offset. The information is convolutionaly encoded and combined with 64 chip Walsh functions. The Walsh functions for the reverse channel are selected in a different way from the forward link.

M.B. Pursley [48] gives criteria for selection of code sequences for phase coded DSSSMA system where an upper bound on the worst case error probability is derived. However, unless the period of code sequence is much larger than the number of users, these bounds are of no value and the maximum error probability is not a useful performance 
parameter. An alternate approach is to treat interference terms as additional noise [35]. The signal to noise ratio at the output of the correlation receiver is used as a criterion for code selection and can be computed with reasonable amount of computation. A formula is derived which can be used to determine what code sequence length. bit energy and noise density are required to achieve certain signal to noise ratio for a given number of users. Another formula derived can be used to determine the performance given by a specific code sequence.

The performance of asynchronous CDMA system is analyzed in [1] using a semi-analytical approach for more accurate estimation of BER for a specific code sequence. The proposed semi-analytical method applies Importance Sampling (IS), a modified Monte Carlo technique. It is shown that in near-far situation such an approach gives more accurate result. where the central limit theorem cannot hold and as a result. Gaussian approximation may not be accurate.

The average probability of bit error for the case of multipath environment is considered in [36]. An approximation to average probability of bit error is derived by modeling the decision statistics as Gaussian. Each user is assumed to have a number of spreading sequences one of which is used for every few bits. This reduces the correlation between two successive sequences sent. It is shown that better performance results as the spreading gain increases and the Gaussian approximation becomes more realistic with increase in the number of users. The assumption is more accurate when spreading gain is very large as for this case multiple access interference and intersymbol interference become small compared to the Gaussian noise at the receiver. 
A coherent system requires a channel estimation that is able to follow the time variant mobile radio channel. In [3], the uplink of a coherent DS-CDMA system has been investigated. The channel estimation is carried out via a control channel, which is transmitted simultaneously to the data channel. It is seen that the symbol error rate curves for BPSK with channel estimation degrade by approximately $1-2 d B$ with respect to the ideal BPSK performance (known channel). This is due to the fact that the channel estimation is not perfect. The results in [3] are obtained for four path Rayleigh faded channel with average $E_{h} / N_{0}$ and vehicle speed as the parameter.

The single most important system requirement is identified to be power control [13]. [20]. The power control scheme used at forward link of each cell reduces the interference to adjacent cells. The power control issue is more sensitive on the reverse link. If all the mobiles are transmitting at the same power then a strong signal received from a mobile near the base station will mask the weak signal from a mobile far from the base station.

The near far problem is not an inherent characteristics of CDMA systems rather it is the inability of the conventional single user receiver to exploit the structure of multiple access interference. Researcher like [31], [32], [9] have studied multiuser detectors for synchronous and asynchronous CDMA which are near far resistant under mild conditions. A decorrelating detector is presented which has the knowledge of the spreading sequence of all the users. The performance of the decorrelating detector is shown to be either the same or quite similar to the optimum linear detector depending on the energies and correlations.

\subsubsection{Gold Codes}

A requirement of the design of a spread spectrum system for multiple access system is to find a large set of spreading codes such that as they produce as little mutual interference as 
possible. The Gold codes. invented at the Magnavox Corporation, are useful because they belong to a relatively large set of codes. They are easy to generate as require only one pair of feedback tap sets. Usually the spreading codes are periodic sequences of ones and zeros with period $N$. It is convenient to represent a sequence of binary digits by a polynomial

$$
b(D)=\ldots \ldots \ldots+b_{-2} D^{-2}+b_{-1} D^{-1}+b_{0}+b_{1} D+b_{2} D^{2}+\ldots \ldots \ldots
$$

The delay operator $D$ implies that the binary symbol which multiplies $D^{j}$ occurs during the $j-t h$ time interval of the sequence. Because the code is periodic $b_{n}=b_{.+n}$ for any $n$. The discrete periodic crosscorrelation function of two codes $b(D)$ and $b^{\prime}(D)$ is defined by D.V. Sarwart and M.B. Pursley [45] as

$$
\theta_{h h^{\prime}}(k)=\frac{l}{N} \sum_{n=0}^{N-1} a_{n} a_{n+k}^{\prime}
$$

where $a_{n}=(-1)^{h_{n}}$. Let $b(D)$ and $b^{\prime}(D)$ represents a preferred pair of $m$-sequences having period $N=2^{\prime \prime}-1$. The family of codes defined by (2.29) is called the set of Gold codes for these preferred pair of $m$-sequences.

$$
b(D), b^{\prime}(D), b(D)+b^{\prime}(D), b(D)+D b^{\prime}(D), \ldots \ldots \ldots b(D)+D^{. x^{\prime}} b^{\prime}(D)
$$

Gold code sets have the property that any pair of codes in the set say $\boldsymbol{y}$ and : have a three valued crosscorrelation spectrum which takes on the values given by the following expressions.

$$
\begin{gathered}
-\frac{1}{N} t(n) \\
-\frac{\mathrm{I}}{N} \\
\frac{1}{N}[t(n)-2]
\end{gathered}
$$




$$
\text { where, } t(n)= \begin{cases}1+2^{0.5(n+1)} & \text { for } n \text { odd } \\ 1+2^{0.5(n+2)} & \text { for } n \text { even }\end{cases}
$$

Finding preferred pairs of $m$-sequences is necessary in defining sets of Gold codes. The following conditions can define a preferred pair $\boldsymbol{b}$ and $\boldsymbol{b}^{\prime}$ of $m$-sequences.

1. $n \neq 0 \bmod 4$; that is. $n$ is odd or $n=2 \bmod 4$.

2. $\boldsymbol{b}^{\prime}=\mathrm{b}[\mathrm{q}]$ where $q$ is odd and either

$$
q=2^{k}+1
$$

or

$$
q=2^{2 k}-2^{k}+1
$$

3. Greatest common divisor $(n, k)=\left\{\begin{array}{lr}1 & \text { for } \mathrm{n} \text { odd } \\ 2 & \text { for } \mathrm{n}=2 \bmod 4\end{array}\right.$

There are a total of $N+2$ codes in any family of Gold codes for a preferred pair of $m$ sequences. In addition to their advantage in generating large number of codes, the Gold codes may be chosen so that over a set of codes available from a given generator the cross correlation between the codes are uniform and bounded. Thus the Gold codes are attractive for applications in which a number of code-division-multiplexed signals are to be used. These codes have been selected by NASA for use on Tracking and Data Relay Satellite System (TDRSS) [61].

\subsection{Adaptive Equalization}

The candidate adaptive receiver strategy considered in this thesis is - directly adapting the equalizer coefficients in an iterative manner, i.e. using RLS algorithm. Equalization techniques that have been developed during the past can mitigate user data interference. How- 
ever, rapidly varying channel characteristics impose some stringent conditions on the design of the adaptive equalizer. In recent years equalization is one of the important category of interference rejection techniques used in CDMA. Equalization techniques for combating ISI on bandlimited time dispersive channels may be subdivided into two general types- linear and non-linear equalization. Associated with each type of equalizer is one or more structures for implementing and for each structure there is a class of algorithms that may be employed to adaptively adjust the equalizer parameters.

Linear equalizers work on the principle of zero forcing or minimization of MSE which is most commonly used. The minimization of the MSE results in the optimum Wiener filter solution for the coefficient vector, which may be expressed as

$$
C_{o p t}=\Gamma^{-1} \xi
$$

where $\Gamma$ is the autocorrelation matrix of the vector of signal samples in the equalizer at any given time instant and $\xi$ is the crosscorrelation between the desired data symbol and the signal samples in the equalizer. Alternatively minimization of MSE may be accomplished recursively by the stochastic gradient algorithm introduced by Widrow, called LMS algorithm. The convergence rate of LMS algorithm is slow due to the fact that there is only one parameter. known as the step size, that controls the rate of adaptation. A faster converging algorithm is obtained by employing a RLS criterion for adjustment of the equalizer coefficients, albeit computationally more complex and proportional to $M^{2}$. where $M$ is the length of the equalizer. Another algorithm called square-root RLS algorithm [23] also have a computational complexity proportional to $M^{2}$ whereas. fast RLS algorithm [46] has a computational complexity proportional to $M$. 
Nonlinear equalizers find use in applications where channel distortion is too severe for linear equalizers to handle. In particular, the linear equalizer does not perform well on channels with spectral nulls on their frequency response characteristics. In an attempt to compensate for the channel distortion, the linear equalizer places a large gain in the vicinity of the spectral null and, as a consequence, significantly enhances the additive noise present in the received signal. This noise enhancement is unacceptable when the channel experiences severe amplitude distortion. The linear equalizer is therefore not suitable for mobile radio communication where deep fades are common. The convergence rate of LMS algorithm is especially slow in channels which contains spectral nulls. whereas the convergence rate of RLS algorithm is unaffected by the channel characteristics [23]. Over the last several years, three very effective nonlinear equalization methods have been developed. They are- DFE, symbol-by-symbol detection algorithm based on maximum a posteriori probability and sequence detection algorithm based on maximum likelihood sequence estimation criterion.

The idea in DFE is that once an information symbol has been detected. the ISI that it causes on future symbols may be estimated and subtracted out prior to symbol detection. It consists of a feedforward filter (FFF) and feedback (FBF) filter. The coefficient adjustment may be performed by using any algorithm mentioned earlier. The feedforward filter of the DFE eliminates precursor ISI while the feedback filter removes the postcursor ISI by removing exactly the ISI contributed by already detected symbols to the present estimates if all the past decisions are assumed to be correct [54]. Another form of a DFE proposed by [47]. is called a predictive DFE. It also consists of a FFF as in the conventional DFE. However, the FBF in this case is driven by an input sequence formed by the difference of the output of the detector and the output of the FFF, whereas in conventional DFE 
the FBF is fed by the output of the detector. In [47], [29], it is shown that the predictive DFE performs as well as conventional DFE in the limit as the number of taps in the FFF and FBF approach infinity.

The MAP algorithm and MLSE algorithm are optimal in the sense that they minimize the probability of error. In MAP algorithm, it is the symbol error rate that is minimized whereas, in MLSE. the Viterbi algorithm minimizes the probability of a sequence error. In practice, these two algorithms provide comparable performance [60],[52]. For time dispersive channels in which ISI spans many symbols, these probabilistic algorithms become impractical due to an exponential growing computational complexity with ISI span. Nevertheless, they serve as benchmarks against which the performance of suboptimal algorithms such as the DFE or linear equalizer can be compared.

The adaptive DFE approach has been examined for radio receivers for troposcatter radios [42], microwave line of sight [43], HF [40] and indoor radios [33]. It is found that the DFE is a simpler, efficient and practical approach compared to the more complex MLSE receiver. It is shown in [63] that the performance of DFE is nearly as good as that of MLSE as long as decision error propagation is not severe. In [53] and [49] it has shown that the performance of DFE is better in the presence of multipaths because of the implicit diversity gain obtained which is larger than the ISI degradation. As a DFE removes ISI without inverting the spectrum and hence without excessive noise enhancement so it performs superior to a linear equalizer in deep fading environment. Also as the FFF eliminates all the precursor ISI, the number of forward filter taps in DFE can be reduced relative to the number of equalizer coefficients required in a linear equalizer. This results in lesser coefficient noise for the same convergence rate as the coefficient noise increases 
with the number of taps. In [7] it is shown that a decision based nonlinear equalizer is capable of tracking fast carrier phase variation.

Benvenuto and Tomba [5] showed that for large delay spread and nonlinear ISI. performance can be enhanced by the nonlinear equalizer. In [12] the authors describe the use of DFE for indoor CDMA systems. It is shown that DFE combines the advantage of Rake by exploiting the inherent diversity resulting from multipath and performs cancellation of ISI and multiaccess interference. The configuration assumes no knowledge of channel parameters. a training sequence is however used to train the equalizer and shown to perform much better than a single stage Rake receiver. The receiver is shown to accommodate a number of users equal to half the PG for a system with very low processing gain. Wilson and Cioff [17] have shown a linear equalizer to perform much better than Rake detector in multichannel environment in the presence of near far problem.

MMSE criterion for interference suppression in DS-CDMA system is considered by [12]. The schemes are compared in three user systems and PG of 32 and are shown to give low error probability no matter how strong is the interference. In [16] the authors describe a receiver for an asynchronous CDMA systems in which the receiver uses LMS algorithm for each user. The receiver is assumed to have no knowledge of signature waveforms or timing information of other users. The receiver is simulated by using a set of Gold sequences of length 31 and is shown to have considerable improvement with respect to conventional single user receiver.

It is shown [60] that the optimum receiver for a digital communication signal corrupted by AWGN consists of a matched filter which is sampled periodically at the symbol rate. If the 
received signal samples are corrupted by ISI. the symbol spaced samples are further processed by either a linear or nonlinear equalizer. In the presence of channel distortion. the match filter prior to the equalizer must be matched to the channel corrupted signal. However in practice the impulse response is unknown and consequently, the optimum matched filter to the received signal must be adaptively estimated. A suboptimal solution in which the matched filter is matched to the transmitted signal pulse may result in a significant degradation in performance. In addition, such a suboptimal filter is extremely sensitive to any timing error in the sampling of its output [57], [23]. A fractionally spaced equalizer is based on sampling the incoming signal at least as fast as the Nyquist rate. which compensates for the channel distortion in the received signal before aliasing effects occur due to symbol rate sampling. In addition, the FSE can compensate for any timing delay or any arbitrary timing phase. In effect, the FSE incorporates the functions of matched filtering and equalization into a single filter structure.

\subsubsection{Interference Mitigation}

The conventional method of interference rejection is to filter the received signal. Adaptive filters have been used for this purpose in recent years. Unfortunately a limit exists to the gain that linear time-independent filtering can provide when the interference and SOI share the same frequency band and the signal statistics change rapidly. This is often the case in applications when the adaptive filter is used for filtering digitally modulated signals. These signals have periodic statistics and is often referred to as Cyclostationary signals and Linear time-dependent (or FRESH) filtering offers significant improvement over time-independent filtering [28]. A receiver's ability to mitigate a given level of interference depends on important factors such as the number of interferers present, the transmission bandwidth and the differences between the desired signal and interference responses. 
A receiver can achieve performance gains in the presence of interference relative to the equivalent case of stationary noise by exploiting the cyclostationary property of the interference which is distinct from that of stationary noise [57], [55], [34], [25] (AWGN has statistics which are stationary).

In a mature. highly utilized cellular radio system with a large number of users distributed over relatively small cells, the dominant source of performance is the interference, while the additive thermal noise power can be relatively insignificant on the order of tens of decibels below the interference power. Consequently, improved receiver performance can be achieved by accounting for this distinctly cyclostationary interference, rather than assuming the worst case that it is additive Gaussian stationary noise. It has also been shown that an asynchronous direct sequence spread spectrum multiple access system with an optimum, i.e. maximum likelihood sequence detector, multiuser receiver consisting of a bank of filters matched to the user waveforms followed by a Viterbi decoder is not interference limited [39]. Even for the case of sufficiently powerful interference, i.e. equal energy users can still be suppressed.

In the case when the interference posses significant cyclostationarity, a reduced state sequence estimation receiver with appropriately chosen sampling phase has been shown for a digital subscriber loop application to perform better than in an equivalent stationary coloured noise [26]. Furthermore, a receiver consisting of a synchronously spaced equalizer with optimally selected sampling phase has also been shown to exhibit similar performance improvement [57]. In addition, the performance of a fractionally spaced equalizer has been shown to be relatively insensitive to the receiver sampling phase and can yield superior performance relative to the equivalent stationary noise case [57], [25]. This use of 
a fractionally spaced equalizer as a Wiener filter to mitigate the inter-user interference exploits the temporal correlation of the cyclostationary interference.

For a Wiener filter, such as fractionally spaced MMSE DFE at the receiver, the difference between cyclostationary inter-user interference and stationary noise can be viewed in terms of their second order statistics. The autocorrelation function of the receive-filtered stationary noise is completely determined by the receive filter response and is fixed in time. In complex baseband representation, the autocorrelation of the receive filtered noise is real symmetric while the interference autocorrelation is conjugate symmetric. Depending on the degree of interference cyclostationarity, the second order statistics of interference can be quite different from that of stationary noise. In one sense, the MMSE DFE can perform better in the presence of inter-user interference than in stationary noise because the DFE can achieve a smaller prediction error at the output of its feedforward filter by exploiting the correlation of the interference [56]. Furthermore. the inter-user interference characteristics vary randomly with time in a fading environment. Thus. in order for the DFE to perform better in the presence of inter-user interference than in stationary noise, it requires updated knowledge of the interference statistics.

Another. perhaps the more general way in which to view the cyclostationarity in a signal is in terms of its spectral correlation [62]. That is, a cyclostationary signal exhibits spectral coherence at integer multiples of its periodicities such as the baud rate or carrier frequency. This spectral correlation can be exploited by frequency shift filtering to reduce the effect of the cyclostationary interference [62], [55]. This frequency shift filtering performs a linear sum of the weighted frequency shifted received signal components and can be implemented as a parallel bank of filters, each consisting of a frequency shifted product 
modulator followed by an FIR filter, whose outputs are summed. Digital signals such as BPSK. MSK and ASK which possess spectral correlation between their positive and negative frequency images and thus their corresponding receivers can exploit this additional correlation by also having weighted frequencies at twice the carrier frequency plus integer multiples of the baud rate [28], [62].

In general, a frequency shift filter estimates all parameters of the desired signal. However. the baud rate sampled data sequence is of interest in a typical digital communication system. Furthermore, if the spectral correlation associated with the carrier frequency is nonexistent or is not exploited, then the receiver is simplified as a linear processor. Thus when the output of the frequency shift filter is sampled at the baud rate, this simplified structure is equivalent to, and can be implemented as a fractionally spaced FIR equalizer [27]. For a system employing $100 \%$ excess bandwidth raised cosine filtered BPSK with zero carrier offset. such a fractionally spaced equalizer has been shown to outperform a synchronously spaced equalizer in the presence of partial band amplitude modulated interference or multipath induced ISI [27]. Moreover, an LMS trained frequency shift filter (called a timedependent adaptive filter) has shown to achieve superior performance relative to a standard time-independent adaptive filter which does not exploit the signal cyclostationarity [28].

\subsection{Overlaid Cellular Architecture}

It is likely that a standard wireless integrated system will be required in near future. Therefore a flexible integrated PCS system using CDMA overlaid concept is proposed. The flexibility in the system is achieved by varying burst rate which commensurate with selected service. The same transmission bandwidth is used for all services. On selecting a low rate 
service the terminal lowers the burst rate which increases with high rate services. Thus. the integration is more flexible. The choice of spreading bandwidth is important because it ultimately defines the services.

An overlaid cellular structure combines the advantage of microcells and macrocells. It is seen that efficient channel management can increase the system capacity by many folds [6]. Microcells provides increase in capacity of the system while macrocells allow fast moving terminals without excessive handoffs thus improving communication quality. An algorithm has to be developed by which a mobile station MS chooses whether to select a microcell channel or a macrocell channel. Typically it is desired that microcell channels be assigned to stationary MS so as to avoid excessive handoffs. A mobile station may not be allowed to select a microcell channel unless it receives the signal from microcell base station BS for a certain minimum amount of time. This insures that the mobile is not moving fast enough that a microcell channel can be allocated.

CDMA has been proposed as a method to provide expanded capacity and PCS features by overlaying existing cellular systems [18] or fixed microwave service [21]. It has also been proposed to provide microcells underlaying existing cellular macrocells [10]. A structure shown in Figure 2.1 is compared in [11] for two different access schemes, packet reservation multiple access PRMA and CDMA. The performance of two access schemes in overlaid cellular environment is compared from the point of view of spectrum efficiency as the two schemes can not be compared in terms of interference probability. The authors show a better performance for PRMA for the same value of $E_{h} / N_{0}$ used for both schemes. However it is suggested that no definite conclusion can be drawn at this early stage of research as some factors (channel coding) if taken into account can change the picture completely. 


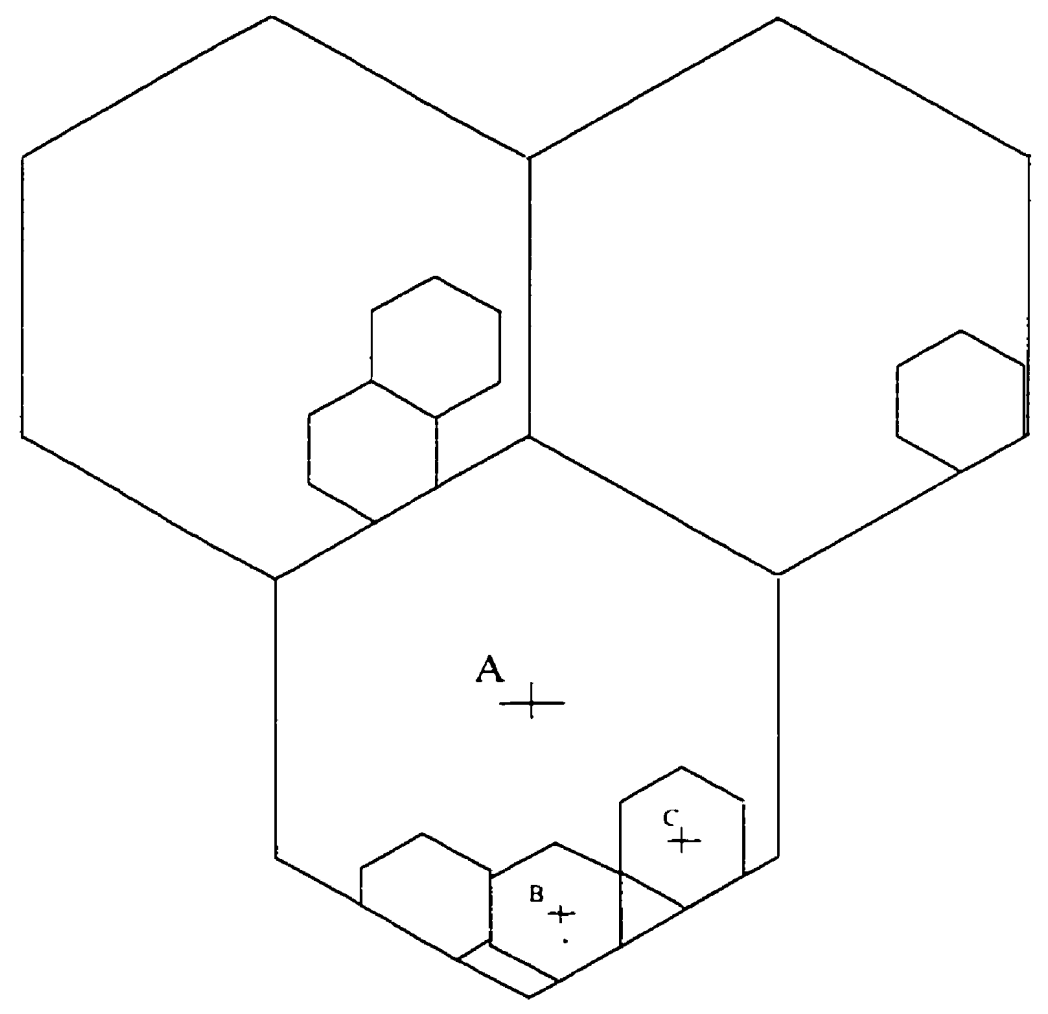

Figure 2.1: Overlaid Cellular Architecture 
The small cell operation has the disadvantage of large number of handoffs [37] when the mobile terminal moves rapidly through the coverage area. Thus larger cells are more appropriate from the point of view of handling fast moving terminals. However the large cell operation has an important implication on the cellular system's data handling capability. This is due to the relatively large channel delay spread. It is well known that maximum reliable transmission rate over a channel is inversely proportional to the channel delay spread. Over uniform terrain conditions delay spread is proportional to cell size i.e. small cell with shorter delay spread are capable of handling high rate transmission and vice versa. Thus as the cell size increases. the maximum transmission rate decreases and the use of channel equalizers become necessary if the large cell is to support higher data rates.

Recently a lot of researchers are investigating performance of multirate service in cellular environment. Some of the works are listed in bibliography section. 


\section{Chapter 3 \\ System Design and Description}

This chapter describes the details of the system used in simulation experiments. The transmitter and receiver architecture, channel model and spreading sequence used in the simulation are described. Several possible scenarios and SIR in a multirate CDMA cellular environment are also presented.

\subsection{Transmitter Model}

In a multiuser system, a number of users are transmitting simultaneously. The output of the $i$-th user is given by

$$
x_{i}=\sqrt{2} \operatorname{Re}\left\{\sum_{k=1}^{x} x_{i, k} c_{i}\left(t-k N T_{c}-\tau_{i}\right) e^{j\left(\left(\omega_{i} t+\phi_{1}\right)\right.}\right\}
$$

Each user has the same carrier frequency. $\omega_{c}=2 \pi f_{c}$. and $\phi_{i}$ is the phase angle of $i$-th user, $\tau_{i}$ is the delay for the $i$-th user and $T_{c}$ is the chip period. BPSK modulation is used for each user since BPSK gives performance similar to that of QPSK and is preferable as it uses one spreading code per user compared to two spreading codes per user needed for QPSK [24]. Each user encodes a binary data stream into BPSK $( \pm 1)$ code stream $\left\{x_{i .1}, x_{i .2}\right.$ $x_{i, 3} \ldots \ldots \ldots \ldots . . . .1$ The data symbol are assumed to be independent and identically distributed random variables, where $\operatorname{Pr}\left\{x_{i, k}=\mp 1\right\}=0.5$. The equivalent baseband equation is given by 


$$
x_{i}=\sum_{k=1}^{x} x_{i, k} c_{i}\left(t-k N T_{c}-\tau_{i}\right) e^{j \phi_{i}}
$$

Equation 3.2 is used for the present simulation. since it is convenient to use. $c_{i}(t)$, a $n$ chip code sequence assigned to the $i$-th user is defined as

$$
c_{i}(t)=\sum_{j=1}^{. v} c_{i j} \Psi\left(t-j T_{c}\right)
$$

where $c_{i j} \in\{ \pm 1\}$ are independent identically distributed random variables each occuring with a probability of 0.5 . It is assumed that each code spans exactly one symbol period i.e. the number of chips $N=T / T_{c}$ where $T$ is one symbol period. $\Psi(t)$ is chip pulse shape. For convenience it is assumed to be a rectangular pulse defined as

$$
\Psi(t)=\left\{\begin{array}{lr}
1 & \text { for } 0 \leq t \leq T_{c} \\
0 & \text { otherwise }
\end{array}\right.
$$

\subsection{Spreading Code}

Spreading codes used in simulation experiments are Gold code sequences containing - I s and I's. The Gold codes are generated by modulo- 2 addition of a pair of $m$-sequences. The code sequences are added chip-by-chip synchronously. The codes themselves are of the same length. Hence the two code generators maintain the same phase relationship. The codes generated have the same length as that of the two base codes. A typical sequence generator connected in Gold configuration $(N=3 l)$, used in the simulation experiment is shown in Figure 3.1. The autocorrelation and crosscorrelation of two Gold codes, for this (figure 3.1) specific Gold code configuration, is shown in Figure 3.2. 


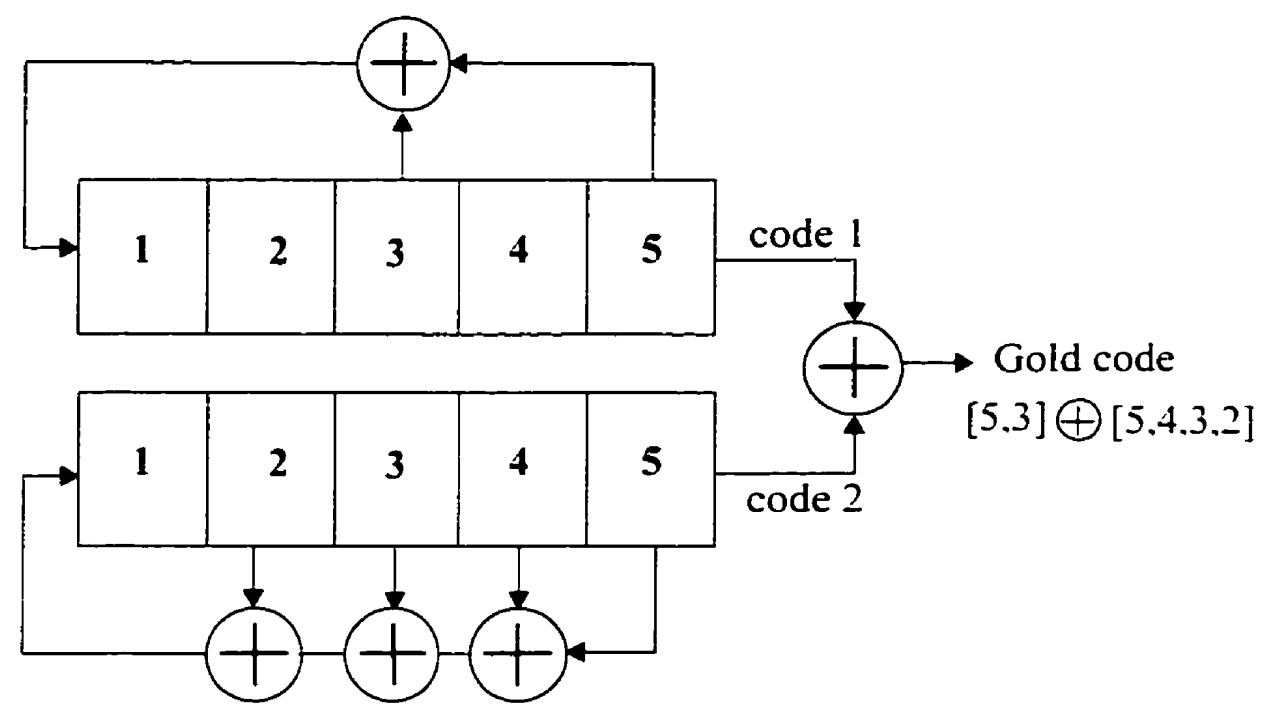

Figure 3.1: Illustration of Gold code generation

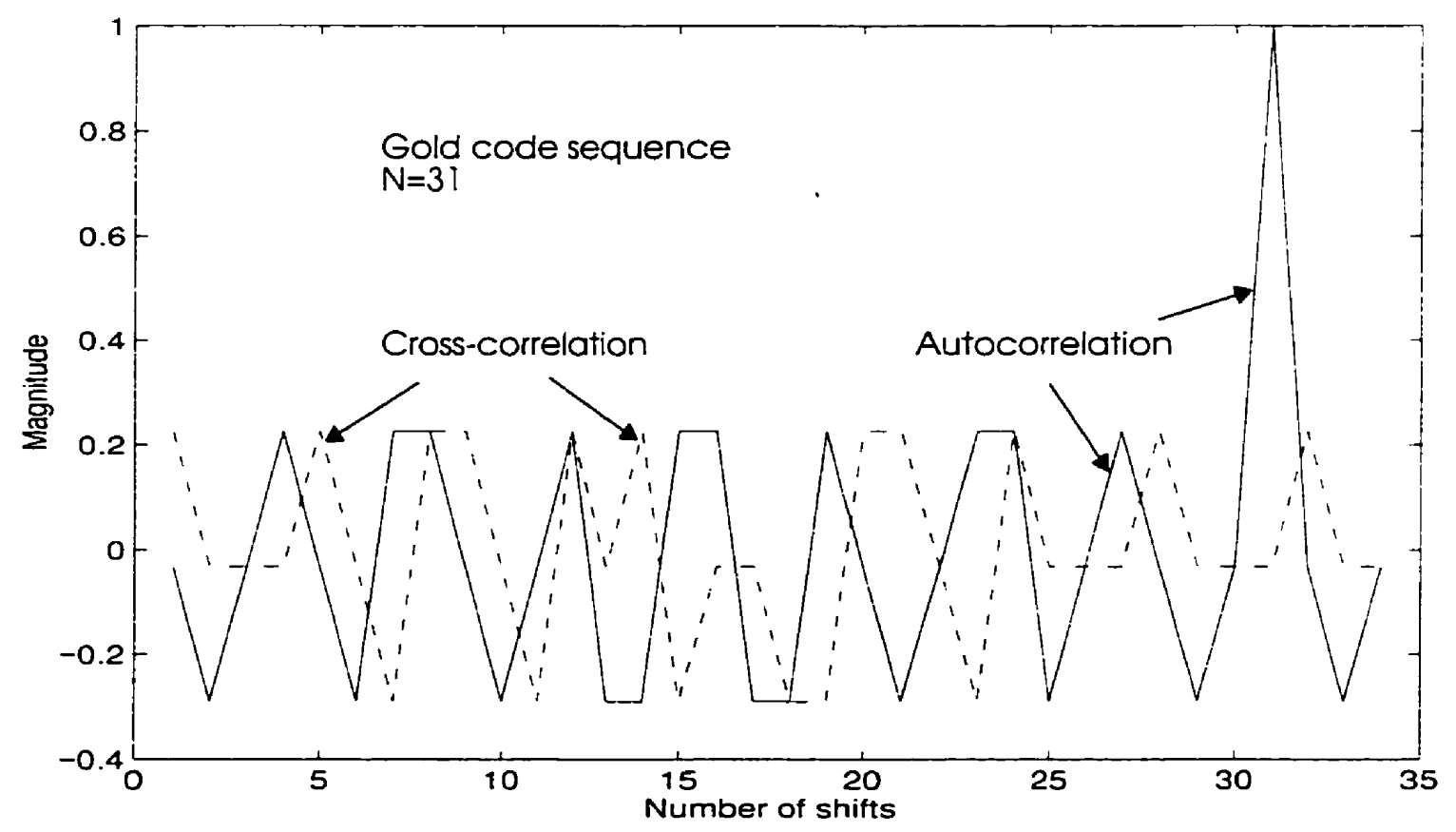

Figure 3.2: Normalized correlation of Gold code sequence 
Each user is assigned a unique sequence. The bit stream is then multiplied directly with the chip sequence, thereby giving the DS-SS system. It is assumed that bit stream and the chip sequence are clocked together in such a way that the number of chips in a bit interval is an integer, therefore the processing gain (PG) is equal to the code length $N$. To accommodate variable data rate in CDMA system following approaches are considered:

(i) Fixed transmission bandwidth (F_BW)

(ii) Variable transmission bandwidth (V_BW)

In F_BW scenario, all users occupy approximately same transmission bandwidth. To achieve different data rates, processing gain is varied accordingly, while keeping the chip rate $\left(R_{c}\right)$ fixed. In this case, simulation uses four samples per chip. The $P G$ 's selected for the simulation experiments are 15.31 and 63. As an illustrative example, if $R_{c}=1$ Mcps . then sampling rate. $R_{s}=4 M H z$. This give data rates of $R_{h l}=66.67 \mathrm{kbps} . R_{h_{2}}=32.25 \mathrm{kbps}$ and $R_{h 3}=15.87 \mathrm{kbps}$. for PG of 15,31 and 63 respectively, all having the same transmission BW of approximately $l M H=$.

On the other hand, in variable transmission bandwidth (V_BW) scenario. processing gain is kept fixed (which in this case is equal to 31 ). Here variable bit rate is achieved by varying the chip rate. That is. data at high rate is spread by high rate chip sequence and vice versa. For example, if $R_{c l}=1 \mathrm{Mcps}, R_{c 2}=500 \mathrm{kcps}$ and $R_{c 3}=250 \mathrm{kcps}$. i.e. $R_{c l}=2 R_{c_{2} 2}=$ $4 R_{c 3}$. then achievable data rates are $R_{b 1}=32 \mathrm{kbps}, R_{b 2}=16 \mathrm{kbps}$ and $R_{b 3}=8 \mathrm{kbps}$ respectively. This results in different transmission bandwidths, which are approximately $1 \mathrm{MHz}$, $500 \mathrm{kHz}$ and $250 \mathrm{kHz}$ respectively. These two cases are shown in Figure 3.3. 


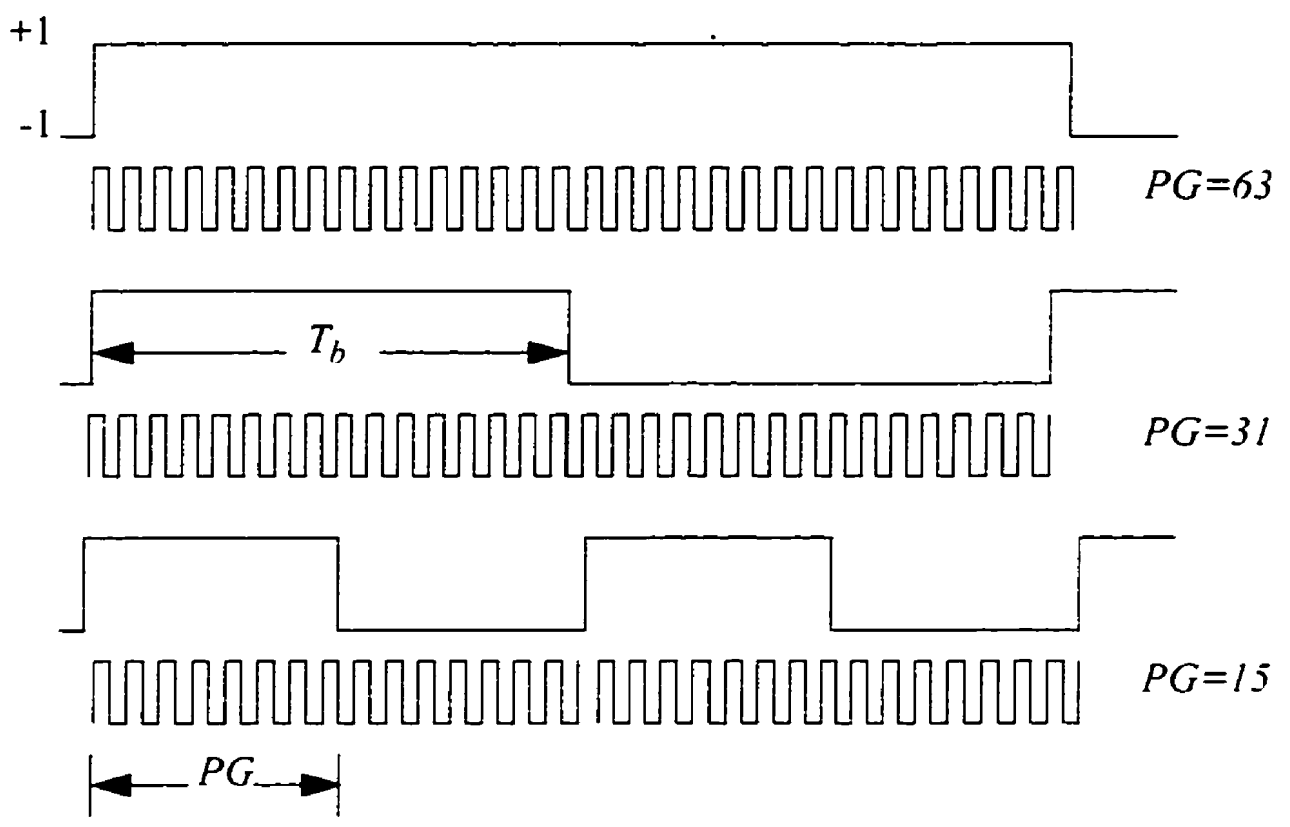

(a) Fixed bandwidth scenario (fixed chip period)
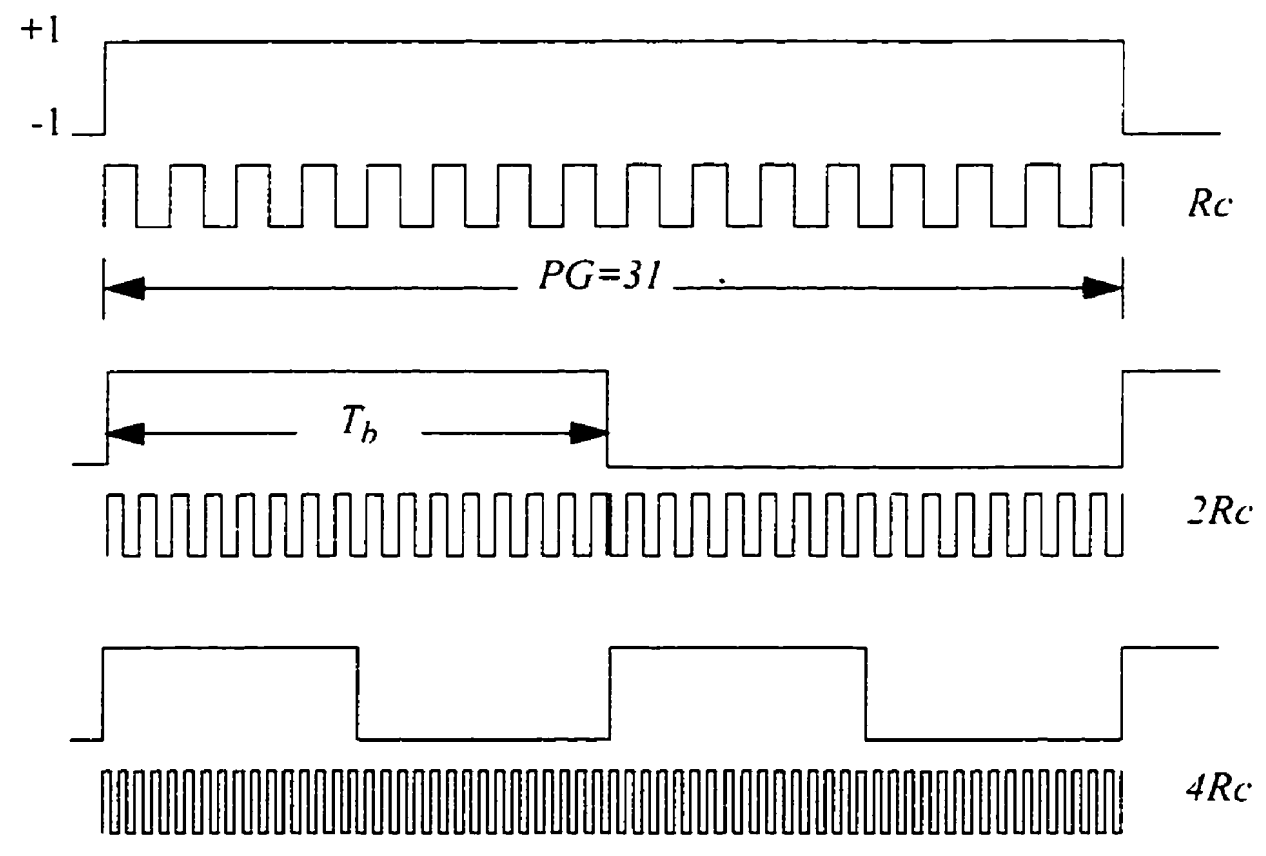

(b) Variable bandwidth scenario (variable chip period)

Figure 3.3: Data symbol and transmitted symbol 
In terms of overlaid cellular system. the delay spread in the microcell channel is less than that of larger cells because of lower transmitted power. As the maximum possible transmission rate is inversely proportional to delay spread, so a user in a microcell can support higher data rates than in a macrocell. Strict power control in CDMA system is very hard to maintain because of rapid changes in the mobile environment. Therefore, simulation experiments are carried out in an environment where the average power of the users are same.

\subsection{Channel Model}

The channel model used in this simulation is a WSSUS channel model. The channel has a fixed number of paths as shown in Figure 3.4 and is given by

$$
h(t)=\sum_{l=1}^{L} \alpha_{l}(t) e^{j \phi(l) t} \delta\left(t-\tau_{l}\right)
$$

where $\alpha_{l}(t)$ is Rayleigh distributed amplitude of the $l-t h$ path and $\phi_{l}(t)$ is the phase shift associated with that specific path. $L$ is the number of paths used and is equal to three for the simulation. $\tau_{l}$ is the delay associated with the $l-t h$ path. For the comparison purpose. the delay spread in the system is considered to be equal to six chip periods regardless of the chip rate. As the transmission bandwidth is much wider than the coherence bandwidth of the channel, the channel is frequency selective. As an illustrative example. we assume that the chip period. $T_{c}$, is equal to $1 \mu s$ (transmitted bandwidth is equal to $1 M H z$ ) i.e. maximum delay spread of $6 \mu \mathrm{s}$ is allowed. Therefore, the coherence bandwidth of the channel is $167 k H z(1 / 6 \mu s)$. This results in a data rate of approximately $32 k b p s$ at a processing gain of $31(\sim 15 d B)$. 
The channel consists of three paths randomly occuring within the first $6 T_{c}$ of a bit period. as shown in Figure 3.5. It is assumed that the first path of all the users occurs at the first chip so that all the users' transmissions are synchronized. The second and third paths occur randomly within the next five chip periods. It is possible for the second and third path to occur at the same chip period. But the simulation is designed in such a way that no more than a single path will occur at the same chip period. For instance, if the second path occurs at the second chip period, the third path will occur randomly within the next four chip periods. Again, if the random selection for the second path is fifth chip period, then the third path will occur in sixth chip period and so on. Each path consists of a complex Gaussian variable.

The variance of each path in the simulated channel model is assumed to decay exponentially according to the relation $e^{-f T, 3)}$, where $T_{A}$ is the arrival time of a ray relative to the first arrived ray. This is the so called exponential power delay profile [24]. [4]. The channel profile is normalized which means that the total variance of the three paths of the channel is equal to one. This also means that the sum of the instantaneous power of the three paths at a given time may not be equal to one but the sum of average over a long time will be equal to one. This type of channel model results due to an imprecise power control as the power of various users at the receiver will be equal in the average sense if the transmitted power is same. Although the average power of the users is same, the power of different users at a particular instant of time may be different. This type of power control is known as the average power control.

The noise added at the input of the receiver is additive and zero mean white Gaussian 


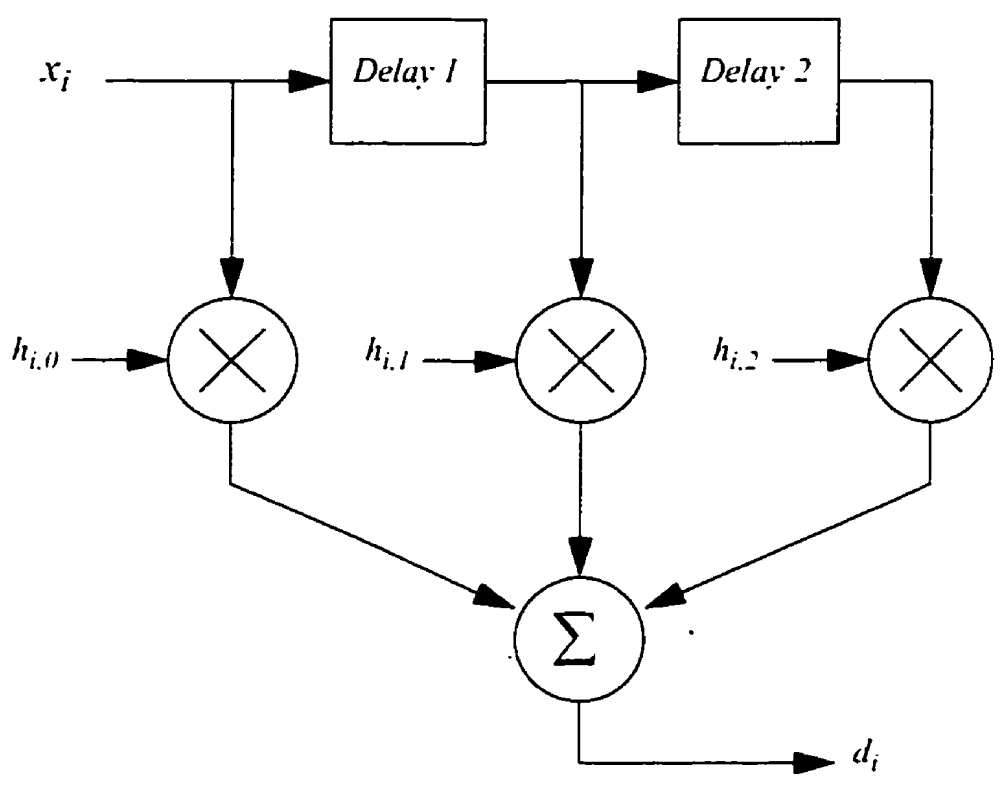

Figure 3.4: Three ray channel model

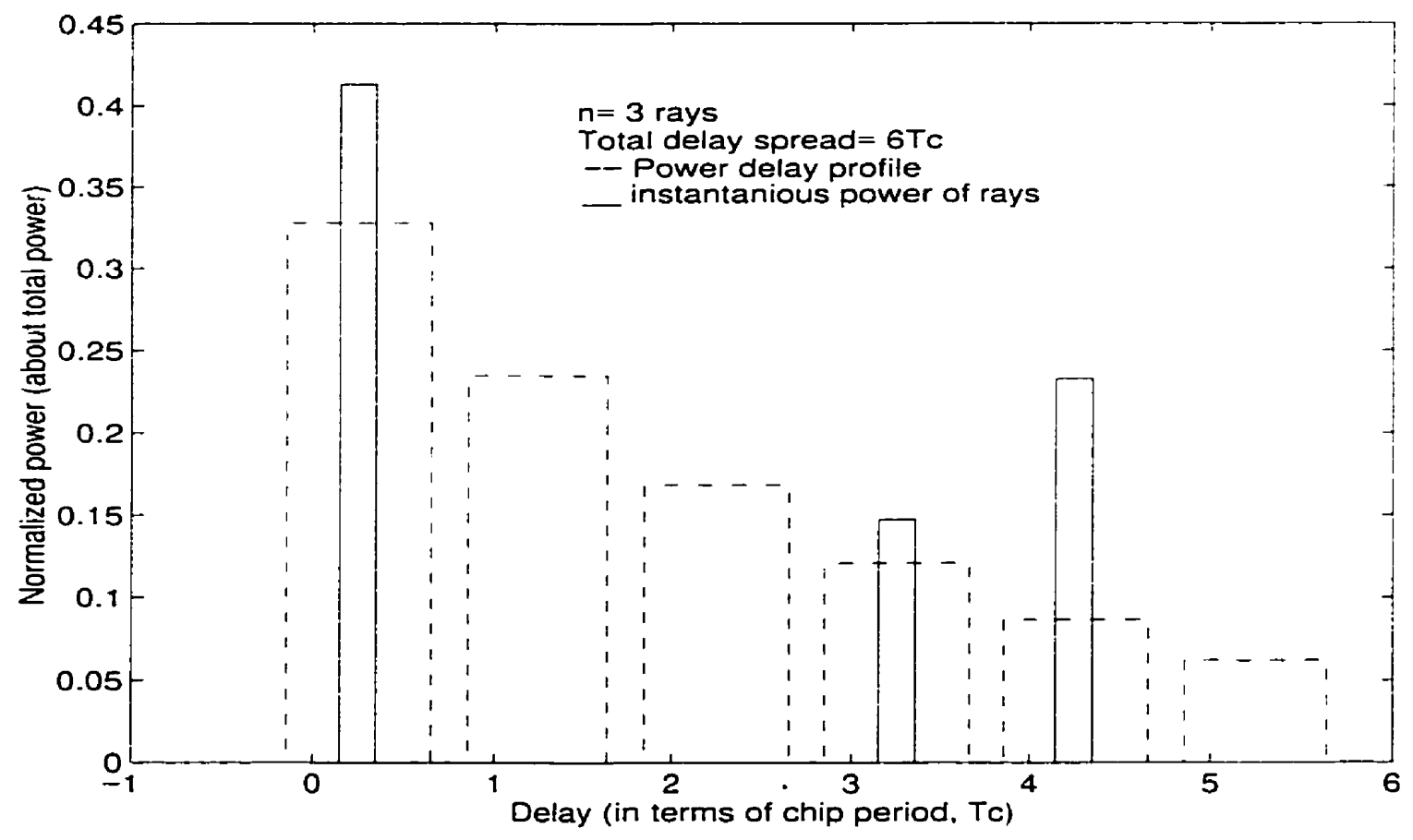

Figure 3.5: Exponentially decaying power delay profile 
(AWGN) with two sided power spectral density equal to $N_{\alpha} / 2$. The real and imaginary components of the noise are independent and uncorrelated. This is also true for the samples of noise as the autocorrelation of AWGN is a delta function. Effects of shadowing. typically modeled as log-normally distributed multiplicative interference. can be mitigated through the use of average power control [13]. Since the simulation experiments assume average power control, therefore, the effects of shadowing is not considered in the simulation. The signal after passing through the channel is given by

$$
d_{i}=\sum_{k=1}^{\infty} x_{i . k} \sum_{l=1}^{L} \alpha_{l}(t) e^{j \varphi_{l}(t)} c_{i}\left(t-k n T_{c}-\tau_{i}-\tau_{l}\right)
$$

The transmitter model considered in the system is shown Figure 3.6 along with the channel model and noise.

\subsection{Receiver Model}

The signal arriving at the receiver is given by

$$
d(t)=\sum_{i=1}^{K} d_{i}(t)+n(t)
$$

where $K$ is the number of users and $n(t)$ is AWGN. As the white noise process has two sided power spectral density of $\mathrm{No} / 2$ for all frequencies, the average noise power or noise variance is infinite. Therefore the received signal has been passed through a receiver filter to bandlimit the noise and to make the variance of the noise at the output of the receiver filter finite. The receiver filter used in the system consists of a filter which is matched to the spreading code and pulse shape of the desired user. The output of the matched filter is a function of its autocorrelation with the desired user and the crosscorrelation with the 


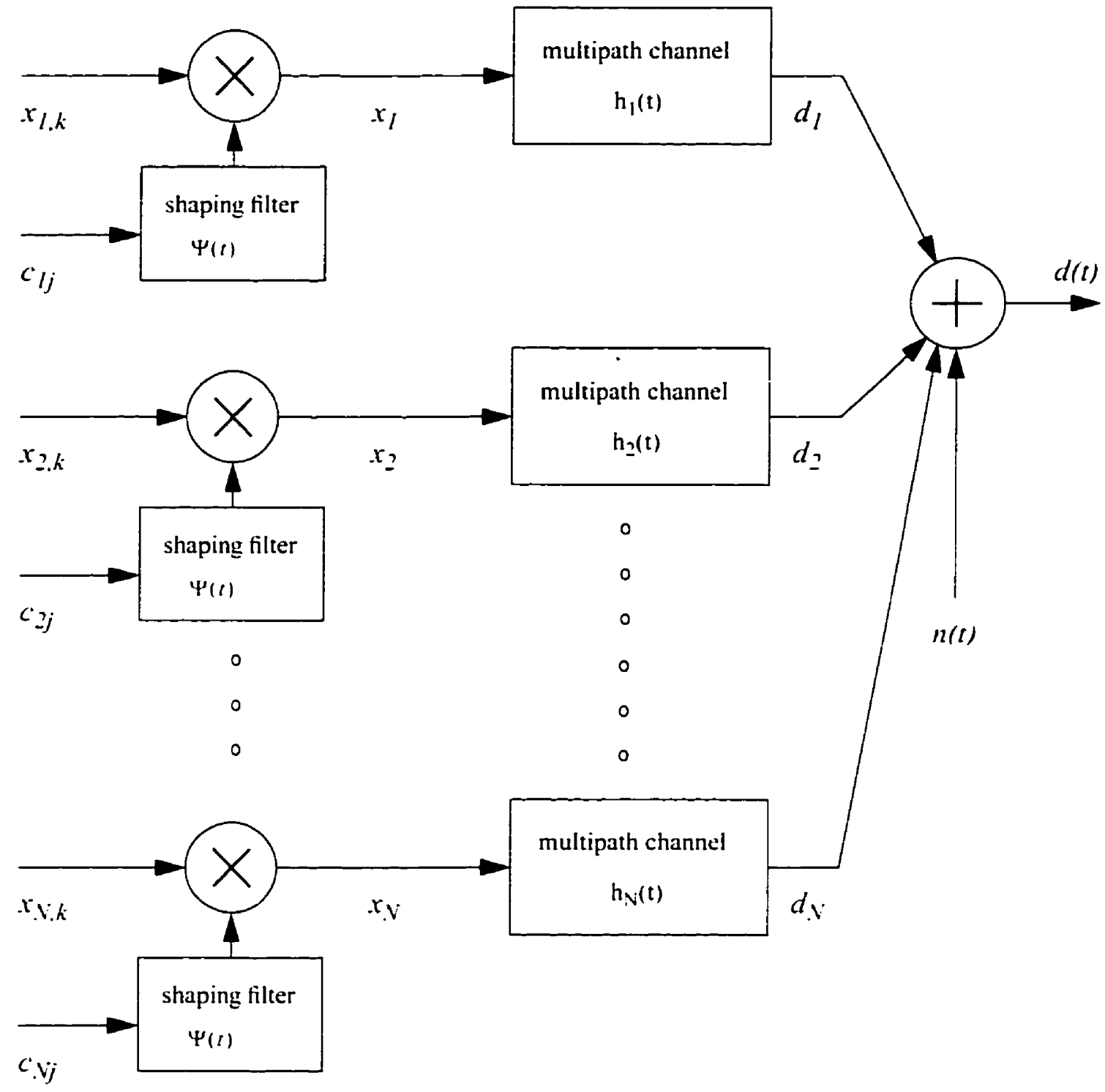

Figure 3.6: Transmitter and Channel model 
other users. As the cross correlation between the desired user and the other users is less than the autocorrelation function, this receiver filter (despreader) helps in reducing the effect of interfering users on the desired user. The output of the filter is sampled at the chip rate before being fed to the forward filter of the decision feedback equalizer.

RLS algorithm is used to update the taps of the DFE. The main advantage offered by the RLS algorithm is its fast convergence rate which is necessary to track the rapid changes taking place in an outdoor environment. The RLS algorithm is relatively insensitive to variations in the eigenvalue spread of the correlation matrix of the tap input vector which is a problem with the Least Mean Square (LMS) algorithm. The LMS algorithm has much slower convergence rate than that of the RLS algorithm. The RLS algorithm is, however. computationaly complex compared to the LMS algorithm- a trade off to achieve fast convergence. The receiver structure is shown in Figure 3.7.

The output of the receiver filter is denoted by $v(t)$ while $v_{i}$ denotes the sample taken at time $i T_{c}$. These chip spaced samples constitute the input to the feedforward filter while the feedback filter gets output of the decision device at every symbol. The tap input vector is

$$
\overline{i_{k}}=\left[\begin{array}{lllllll}
v_{i+1} & v_{i+f-1} & v_{i+l-2 \ldots \ldots \ldots} & v_{i+1} & v_{i} & \dot{x}_{1, k-2} & \bar{x}_{1, k-h}
\end{array}\right]^{T}
$$

where $y_{k}$ represents the tap input vector needed to make decision for the $k$-th symbol. The feedback filter taps have the past decisions corresponding to the first user at their inputs as user- $I$ is assumed to be the desired user. The number of feedforward tap is $(f+I)$ and feedback tap is ' $b$ ' giving a total of $M=f+b+l$ taps. The input to the decision device is given by

$$
x_{1, k}^{\cdot}=\bar{y}_{k}^{T} \bar{w}_{k-1}
$$

where $\boldsymbol{w}_{k-l}$ is the column vector of equalizer coefficients updated at time $(k-l)$ and used at 
time $k$. The vector $\boldsymbol{w}_{k-1}$ is given by

$$
\bar{w}_{k-1}=\left[\begin{array}{lllll}
w_{k-1}(-f) & w_{k-1}(-f+1) \ldots & w_{k-1}(0) & w_{k-1}(1) \ldots & w_{k-1}(b)
\end{array}\right]^{T}
$$

A decay factor $\lambda$ is used which has a value slightly less than one. This weighting factor helps to forget the past data which is suitable for a time varying channel characteristics. The equalizer coefficient vector is determined so as to minimize the time averaged weighted square error. The following set of equations summarize the algorithm used to update the coefficients and have been used in the simulation

$$
\begin{gathered}
e_{k}=\bar{x}_{1, k}-\hat{x}_{1, k} \\
k_{k}=p_{k} v_{k}^{*}=\frac{p_{k-1} v_{k}^{*}}{\lambda+y_{k}^{T} p_{k-1} v_{k}^{*}} \\
p_{k}=\frac{1}{\lambda}\left[p_{k-1}-k_{k} v_{k}^{T} p_{k-1}\right] \\
w_{k}=u_{k-1}+k_{k} e_{k}
\end{gathered}
$$

$e_{k}$ is the equalizer output error at time $\mathrm{k}, p_{k}=R^{k-l}$ is the inverse of the correlation matrix $R_{k}{ }^{l}$. Since it is computationaly complex to calculate the inverse matrix in each iteration. the matrix $p_{k}$ is computed recursively using the above equations. It is necessary that an initial value is set for $p_{k}$. This value is set at

$$
p_{0}=\delta^{-1} I
$$

where $I$ is a $(M x M)$ identity matrix, the value of $\delta$ is selected to ensure nonsingularity of $R_{k}$. The $(M x l)$ order matrix $k_{k}$ is called the Kalman gain vector. The fast convergence speed of the RLS algorithm is due to the fact that each tap is controlled by one element of 


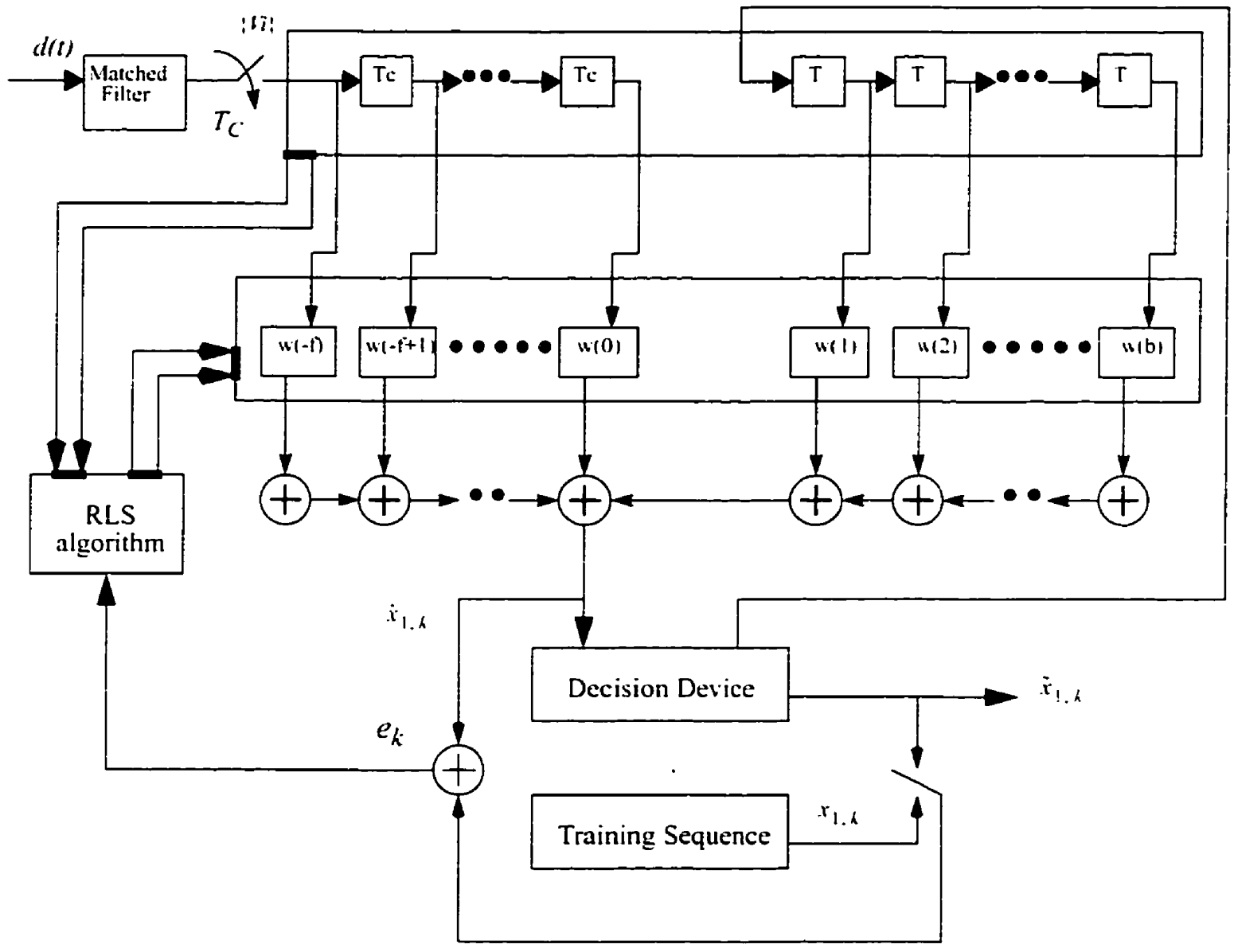

Figure 3.7: Receiver Structure 
$k_{k}$ which is adjusted at every iteration. Therefore, the RLS algorithm has $M$ adjustable parameters compared to only one adjustable parameter, the step size, available in LMS algorithm. It is customary to set the initial value of equalizer coefficient vector equal to a null vector. Therefore, $w_{0}=0$.

\subsection{Simulation Scenarios}

A number of different scenarios emerge in a multirate cellular structure as the mobile station moves around. A Rician channel will result when the desired user has a line of sight with the base station. The possibility of having a Rician channel is low compared to having a Rayleigh faded channel in the present context. Rayleigh faded channel has worse effect on the performance than the Rician channel. Therefore. in order to obtain the relative worst case scenario, Rayleigh faded channel is considered throughout the model. Three different data rates are considered for voice. low data (L-data) and high data (Hdata) where voice has the lowest bit rate and $\mathrm{H}$-data has the highest bit rate. It is also considered that in V_BW case, the information rates are integer multiple of each other. But this is not the case for the F_BW scenario. This is to reduce complexity of the system.

A different number of scenarios can occur. For example, the desired user may be transmitting L-data while the interferers are transmitting $\mathrm{H}$-data and voice and so on. Among various combinations of information rates within desired and interfering users, scenarios shown in Table 3.1 are considered for the simulation. All the scenarios are simulated and investigated in this thesis. Initially simulations of.Table 3.1 are executed for equal average power for all users. Then the same scenarios are considered keeping $E_{b}$ same for all users 
regardless of their bit rate. Finally simulations for some selected scenarios are performed in imperfect power control case where the average power of interferers differ $\mp 5 d B$ relative to the average power of the desired user.

Table 3.1: Simulation Scenario for Variable bandwidth case

\begin{tabular}{|c|c|c|c|c|c|}
\hline \multirow{2}{*}{$\begin{array}{l}\text { Desired users' } \\
\text { Information }\end{array}$} & \multirow{2}{*}{$\begin{array}{c}\text { Scenario } \\
\#\end{array}$} & \multicolumn{3}{|c|}{ Number of Interfering users } & \multirow{2}{*}{$\begin{array}{l}\text { Total user } \\
\text { (desired+int } \\
\text { erfere) }\end{array}$} \\
\hline & & H-data & L-data & Voice & \\
\hline \multirow{6}{*}{$\begin{array}{c}\text { H-data } \\
\mathrm{R}_{\mathrm{b}}=32 \mathrm{kbps}\end{array}$} & \multirow[t]{4}{*}{1} & 4 & 5 & 5 & 15 \\
\hline & & 2 & 2 & 3 & 8 \\
\hline & & 1 & 2 & 2 & 6 \\
\hline & & 1 & I & 1 & 4 \\
\hline & \multirow[t]{2}{*}{2} & 1 & 9 & 4 & 15 \\
\hline & & 1 & 4 & 2 & 8 \\
\hline \multirow{6}{*}{$\begin{array}{c}\text { L-data } \\
\mathrm{R}_{\mathrm{b}}=16 \mathrm{kbps}\end{array}$} & \multirow[t]{4}{*}{3} & 5 & 4 & 5 & 15 \\
\hline & & 3 & 1 & 3 & 8 \\
\hline & & 2 & 1 & 2 & 6 \\
\hline & & 1 & 1 & 1 & 4 \\
\hline & \multirow[t]{2}{*}{4} & 2 & 8 & 4 & 15 \\
\hline & & 2 & 3 & 2 & 8 \\
\hline \multirow{6}{*}{$\begin{array}{c}\text { Voice } \\
\mathrm{R}_{\mathrm{b}}=8 \mathrm{kbps}\end{array}$} & \multirow[t]{4}{*}{5} & 5 & 5 & 4 & 15 \\
\hline & & 3 & 2 & 2 & 8 \\
\hline & & 2 & 2 & 1 & 6 \\
\hline & & 1 & 1 & 1 & 4 \\
\hline & \multirow[t]{2}{*}{6} & 2 & 9 & 3 & 15 \\
\hline & & 2 & 4 & 1 & 8 \\
\hline
\end{tabular}


Simulation is also performed to investigate the effect of combination of interferers on performance. For example. the only difference between scenarios $I$ and 2 is the combination of interferers. The same difference exists between scenario 3 and 4 and scenario 5 and 6 . The scenarios in Table 3.1 are simulated for variable bandwidth case because the aim of the research is to investigate the performance under variable chip rate scenario. For the purpose of comparison with fixed bandwidth case. scenarios in Table 3.2 are simulated and compared with that of Table 3.1

Table 3.2: Simulation Scenario for Fixed bandwidth case

\begin{tabular}{|c|c|c|c|c|}
\hline Total user & $P G=15$ & $P G=31$ & $P G=63$ & Desired \\
\hline 15 & 5 & 5 & 5 & \multirow{4}{*}{$P G=15$} \\
\hline 8 & 3 & 2 & 3 & \\
\hline 6 & 2 & 2 & 2 & \\
\hline 4 & 2 & 1 & I & \\
\hline 15 & 5 & 5 & 5 & \multirow{4}{*}{$P G=63$} \\
\hline 8 & 3 & 2 & 3 & \\
\hline 6 & 2 & 2 & 2 & \\
\hline 4 & 1 & 2 & 1 & \\
\hline
\end{tabular}

\subsection{SIR in multiple data rate scenario}

For a single cell site with power control, all reverse link signals are received at the same power level. It is stated in [22] that for $K$ users, each cell site demodulator processes a composite received waveform containing the desired signal having power $P$ and $(K-1)$ interfering signals each having power $P$. In this case, the interference is modeled as white 
Guassian noise. Thus the signal to interference power ratio (before despreading) is

$$
S I R=\frac{P}{(K-1) P}=\frac{1}{(K-1)}
$$

Here thermal noise has not been considered since CDMA system is more susceptible to interference than thermal noise. In multiple data rate scenario, each user or each group of users are transmitting data at different rates and hence their $P$ or $E_{h}$ or both will be independent and vary accordingly from others. Assuming $k_{l}$ users are transmitting at rate $R_{l}$. $k_{2}$ users are transmitting at rate $R_{2} \ldots k_{m}$ users are transmitting at rate $R_{m}$, where $K=k_{1}+k_{2}+\ldots+k_{m}$. It is also assumed that $R_{l}>R_{2}>\ldots R_{m}$. where $m$ is the number of groups each containing $k_{x}$ users. Since interference has linear effect on the performance of the CDMA system [22], therefore assuming large $K$ in multiple data rate scenario. equation (3.16) can be written in generalized form as

$$
S I R=\frac{P_{1}}{k_{1} P_{1}+k_{2} P_{2}+\ldots+k_{m} P_{m}}
$$

where desired user is chosen from group $k_{l}$ having power $P_{l}$. This scenario is shown in Figure 3.8 for three groups each transmitting at different data rate and assuming the data rates to be integer multiple of each other. For the sake of convenience, assuming $R_{I}=2 R_{2}$ $=4 R_{3}$, the following two cases are considered.

(i) case-I: This case is referred to as equal power case, where all users power is same. i.e. $P_{l}=P_{2}=\ldots \ldots \ldots . .=P_{m}$. Therefore, as shown in Figure 3.8(a). energy per bit $\left(E_{h}\right)$ of different data rate users will vary according to their $R_{b}$. Taking H-data rate $\left(R_{l}\right)$ user as the desired user, equation 3.17 can be written in terms of bit energy to noise density ratio as 

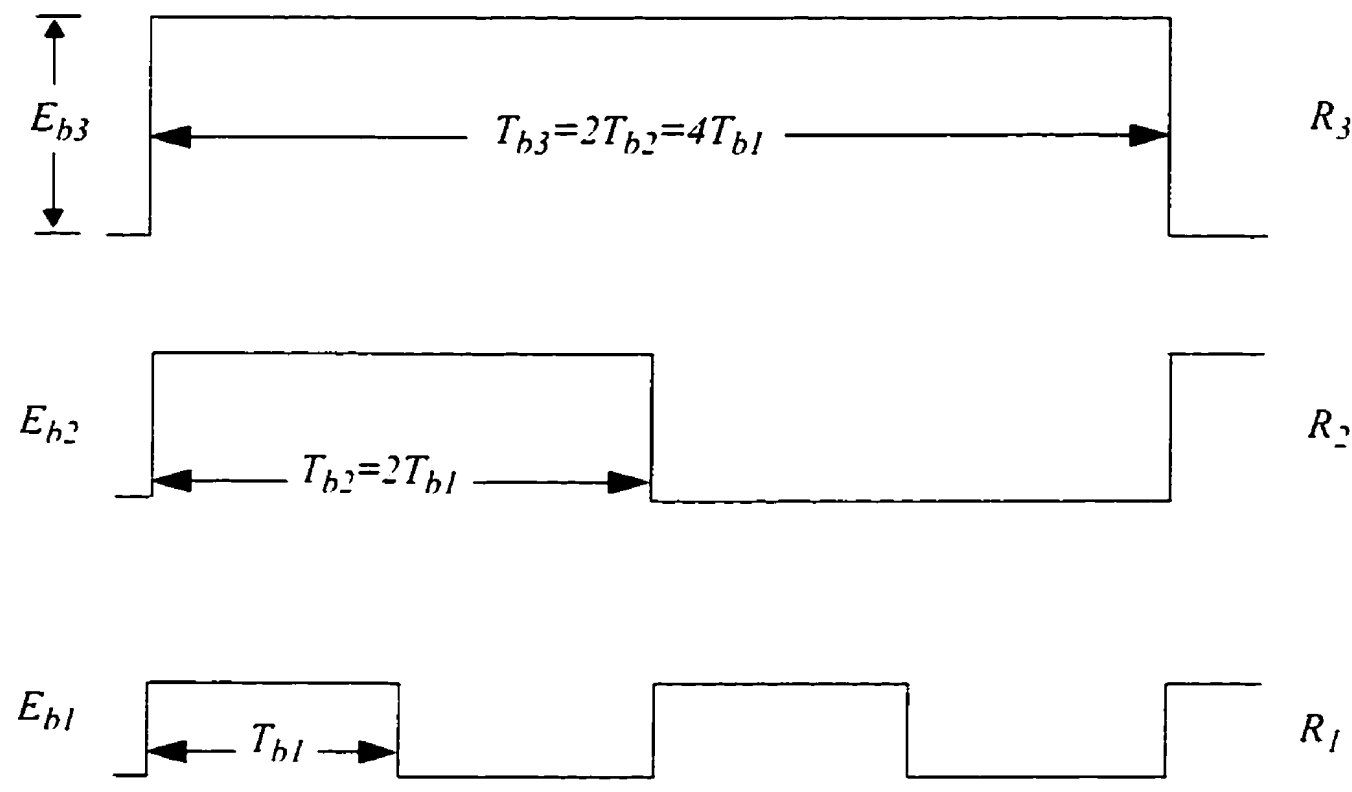

(a) Same Power $\left(P=E_{b} / T_{b}\right)$ for all users

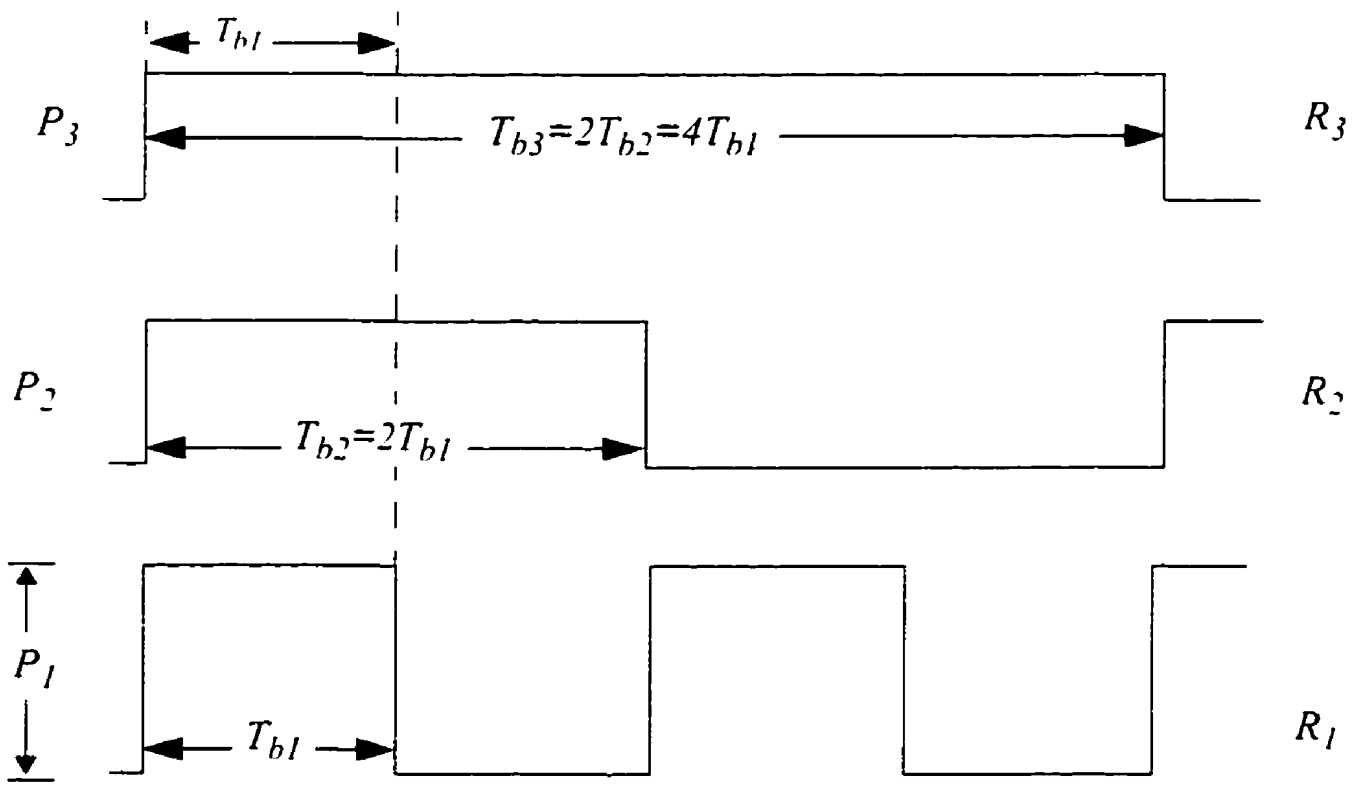

(b) Same Bit Energy $\left(E_{b}=P_{m} T_{b}\right)$ for all users

Figure 3.8: Power and Energy relationship in multiple data rate 


$$
\frac{E_{h 1}}{N_{0}}=\frac{P / R_{1}}{\left[\left(k_{1} P\right) / R_{1}+\left(k_{2} P\right) / R_{2}+\left(k_{3} P\right) / R_{3}\right] / W}
$$

where the term in square bracket is the noise (or interference) and $W$ is the total bandwidth. Substituting parameters obtained from Figure 3.8(a) into equation (3.18) yields.

$$
\frac{E_{h 1}}{N_{0}}=\frac{W / R_{1}}{k_{1} / R_{1}+k_{2} / 0.5 R_{1}+k_{3} / 0.25 R_{1}}=\frac{W}{k_{1}+2 k_{2}+4 k_{3}}
$$

On the other hand, if the desired user is chosen from the moderate data rate $\left(R_{2}\right)$ users. then equation (3.19) has the form

$$
\frac{E_{b 2}}{N_{0}}=\frac{W / R_{2}}{k_{1} / 2 R_{2}+k_{2} / R_{2}+k_{3} / 0.5 R_{2}}=\frac{W}{0.5 k_{1}+k_{2}+2 k_{3}}
$$

Similarly, if desired user is from lowest data rate $\left(R_{3}\right)$ users. then equation $(3.19)$ has the form

$$
\frac{E_{h 3}}{N_{0}}=\frac{W / R_{3}}{k_{1} / 4 R_{3}+k_{2} / 2 R_{3}+k_{3} / R_{3}}=\frac{W}{0.25 k_{1}+0.5 k_{2}+k_{3}}
$$

Comparing equation (3.17) through (3.21), it can be seen that at same transmission power. low data rate users are more immune to bit errors than high data rate users (although all types of users experience same SIR). At the same time number of lower data rate users dominate the overall performance, i.e. reduced number of lower data rate user will improve the overall performance. As a special case, when $W$ is fixed and $k_{l}=k_{2}=k_{3}$, then $E_{b 3} / N_{0}$ is 4 times higher than $E_{b l} / N_{O}$. Therefore, at the same power level. performance is not only limited by SIR, but also depends on the desired users bit energy and the combination of interferers as shown in equation (3.19) to (3.21).

(ii) case-II: In this case, referred to as the equal energy case, it is assumed that all users' energy per bit $\left(E_{h}\right)$ is same regardless of their data rate, i.e. $E_{b l}=E_{b 2}=\ldots \ldots \ldots . .=E_{b m}$. 
Therefore, power of different data users will vary according to their $R_{b}$, as shown in Figure 3.8 (b). When the desired user is chosen from highest data rate $\left(R_{l}\right)$ user, equation 3.17 can be written as

$$
S I R=\frac{P_{1}}{k_{1} P_{1}+k_{2} P_{2}+k_{3} P_{3}}
$$

where $P_{1}, P_{2}$ and $P_{3}$ are the power of respective data users. Replacing parameters from Figure 3.8(b) into equation (3.22) for highest data rate user yields.

$$
S I R=\frac{P_{1}}{k_{1} P_{1}+0.5 k_{2} P_{2}+0.25 k_{3} P_{3}}=\frac{1}{k_{1}+0.5 k_{2}+0.25 k_{3}}
$$

When desired user is from moderate data rate $\left(R_{2}\right)$ users, then equation (3.23) has the form

$$
S I R=\frac{P_{2}}{2 k_{1} P_{2}+k_{2} P_{2}+0.5 k_{3} P_{2}}=\frac{1}{2 k_{1}+k_{2}+0.5 k_{3}}
$$

In the same way, when the desired user is from lowest data rate $\left(R_{3}\right)$ users. then equation (3.23) has the form

$$
S I R=\frac{P_{3}}{4 k_{1} P_{3}+2 k_{2} P_{3}+k_{3} P_{3}}=\frac{1}{4 k_{1}+2 k_{2}+k_{3}}
$$

Comparison of equation (3.23) to (3.25) reveals that even if $E_{h}$ is same for all users but signal power has influence on the performance. This is seen by comparing bit energy over interval $T_{h l}$. Here H-data rate user has $E_{b l}=P_{l} T_{b l}$ and sees interference coming from Ldata and voice rate users. The interferers have bit energy $E_{h 2}^{\prime}=P_{2} T_{h 1}$ and $E_{h 3}^{\prime}=P_{3} T_{h 1}$ respectively over period $T_{h l}$. Since $P_{l}=2 P_{2}=4 P_{3}$, hence, $E_{h l}=2 E_{h 2}^{\prime}=$ $4 E_{h 3}^{\prime}$. Therefore, high data rate users have advantage over low data rate users. This is just the reverse scenario of case-I. Therefore, at same $E_{h}$. performance is dictated by desired 
users signal power and the combination of interferers as shown in equation (3.23) through (3.25).

Case-I and case-II can be applied for F_BW case in a straight forward manner. In V_BW case. as in the F_BW case. the low bit rate users have the advantage in the equal power case. This is because the entire signal spectrum of the voice rate user is masked by the signal spectrum of other users. Hence the interference experienced by voice rate users can be considered as white Gaussian. But this may not be true for H-data and L-data rate users. For $\mathrm{H}$-data rate (L-data) users, interference is non-white due to voice and L-data rate (voice) users. Therefore, the relative performances in the equal power and equal $E_{h}$ cases remains to be seen. Simulation experiments carried out to investigate the performance in these cases, are described in the next chapter. 


\section{Chapter 4}

\section{Results}

The results of the simulation experiments are presented in this chapter. Suitable values of receiver parameters are investigated e.g. effect of the feedforward and feedback filter length on the performance of the receiver. Scenarios tabulated in Table 3.1 are chosen for the simulation and results of each scenario are presented. In the final section the effect of imperfect power control on the performance is considered. The performance is evaluated in terms of BER. MSE and probabilities of outage determined by MSE and BER are presented.

\subsection{Receiver Parameters Initialization}

Before discussing the simulation results, several suitable parameters such as length of feedforward and feedback taps for the receiver need to be determined. The feedforward filter of the DFE is used to cancel the precursor ISI while the feedback filter is used to cancel the postcursor ISI.

For RLS algorithm the computational complexity is proportional to the square of the number of taps. Therefore, it is necessary to adjust the equalizer length properly. The system consists of users transmitting at different data rates. For service integration, however it is desirable to determine an equalizer length which is the same for all users no matter what the data rate and what approach is applied (F_BW or V_BW). In simulation experiments. $E_{b} / N_{\emptyset}$ is varied from $6 d B$ to $30 d B$ for all users, where $E_{b}$ is defined as the bit energy and 
$N_{0}$, the two sided noise power spectral density. The value of chip energy to noise power spectral density is then given by $E_{c} / N_{\Omega}(d B)=E_{b} / N_{\Omega}(d B)-10 \log P G$. The objective of this research is to investigate the performance due to multiple access interference at several signal to noise ratios.

In order to determine the optimum length of the forward filter, a single user system is simulated for the following cases:

(i) desired user at $\mathrm{H}$-data rate ( $32 \mathrm{kbps}$ )

(ii) desired user at L-data rate ( $16 \mathrm{kbps})$

(iii) desired user at voice rate $(8 \mathrm{kbps})$

The simulation uses the above data rates, the delay profile shown in Figure 3.4. 3.5 and the transmitter structure in 3.6. The data transmitted by the user is multiplied by the spreading code and after passing through a three path fading channel, arrives at the receiver. To determine the length of the filter, each path is assumed to undergo independent Rayleigh fading. The received signal is passed through the receiver filter matched to the spreading code of the desired user and the chip spaced samples are fed to the DFE. In order to determine the feedforward filter length. the DFE is simulated with two feedback filter taps which is sufficient as found by earlier experiments [57]. [58]. The receiver in this case is trained using a training sequence of 40 symbols. It is assumed that the receiver has an accurate carrier recovery. This assumption reduces the simulation complexity. simulation time and memory required in the workstation.

The results of the simulation are shown in Figure 4.1 and Figure 4.2. In both figures the mean square error, in decibels, at the output of the equalizer is plotted as a function of the 


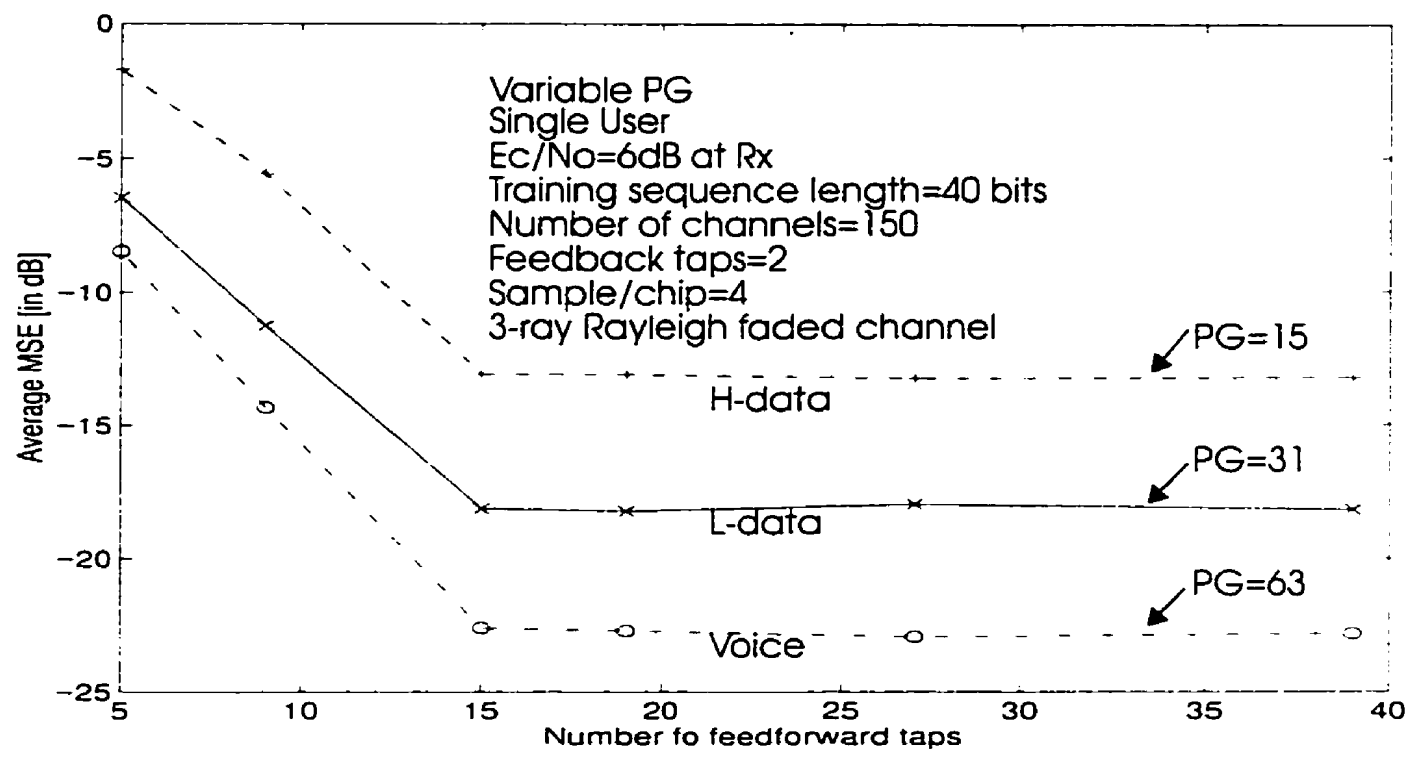

Figure 4.1: DFE performance as a function of number of feedforward taps for a single user in F_BW case

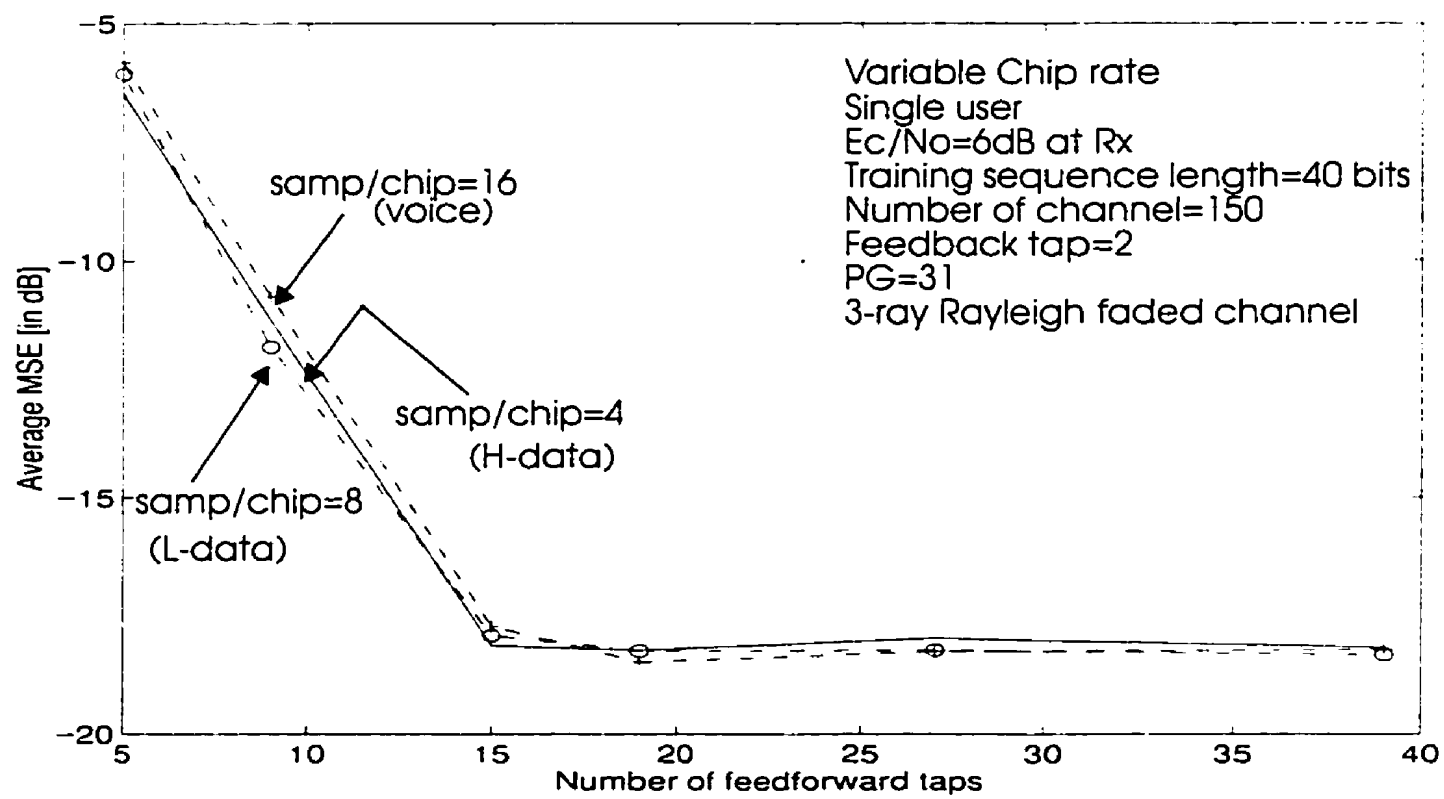

Figure 4.2: DFE performance as a function of number of feedforward taps for a single user in $\mathbf{V} \_\mathbf{B W}$ case 
number of the feedforward filter taps. It can be seen from these plots that no improvement from these plots is obtained if the number of taps is increased beyond 15. The reason is that the feedforward filter tries to cancel the precursor ISI for which it need to have a length longer than the delay spread of the channel. So the length of the feedforward filter is determined by the channel delay spread which in our case is $6 T_{C}$.

In the absence of multiple access interference, a length of 15 tap is therefore reasonable choice for the feedforward filter. But in multiuser environment a longer forward filter helps to reduce the effect of interference. Hence, it may be advantageous to have a longer feedforward filter. However, increasing the filter length beyond a certain limit may result in reduction in performance as the tap coefficients add to the noise at the output. A longer filter length also adds to the computational complexity of the tap coefficient update algorithm. Therefore to determine suitable forward filter length, additional experiments in the presence of interference were conducted.

At this stage. desired user data rate is set at $\mathrm{H}$-data (32kbps) while the interferers are from a combination of users of different data rates ( 2 from $\mathrm{H}$-data, 2 from L-data and 3 from voice). Experiment is conducted for a feedforward tap length of 15.19.27,39 and 71 taps keeping feedback taps equal to two. It is found that feedforward tap length beyond 27 does not show any significant improvement in performance compared to the complexity. Therefore a length of 27 taps will be used for the feedforward filter in all the simulations unless otherwise specified.

After fixing the length of feedforward filter to 27 taps the performance of DFE is simulated for feedback filter length of 1,2 and 3 taps. It is found that the mse $(d B)$ is equal to 


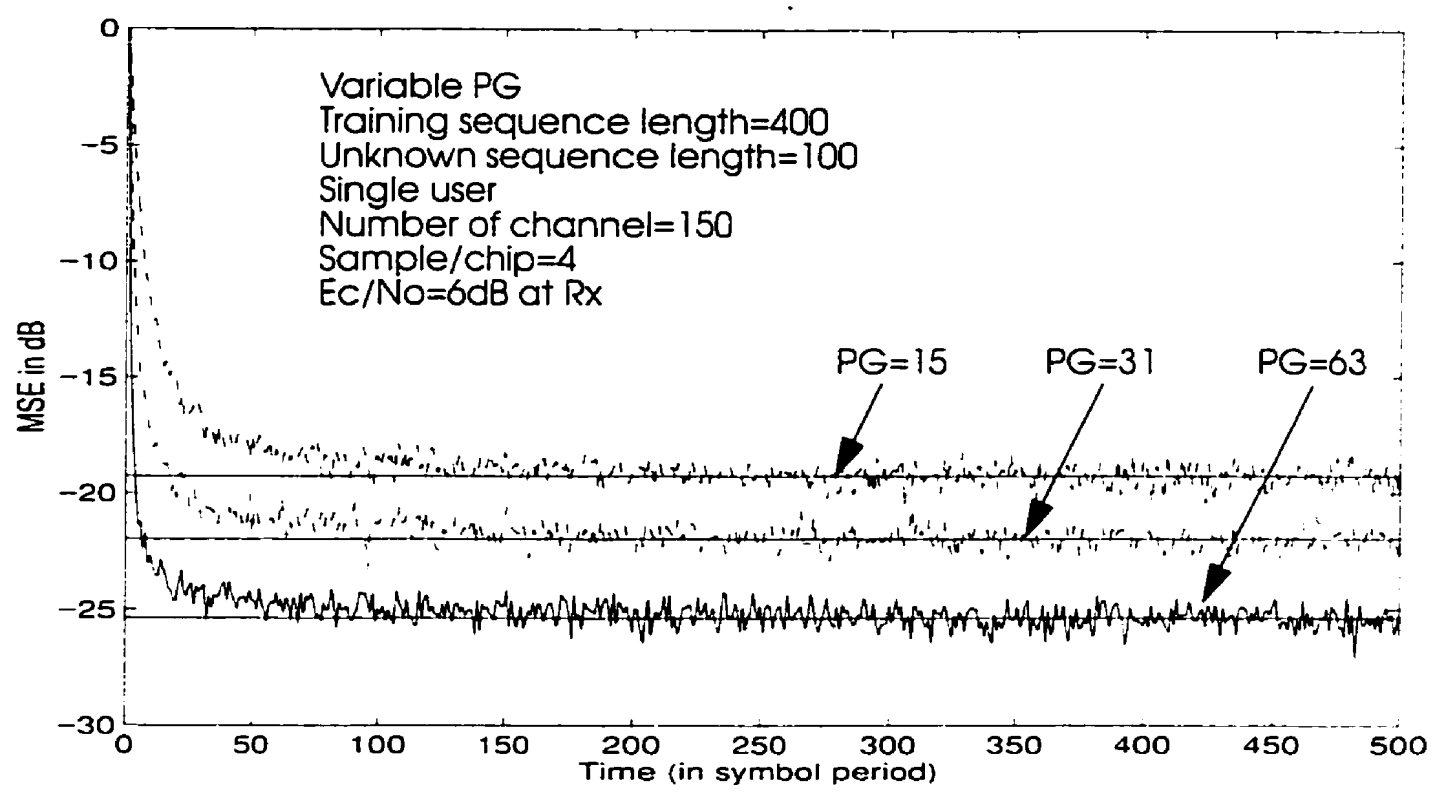

Figure 4.3: Convergence of $\operatorname{RLS}$ DFE $(27,2)$ receiver in $\mathbf{F} \_B W$

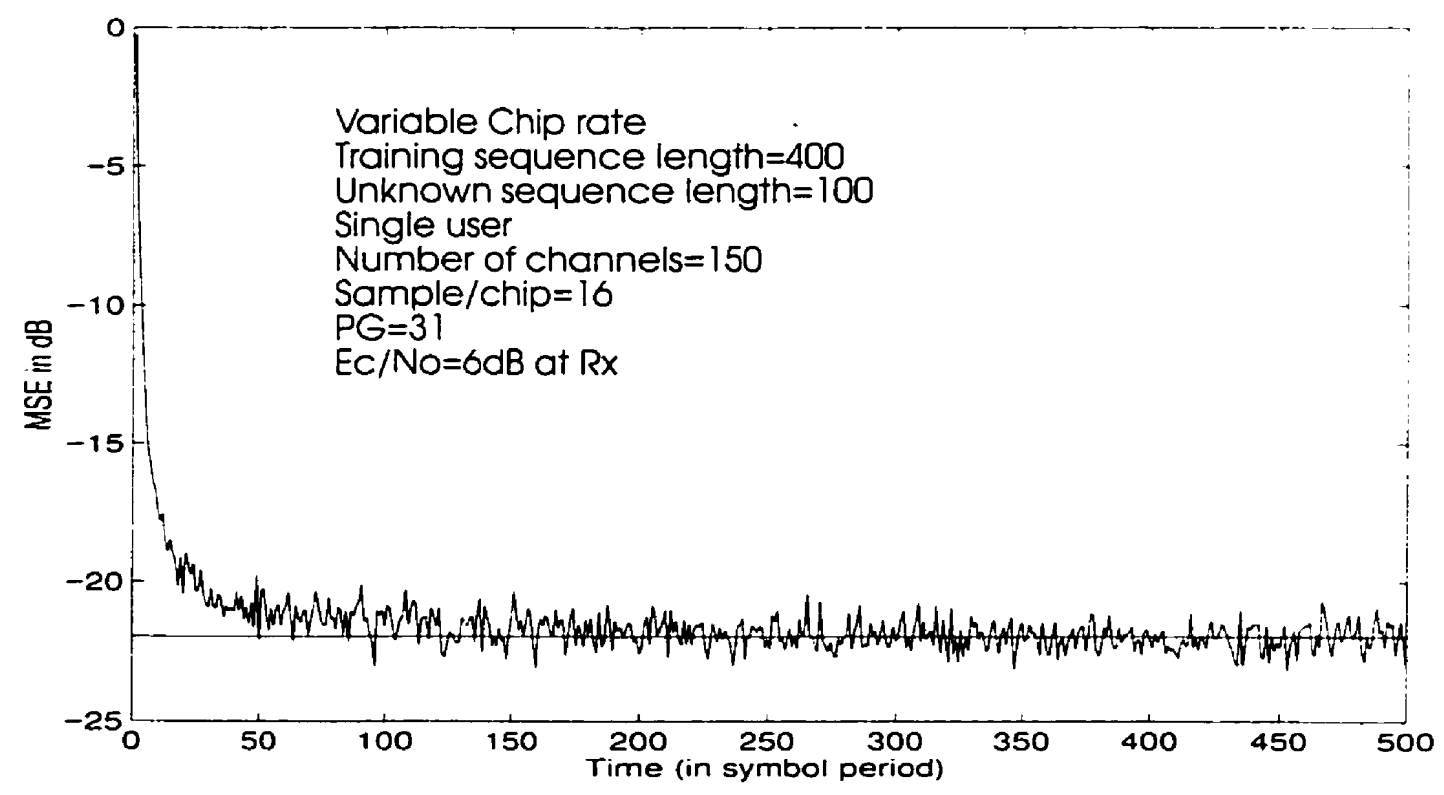

Figure 4.4: Convergence of $\operatorname{RLS}$ DFE $(27,2)$ receiver in $\mathbf{V} \_B W$ 
the values shown in Figure 4.1 and 4.2. The reason is that the ISI is mostly due to the neighboring symbols, so a feedback filter length of one tap is sufficient. However. a length of two feedback taps will be employed in all the following simulations for the same reason as discussed for the feedforward tap. The value of $\lambda=0.95$ is used in all the simulations. The convergence of RLS DFE $(27.2)$ receiver using the parameters discussed above is shown in Figure 4.3 and 4.4. Here the simulation is done for 400 training bits and 100 unknown bits. It is seen that the algorithm converges around 50 symbols. This confirms the finding of [57] that the RLS algorithm converges in the mean square sense in a number of symbol periods equal to twice the number of equalizer taps. As the length of equalizer filter used in simulation is 29 taps, therefore a training sequence of 60 symbols is appropriate and this length is used throughout the simulation.

\subsection{Calibration of the System}

The probability of bit error for binary phase modulation in AWGN channel can be expressed as [63]

$$
P_{e}=Q\left(\sqrt{\frac{2 E_{h}}{N_{0}}}\right)
$$

The calibration of the system starts with simulation performed under AWGN channel. BPSK modulated data is multiplied by the spreading code before transmitting over the channel: this arrives at the receiver via a single path containing AWGN. At the receiving end data is received using $\operatorname{RLS} \operatorname{DFE}(27,2)$ receiver as shown in Figure 3.6. The AWGN channel is then replaced by a three ray exponential power delay profile Rayleigh faded channel as shown in Figure 3.4 and the same receiver is used to receive data. The result of simulations are shown in Figure 4.5. It is seen that in AWGN channel the simulated result 
matches expression (4.1). On the other hand, in Rayleigh faded channel the system performance degrades beyond $E_{b} / N_{0}=15 \mathrm{~dB}$. This is due to rapid and unpredictable changes of the channel parameter and inability of the DFE receiver to deal with increased ISI. Figure 4.5 also shows the operating region of the system. To improve reliability, the simulation experiments were repeated five times. The output of these simulation runs are shown in Figure 4.6. From this figure it is seen that ail simulation runs give close results.

The probability of error in Rayleigh faded channel (no multipath) in the presence of interference (F_BW case), can be expressed as (detail mathematics is shown in appendix A)

$$
P_{e}=\frac{1}{2} \int_{0}^{x} \frac{(K-1) x^{K-2}}{(x+1)^{K}} \operatorname{erfc}\left(\sqrt{\frac{3 N}{2 x}}\right) d x
$$

where $K$ is the number of users and $N$ is length of spreading code. Here it is assumed that the average power of the desired user and the interferers are same implying an interference limited system where noise may be neglected. A simulation is executed for high SNR $(40 d B)$ in a single ray Rayleigh faded channel for a number of users. All users spread with spreading gain of $3 \mathrm{I}$ and the desired user transmit at a rate of $32 \mathrm{kpps}$ ( $\mathrm{H}$-data). The output of simulation and analytical result using expression (4.2) is shown in Figure 4.7. The simulated result matches closely to the theoretical one for large number of users but not for 6 of lesser number of users.

This is because of the Gaussian approximation used to model the interference in expression (4.2). The use of Gaussian approximation to determine the average probability of error in expression (4.2) for binary, CDMA. multiple access communication systems is based on the argument that the decision statistic, $Z_{0}=I_{0}+n+\zeta$, may be modeled as a 


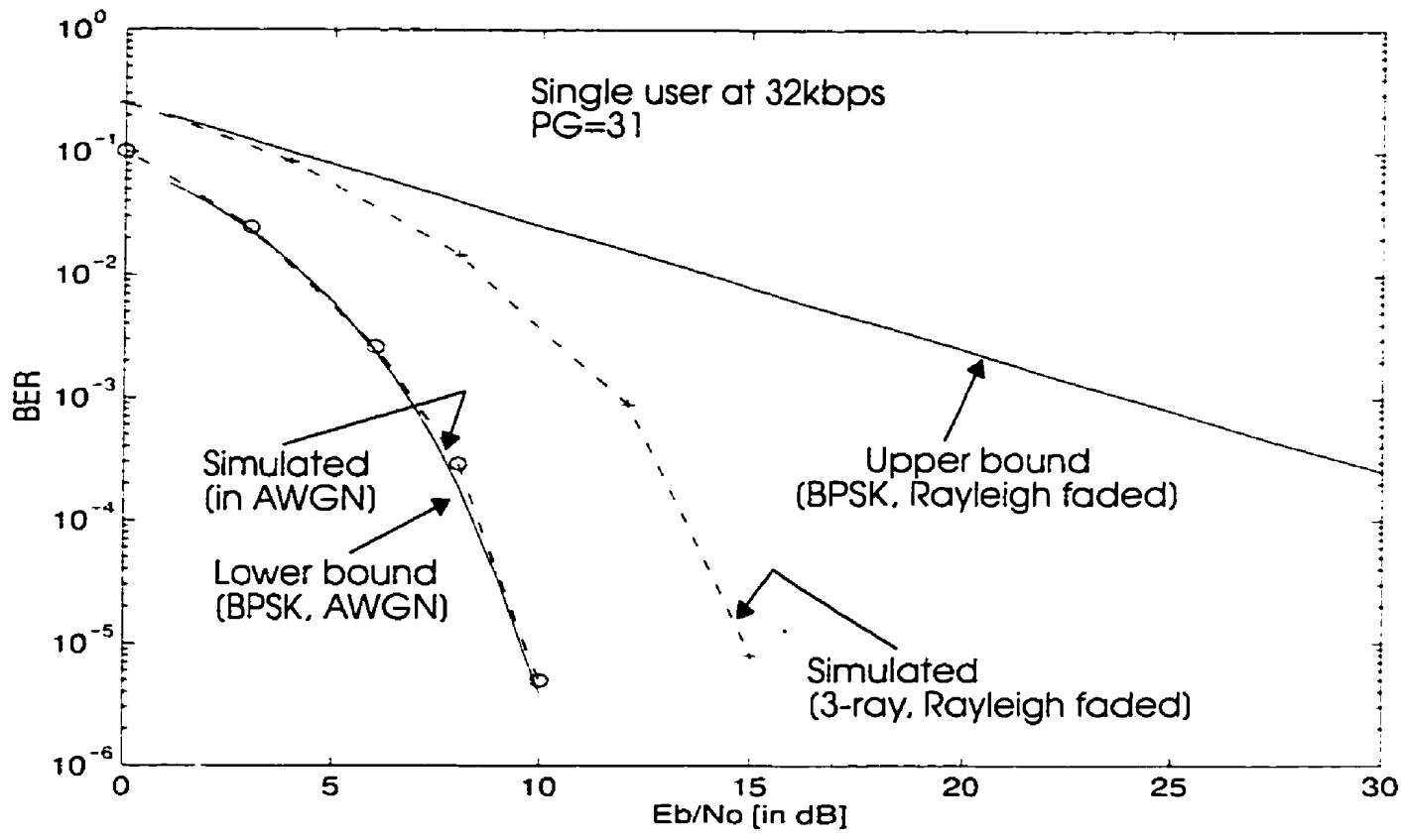

Figure 4.5: System performance in single user case

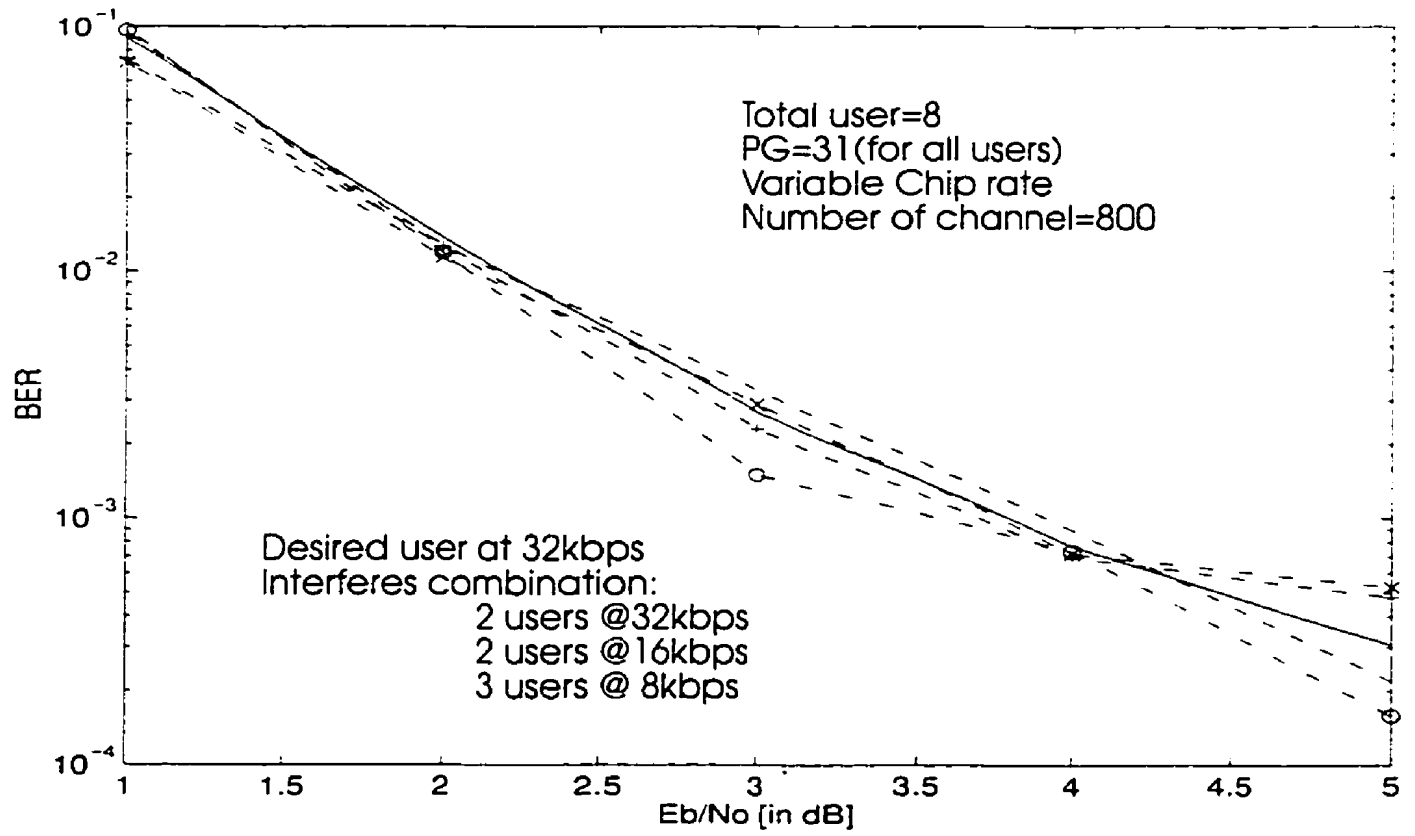

Figure 4.6: System stability in multiuser case (V_BW) 


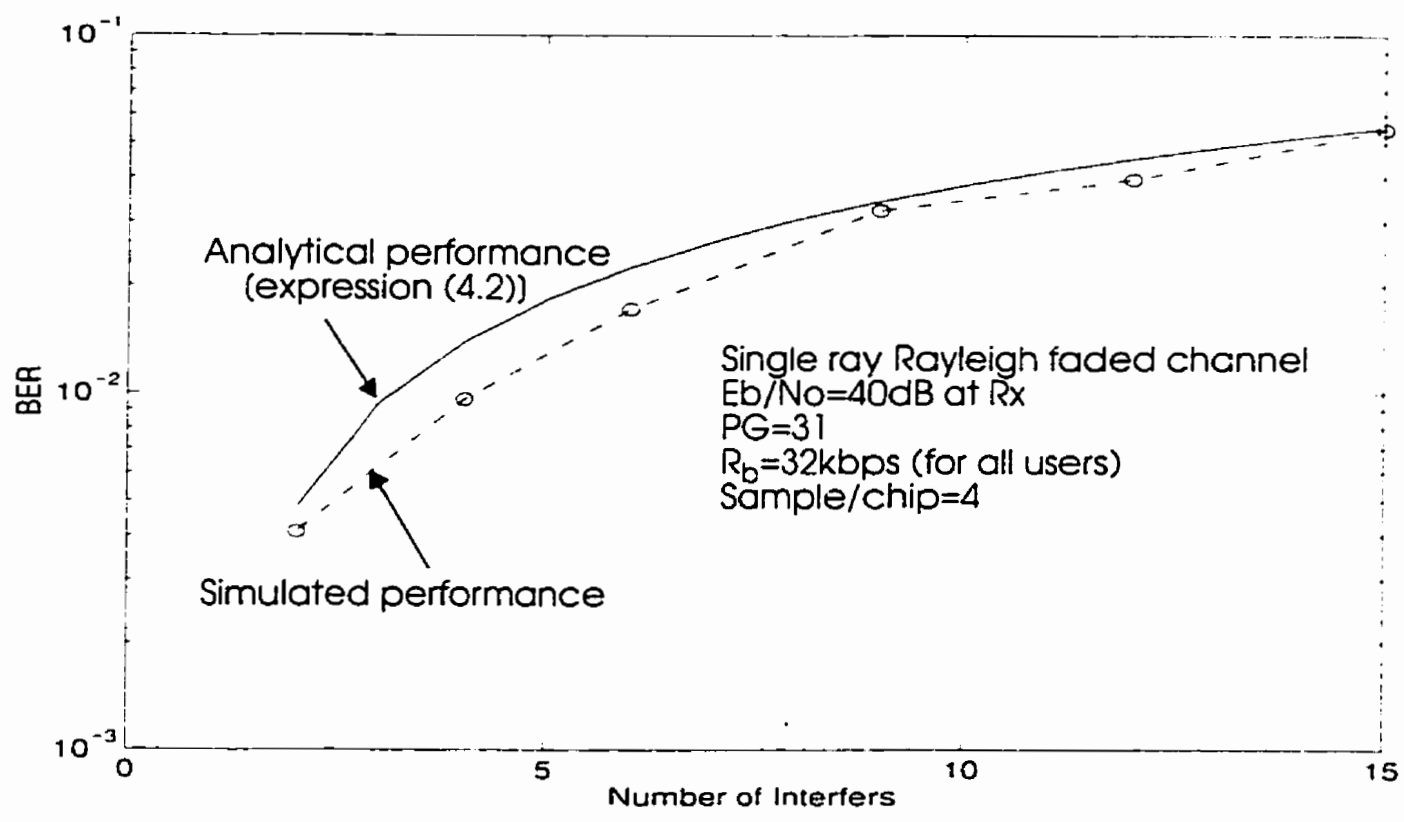

Figure 4.7: System performance in multiuser case $\left(F_{-} B W\right)$ 
Gaussian variable [48]. Here $I_{0}$ is the desired contribution to the decision statistic from the desired user and is deterministic. The noise contribution. $n$, and multiple access interference from co-channel users, $\zeta$, are assumed to be zero mean Gaussian random variables. According to CLT, the random variable $\zeta$, is approximately normally distributed as the number of interferers become large. As suggested in [60], the Gaussian approximations are valid when the number of users, $K$. is large. However, when the number of interferers are small, the Gaussian model is not accurate. This argument is reflected on Figure 4.7, for number of users lesser than 6 .

\subsection{Results for $V \_B W$ case}

In chapter 3. several possible scenarios in the proposed overlaid system were discussed. Significant difference between the scenarios is the result of different information rate. channels and number of interferers. As discussed in section 3.2. simulation result for each cases (F_BW and V_BW) will be presented but V_BW case will remain our focus. Each of the scenario presented in Table 3.1 will be discussed in detail for V_BW case only. In order to obtain the plots, the system is simulated for 150 to 2000 randomly generated individual channels for each user while $E_{h} / N_{0}$ is varied from $6 d B$ to $30 \mathrm{~d} B$. Each simulation run lasts for 500 symbols and is preceded by a training sequence of 60 symbols. The MSE and outage are plotted at $E_{h} / N_{o}=18 \mathrm{~dB}$ at the transmitter end. 


\subsubsection{Equal Average Power for each user}

\subsubsection{Desired user at $\mathrm{H}-$ data rate}

This scenario presents the case in which the desired user is transmitting at $32 k b p s$ and interference is coming from a combination of users as shown in Table 3.1. The plots for this case are shown in Figure 4.8,4.9,4.10 and 4.1 I. From Figure 4.8. it is seen that as the number of users increases, the BER performance degrades which is expected. As seen from Figure 4.8, for a total user of 6,8 and 15. BER converges towards a irreducible error rate, but it is not that significant for a total user of 4 . This is because of high interference, which is the performance limiting factor in the mobile environment. It is also seen that up to $18 \mathrm{~dB}$. BER performance for 8 users is very close to that of 6 users. however. 6 user case performs better beyond $18 d B$. It is due to the effect of interference (section 3.6) that has been imposed by the user combination (table 3.1) for these two cases. Figure 4.9 shows the similar results for these two cases as well as the performance degradation with increased number of users. The minimum MSE at output of the receiver is given by $\operatorname{MMSE}(d B)=-\mathrm{SNR}(d B)$ where a SNR of $6 d B$ is considered satisfactory for the performance of a coded coherent system [57]. This can be seen from Figure 4.9 that the value of MSE is much less than $-6 d B$ when $E_{h} / N_{O}$ is $18 d B$ at the transmitting end.

It should be noted that DFE performs the function of Rake by combining the energies in the multipaths while cancelling the cochannel interference and ISI. This advantage of DFE over Rake is shown in [57] for same data rate users and in [58] for different data rate users. It can also be observed in Figure 4.9 that the value of MSE $(d B)$ does not vary by more than $3 d B$ after 60 symbols which form the training sequence. So the length of training sequence used in the simulation is adequate. 


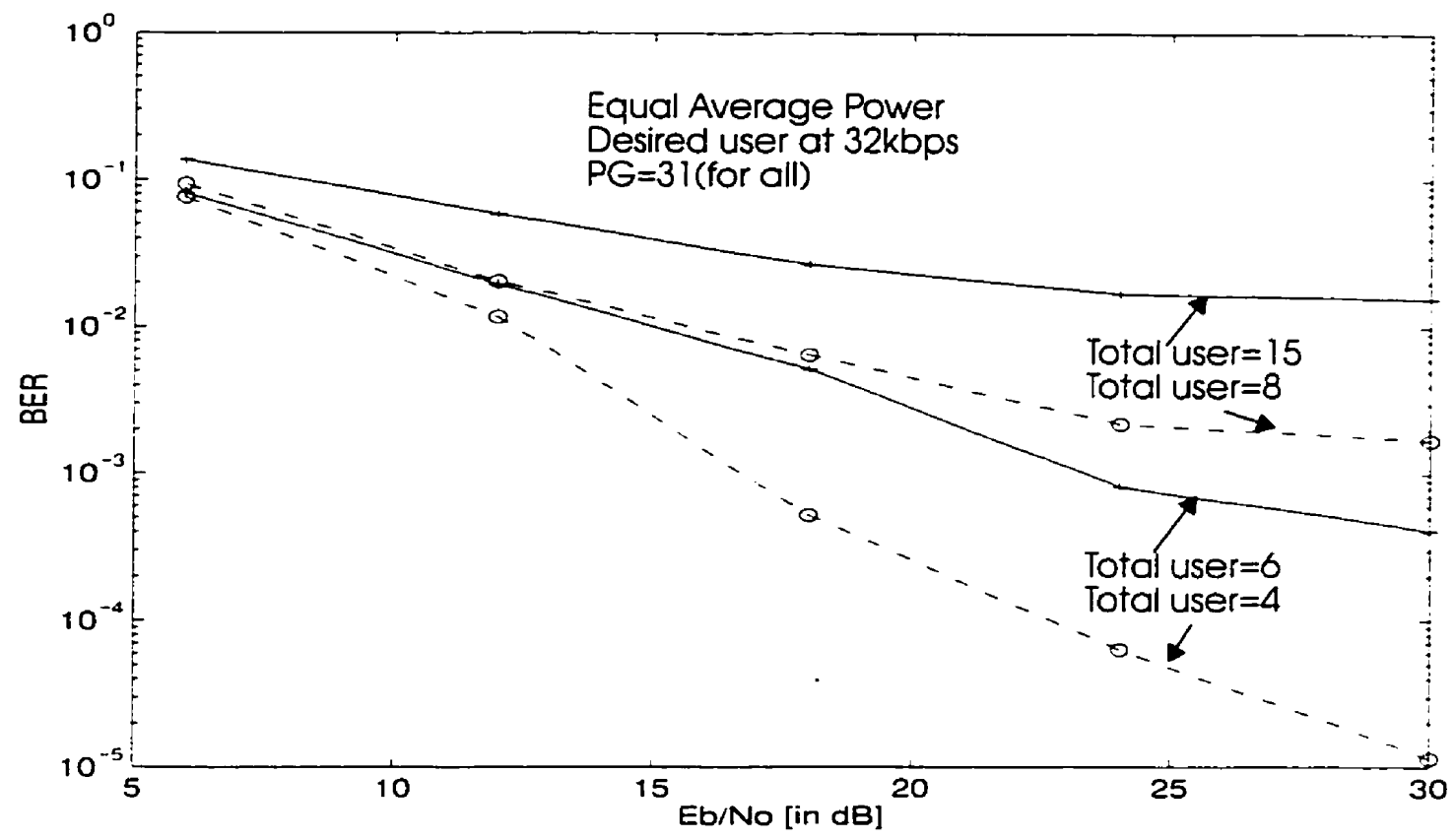

Figure 4.8: BER Performance in scenario 1

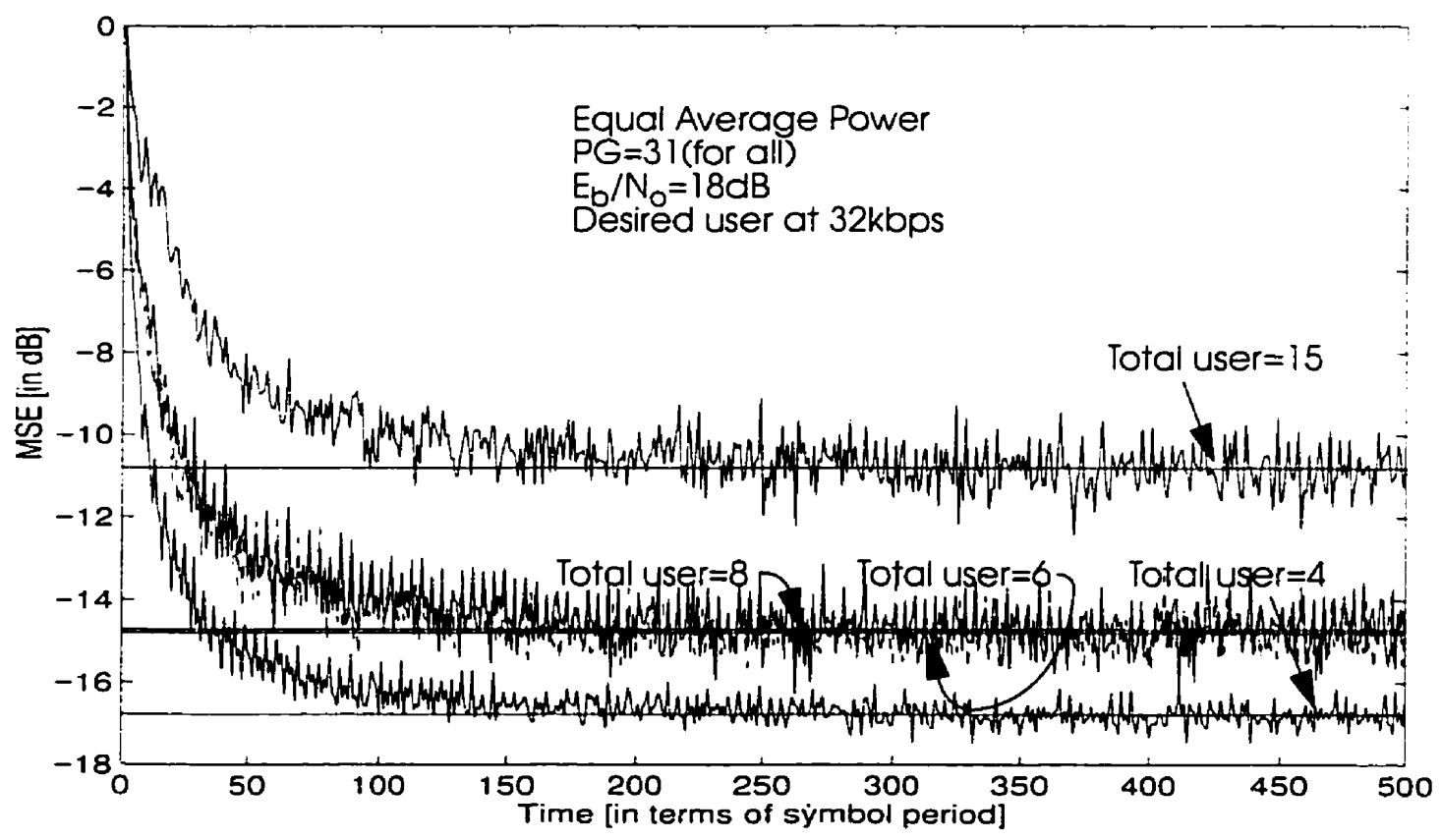

Figure 4.9: MSE Performance in scenario 1 
As described in section 3.3, at a given time the sum of the instantaneous power of three paths that consists channel may not be equal to one that is all paths may fade badly. This type of channel is termed as the "bad channel'. In simulation experiments. if BER is greater than $50 \%$ in any iteration, then that channel is considered as bad channel. Therefore, though the value of MSE is much better than the expected, but still the presence of bad channels may degrade the performance. This is seen by calculating the outage probability of the system. The outage probability due to MSE is defined as the ratio of number of channels for which the value of square error ' $s e$ ' is greater than the expected value of -6 $d B$ to the total number of channels. Figure 4.10 shows the probability of outage at $E_{H}$ $N_{0}=18 \mathrm{~dB}$ of the system for scenario 1 . In order to draw the outage plot the value of outage is determined every 10 th symbol after the training sequence. so the first symbol in the plot is the first symbol after the training sequence.

Outage plot in Figure 4.10 (according to the definition of outage probability). provides more confident estimate of outage probability compared to the case of smaller number of users where there are relatively few outage. As seen from the Figure 4.10. probability of outage is also very close for 6 and 8 users.

The probability of outage due to BER is shown in Figure 4.11. It is seen from figure that as the number of users increases the outage probability also increases which is expected. But 6 and 8 user case again performs close which confirms the finding of figures 4.8 through 4.10. To improve the estimate, simulation is performed for at least 150 and maximum 2000 individual channeis for each users. This means, for low $E_{b} / N_{0}$ and higher number of users the simulation is performed for 150 individual channels and for higher $E_{h} / N_{O}$ and less number of users the simulation is performed for 2000 channels. Due to this reason, in 


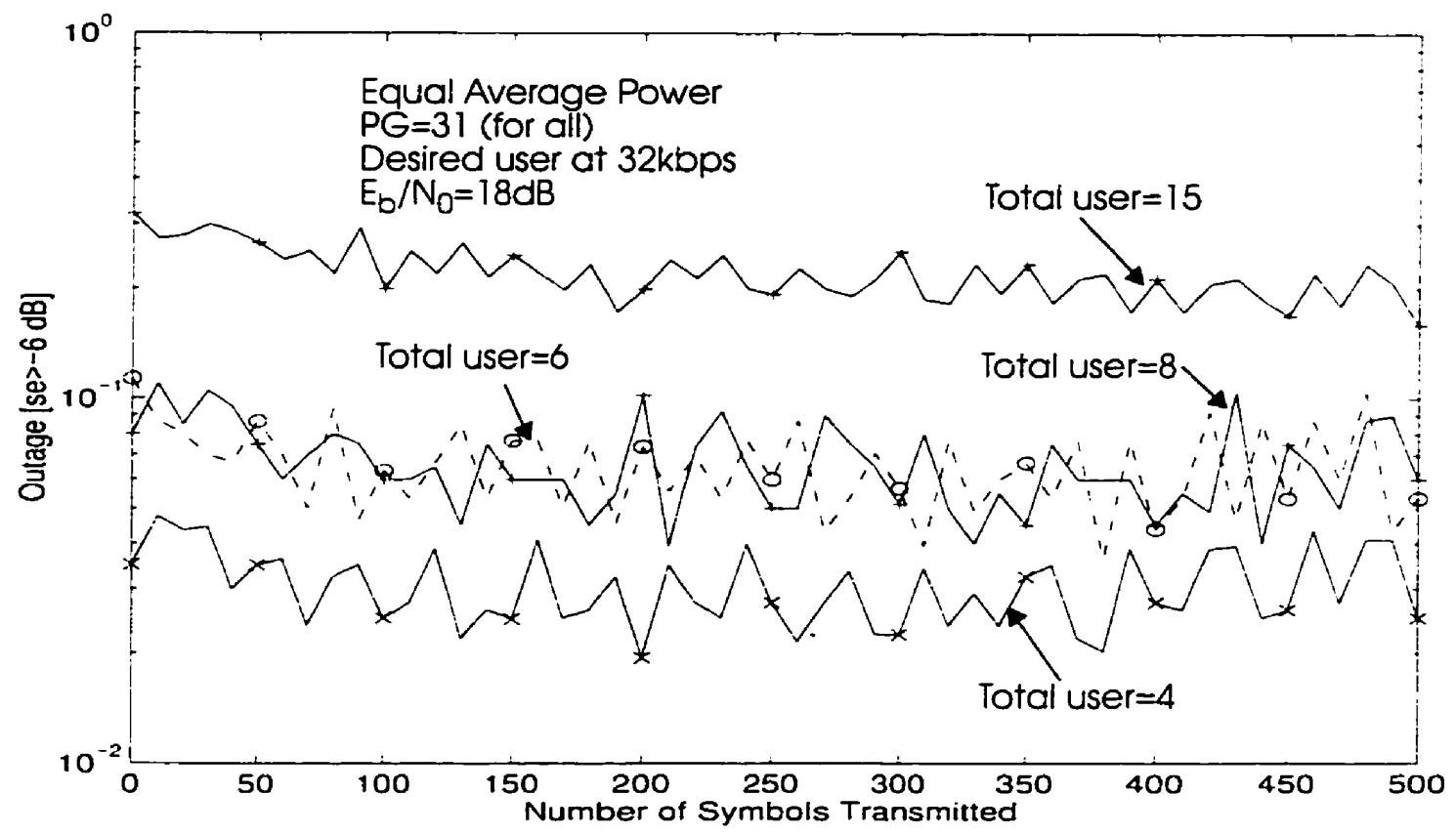

Figure 4.10: Probability of outage due to MSE in scenario 1

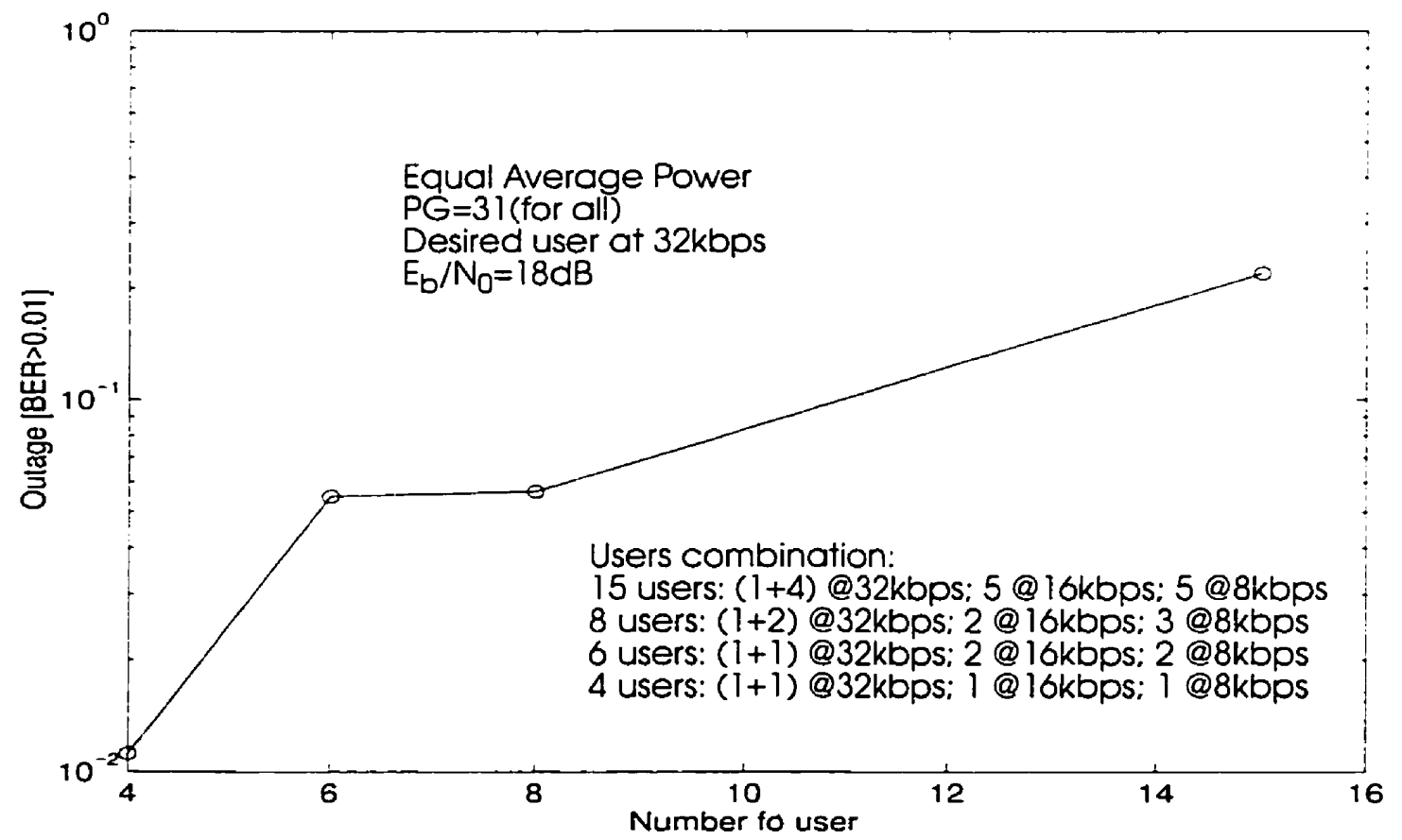

Figure 4.11: Probability of outage due to BER in scenario 1 
Figure 4.9, fluctuation in MSE for 15 user is higher than that of 4 users. This will be observed in rest of the MSE plots. MSE in each case is determined by taking average of last 250 symbols. This is true for the rest of MSE plots unless otherwise specified.

To observe the performance variation due to user combination in the presence of high interference, the system is simulated for 15 and 8 users only (scenario 2 ). The only difference between the scenario $I$ and 2 is the combination of interferers. BER and MSE performance for scenario 2 is shown in Figure 4.12 and 4.13. As seen from Figure 4.9 and 4.13. MSE performance between the scenarios are very close. which is also true in terms of outage probability due to MSE for the two scenarios as seen in Figure 4.15.

Applying equation (3.19) for 15 user case shows that performance for both scenarios should be almost same but scenario-1 slightly more immune to noise than scenario-2. But as seen from Figure 4.14, up to $16 d B$ scenario-2 is better than scenario-1. which may be dominant nature of noise than interference at low $E_{h} / N_{0}$. As $E_{h} / N_{O}$ increases. interference becomes the dominant factor and performance in scenario-2 began to degrade. Overall performance for 15 user case may also be considered as statistically similar. On the other hand. expected performance of scenario- 2 for 8 user case is noticeable over entire range of $E_{h} / N_{0}(6 d B$ to $30 d B)$. 


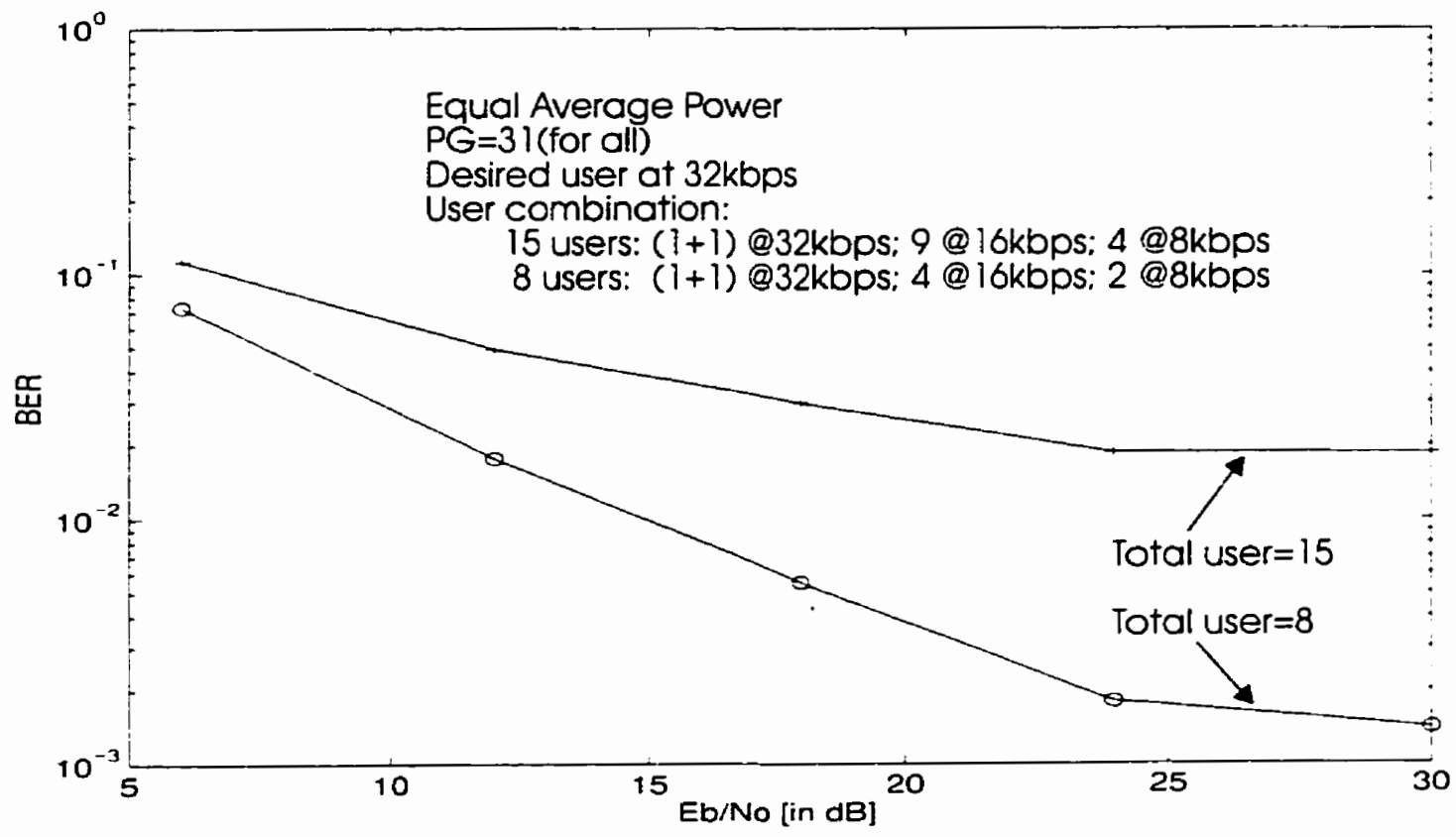

Figure 4.12: BER performance of scenario 2

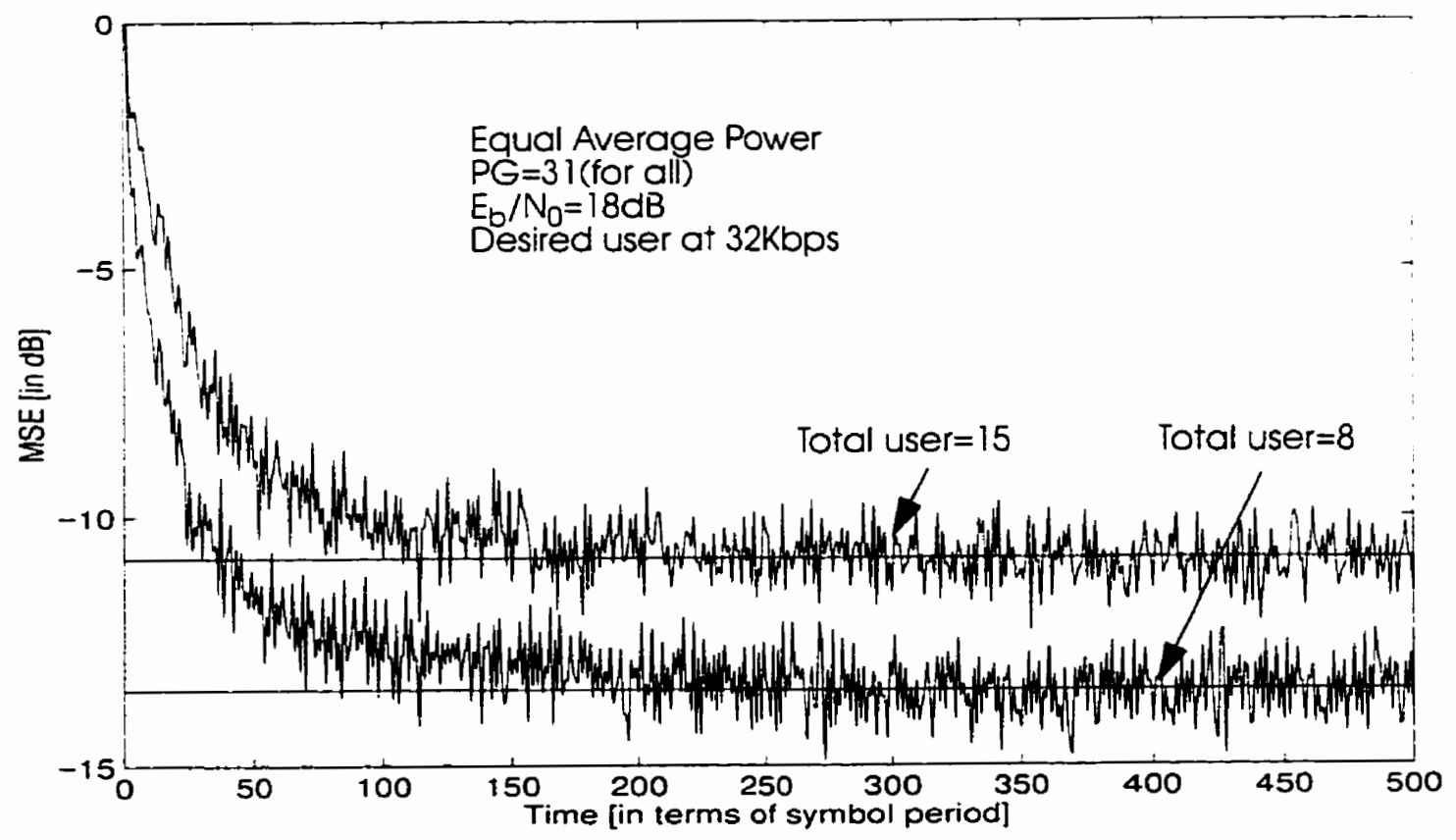

Figure 4.13: MSE performance of scenario 2 


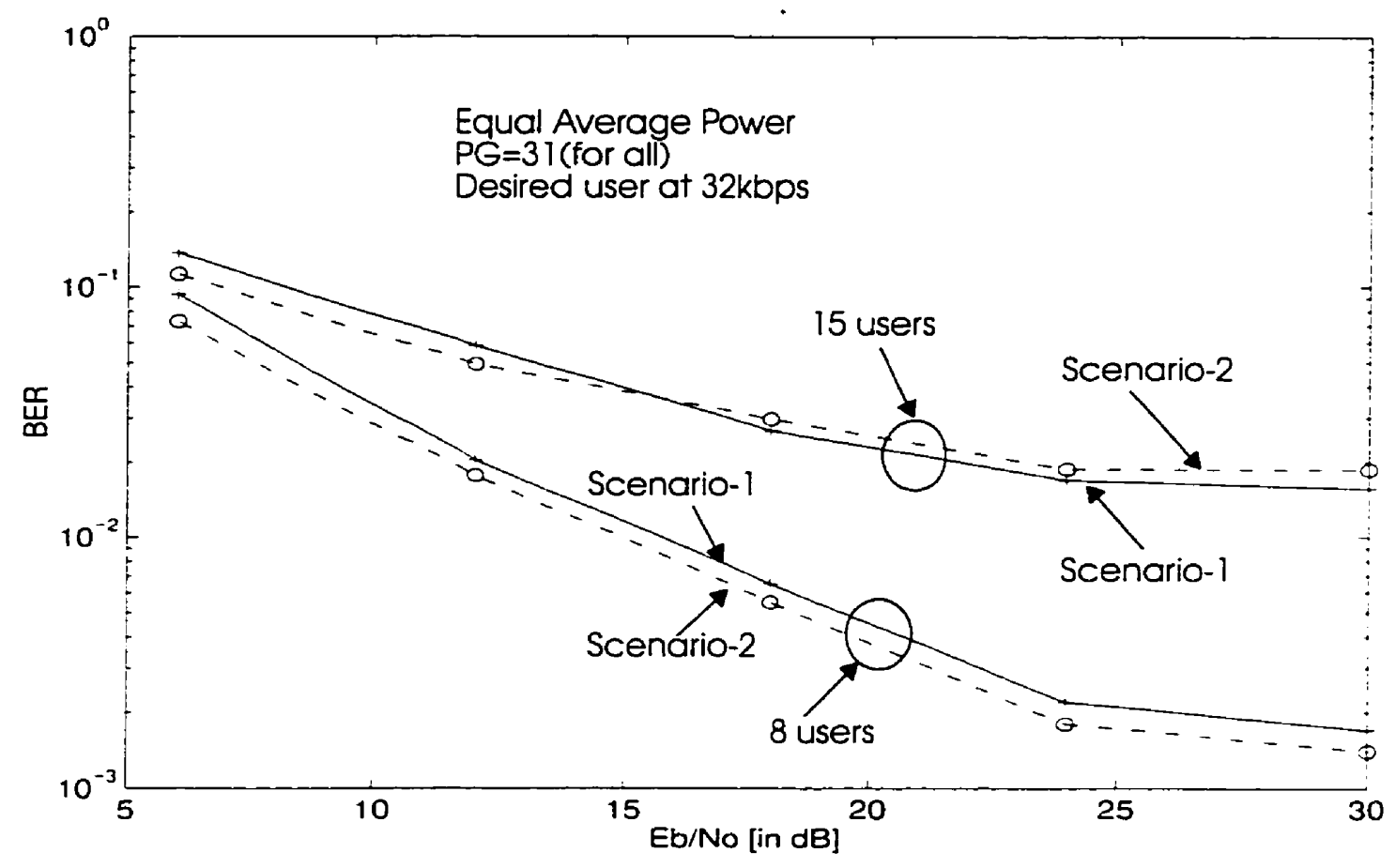

Figure 4.14: Comparison of scenario 1 and 2 in terms of BER

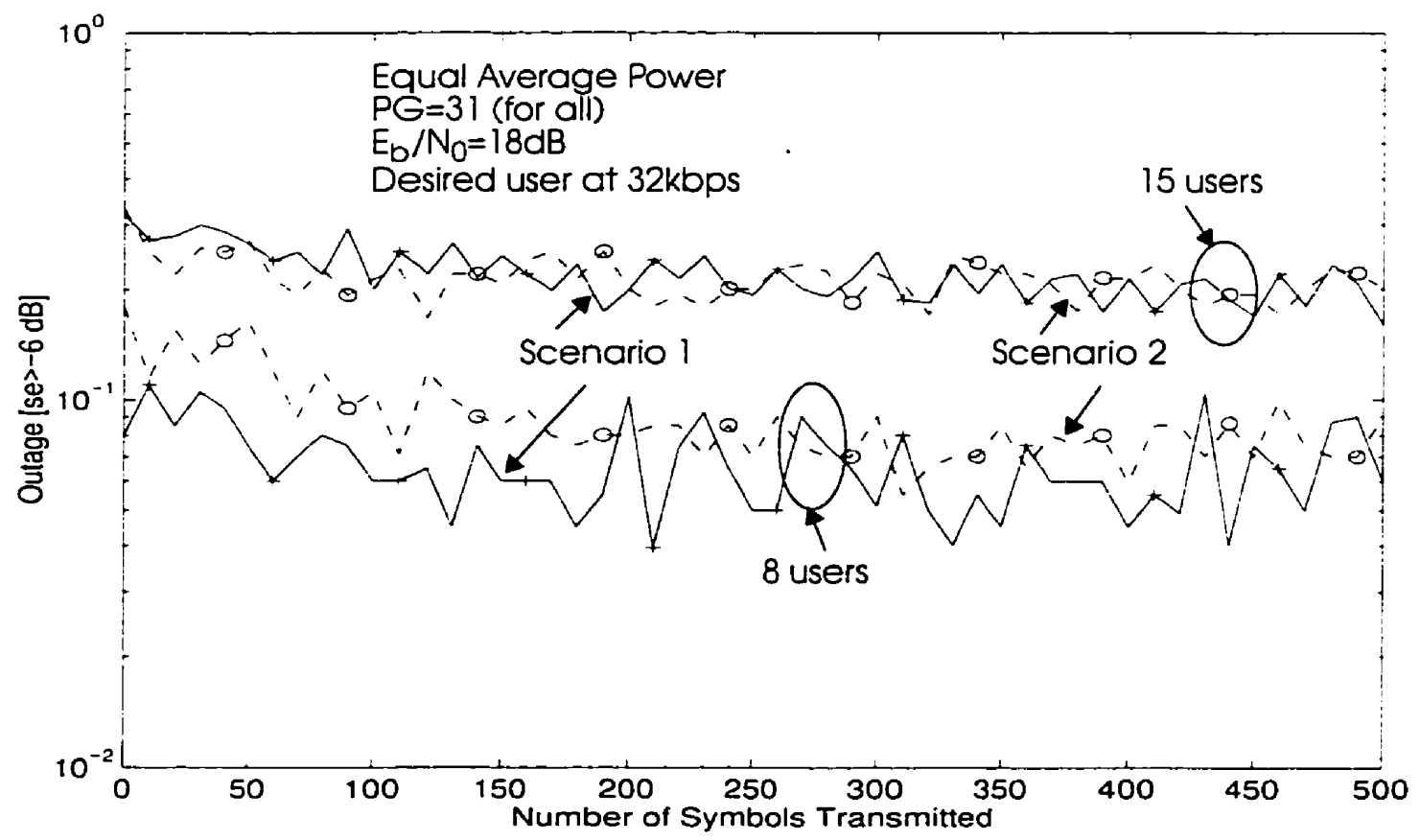

Figure 4.15: Comparison of scenario 1 and 2 in terms of MSE outage probability 


\subsubsection{Desired user at L-data rate}

This scenario presents the case in which the desired user is transmitting at $16 \mathrm{kbps}$ and interference is coming from a combination of users as shown in Table 3.1 (scenario 3 and 4). Simulation environment in this case is kept the same as that of scenario I and the plots are shown in Figure 4.16 through 4.23. The only difference between scenario $I$ and 3 is the desired user's signal, which is at L-data rate in this case.

As seen in Figure 4.16. BER performance improves as the number of users decreases. which is expected. However, up to $12 d B$, performance for 4.6 and 8 users are same. but at higher $E_{h} / N_{O}$ as interference is the dominant factor, performance at lesser number of users increases gradually. This performance improvement is also seen in terms of MSE as shown in Figure 4.17. The plots for probability of outage are shown in Figure 4.18 and 4.19 .

As the combination of interference is changed (scenario 4), there is very little change in performance is observed. This is shown in Figure 4.20 and 4.21. Though the outage probability due to MSE is same for scenario 3 and 4 (figure 4.23 ) but their BER performance is dependent on the combination of interference (figure 4.22) as expected.

According to equation (3.20), for 15 user case, insignificant better performance is expected for scenario-3, which is seen in Figure 4.22. But for 8 user case, scenario-4 is favorable for better performance. which is taking effect at high $E_{b} / N_{0}$ region where interference is the dominant factor. The poorer performance of scenario-4 at low $E_{h} / N_{O}$ seems like due to the dominant effect of noise rather than interference on the performance. 


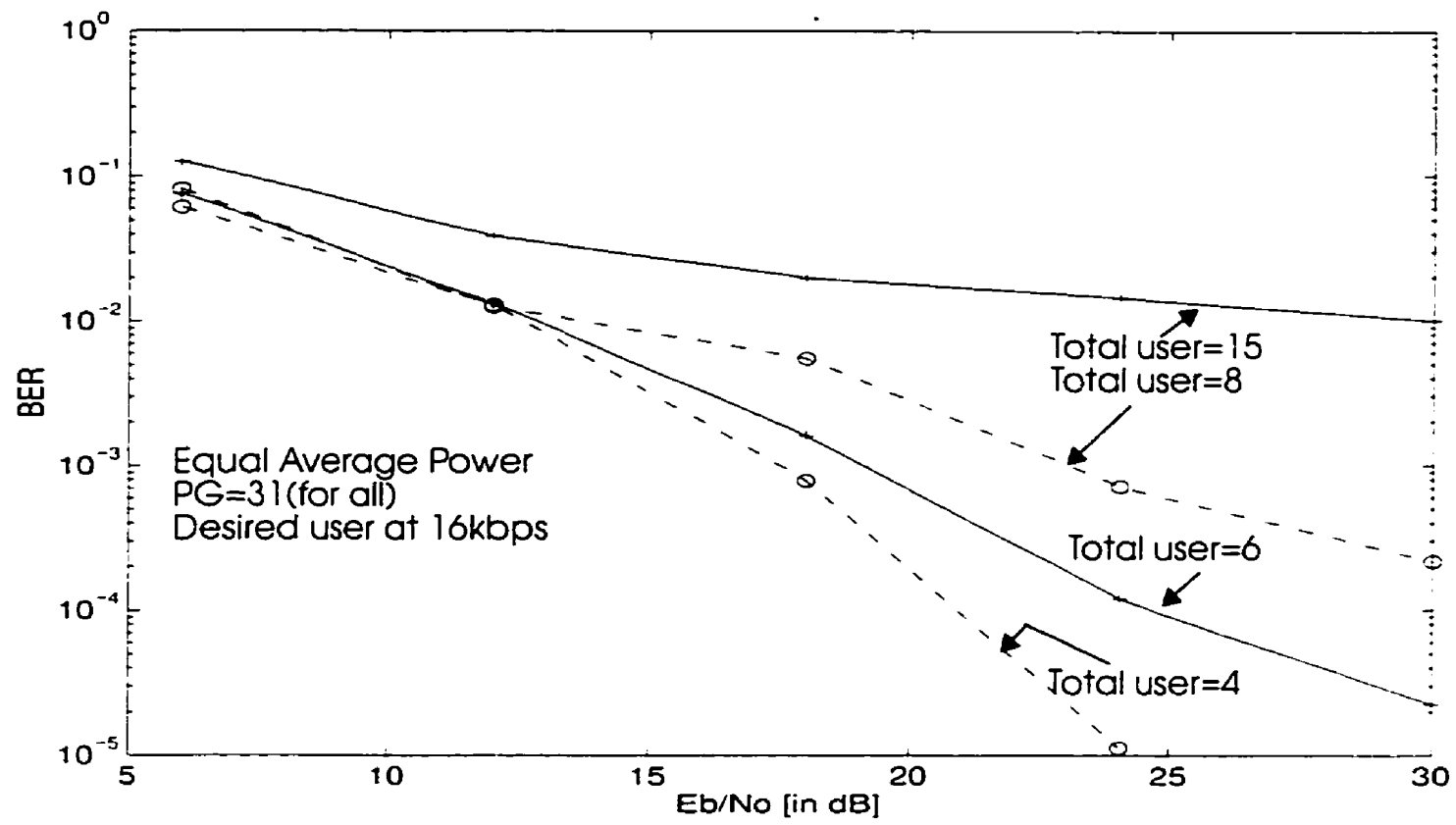

Figure 4.16: BER Performance in scenario 3

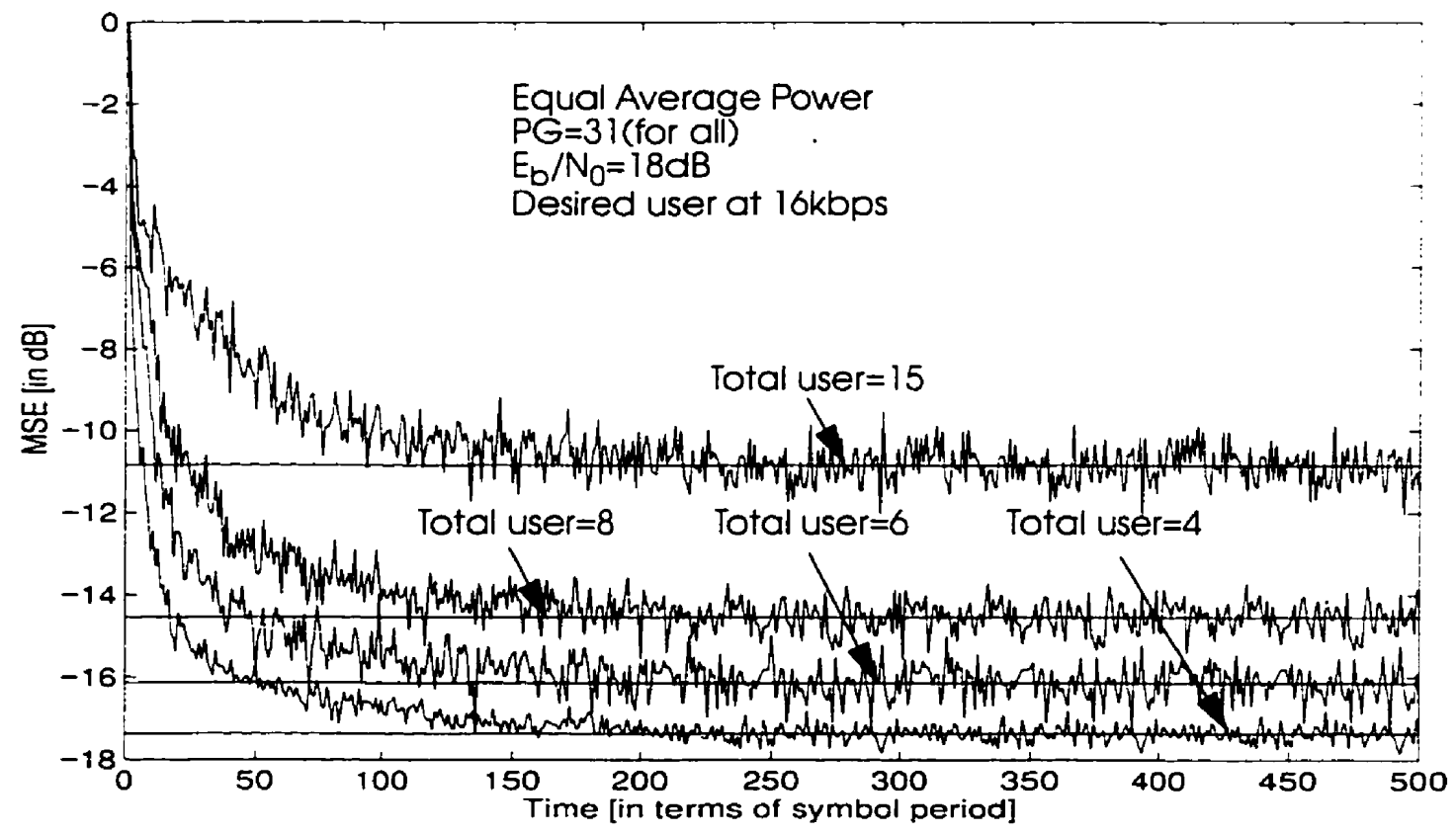

Figure 4.17: MSE performance in scenario 3 


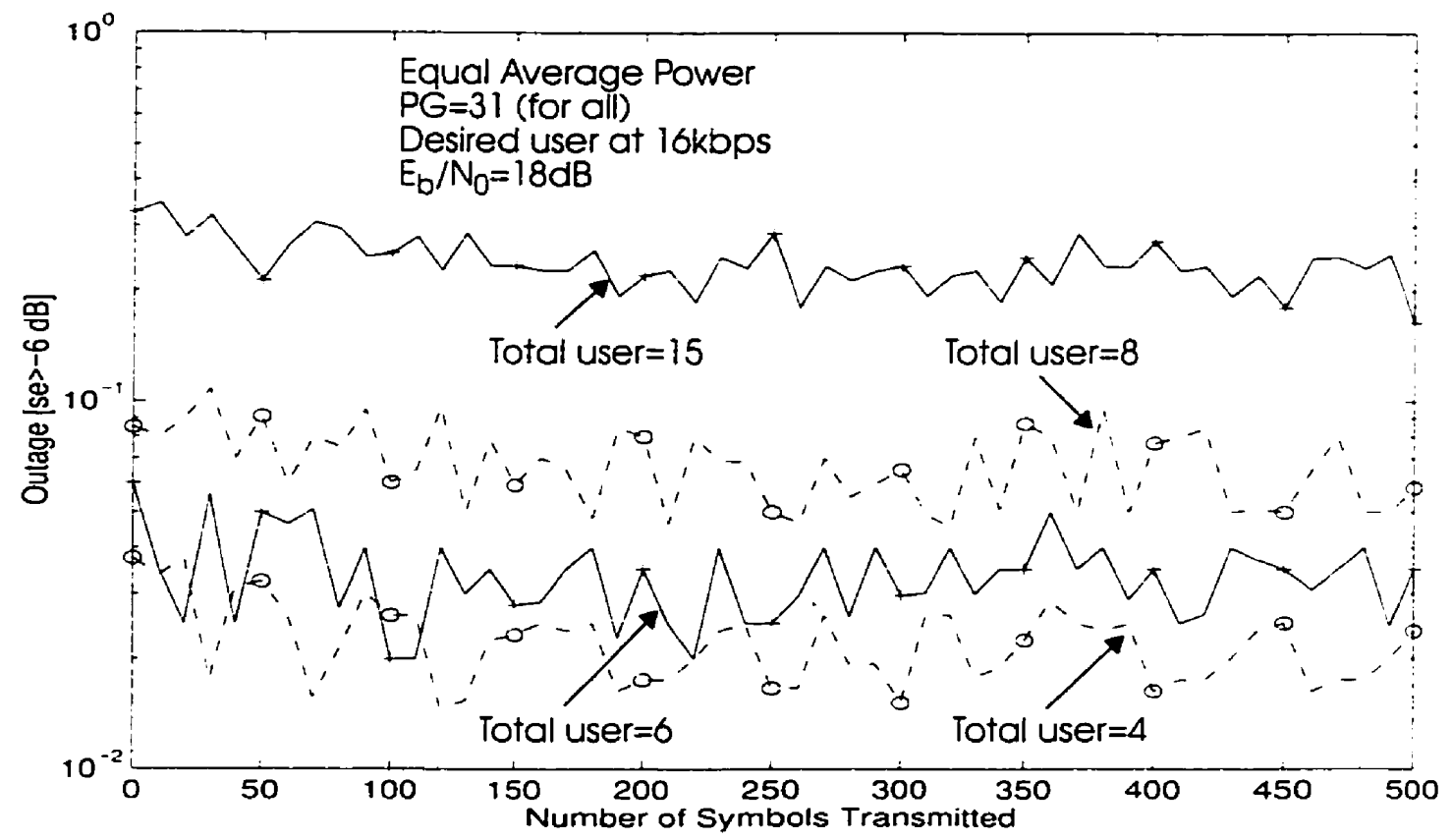

Figure 4.18: Probability of outage due to MSE in scenario 3

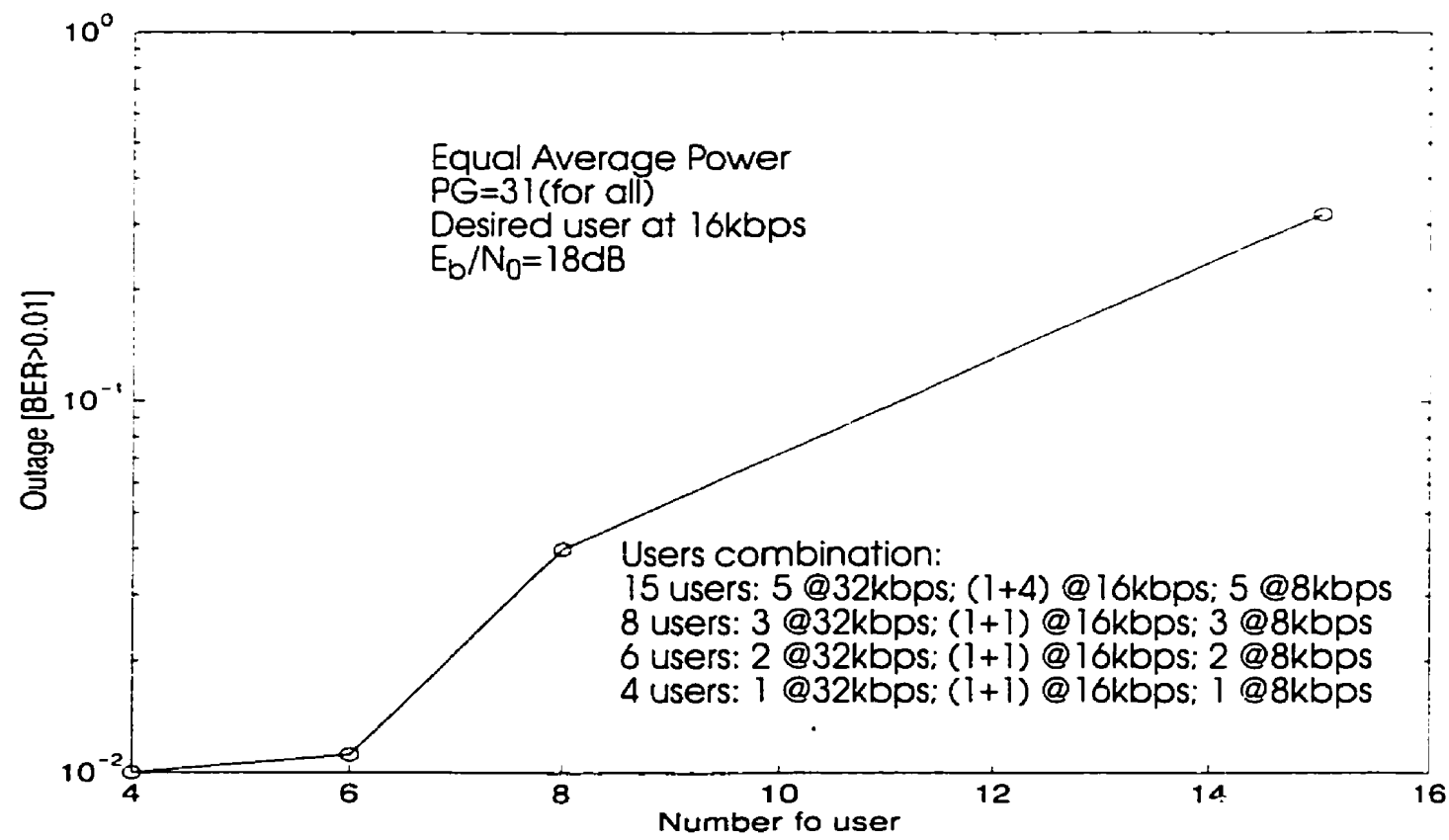

Figure 4.19: Probability of outage due to BER in scenario 3 


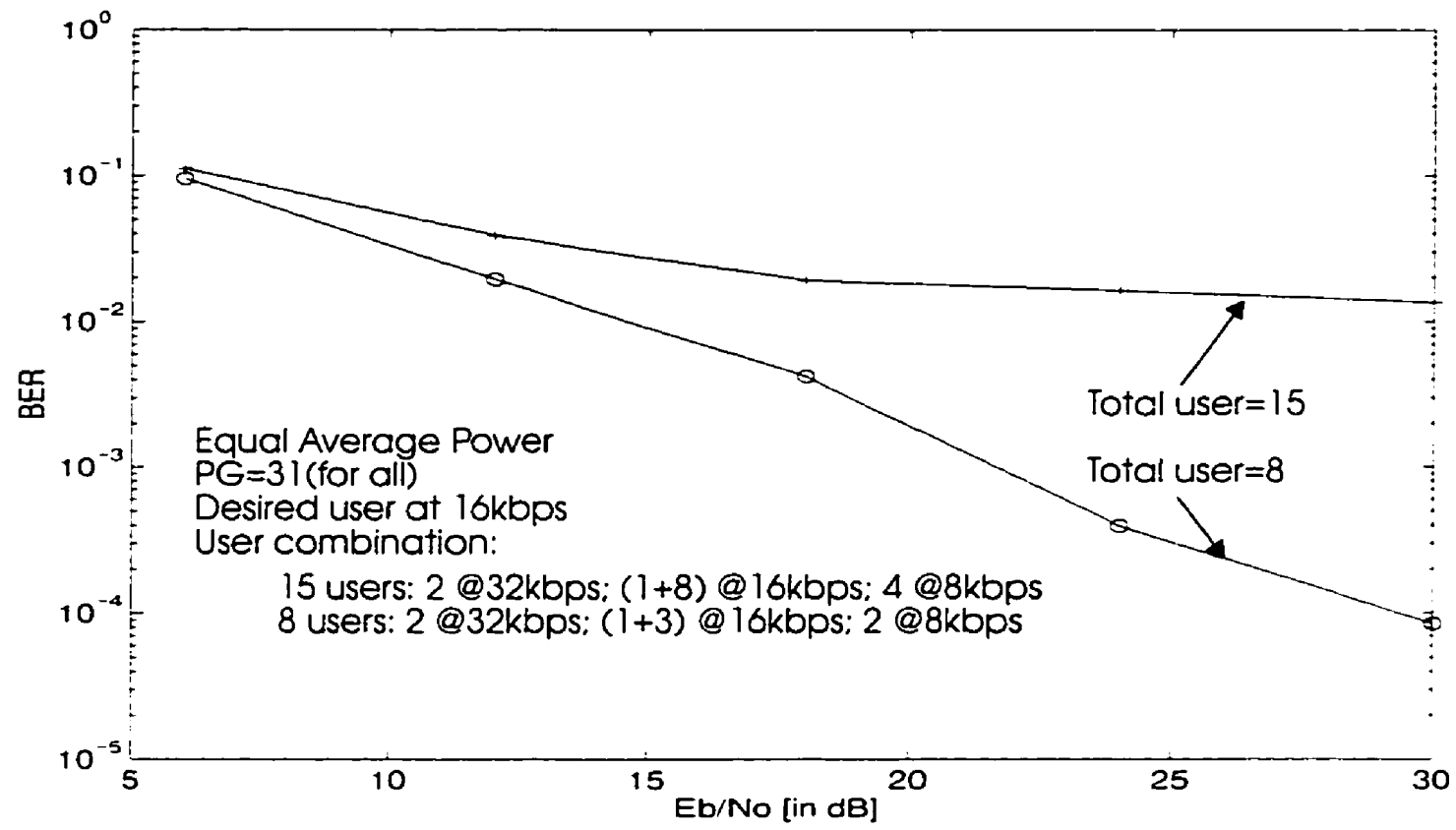

Figure 4.20: BER performance in scenario 4

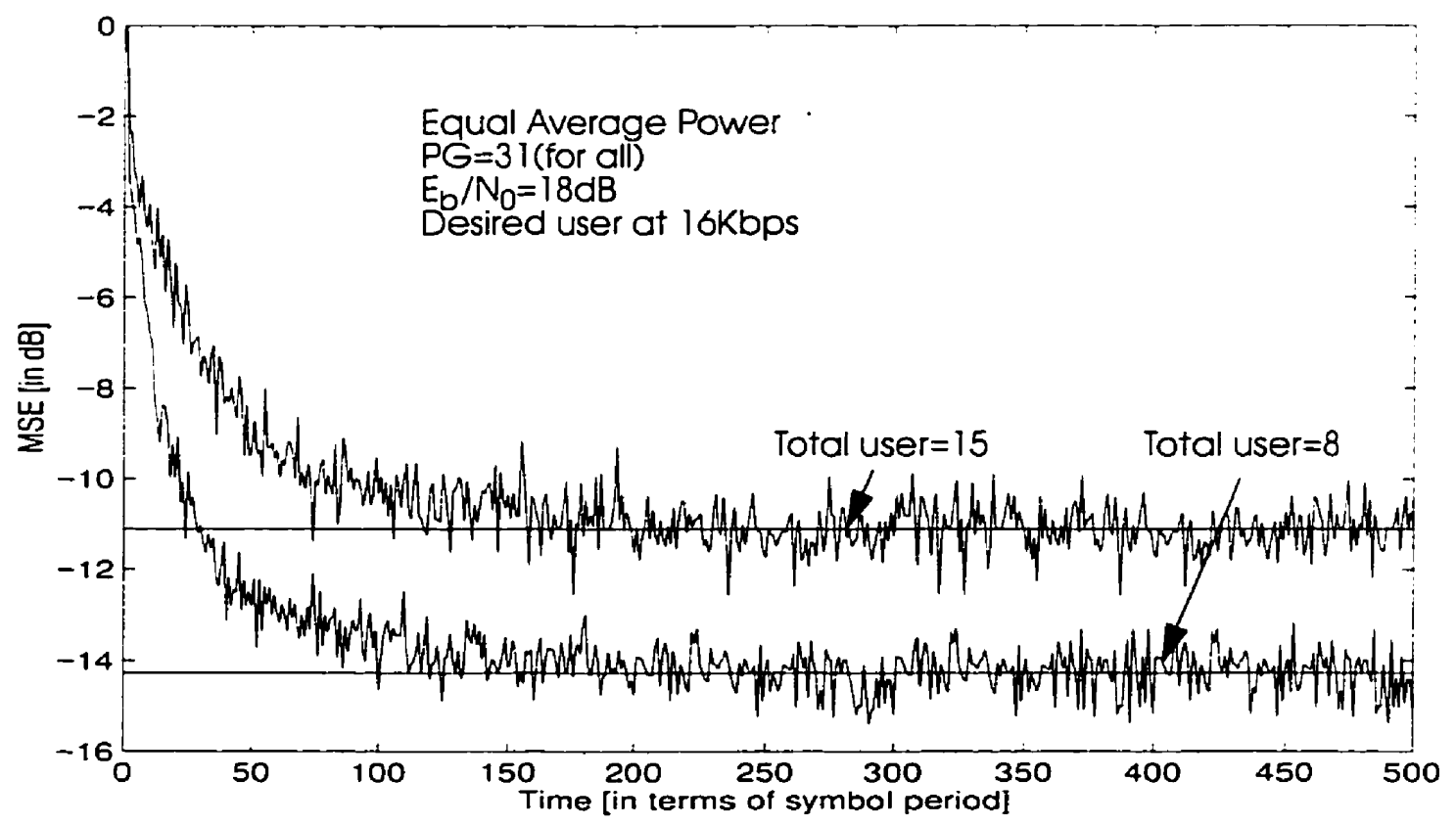

Figure 4.21: MSE performance in scenario 4 


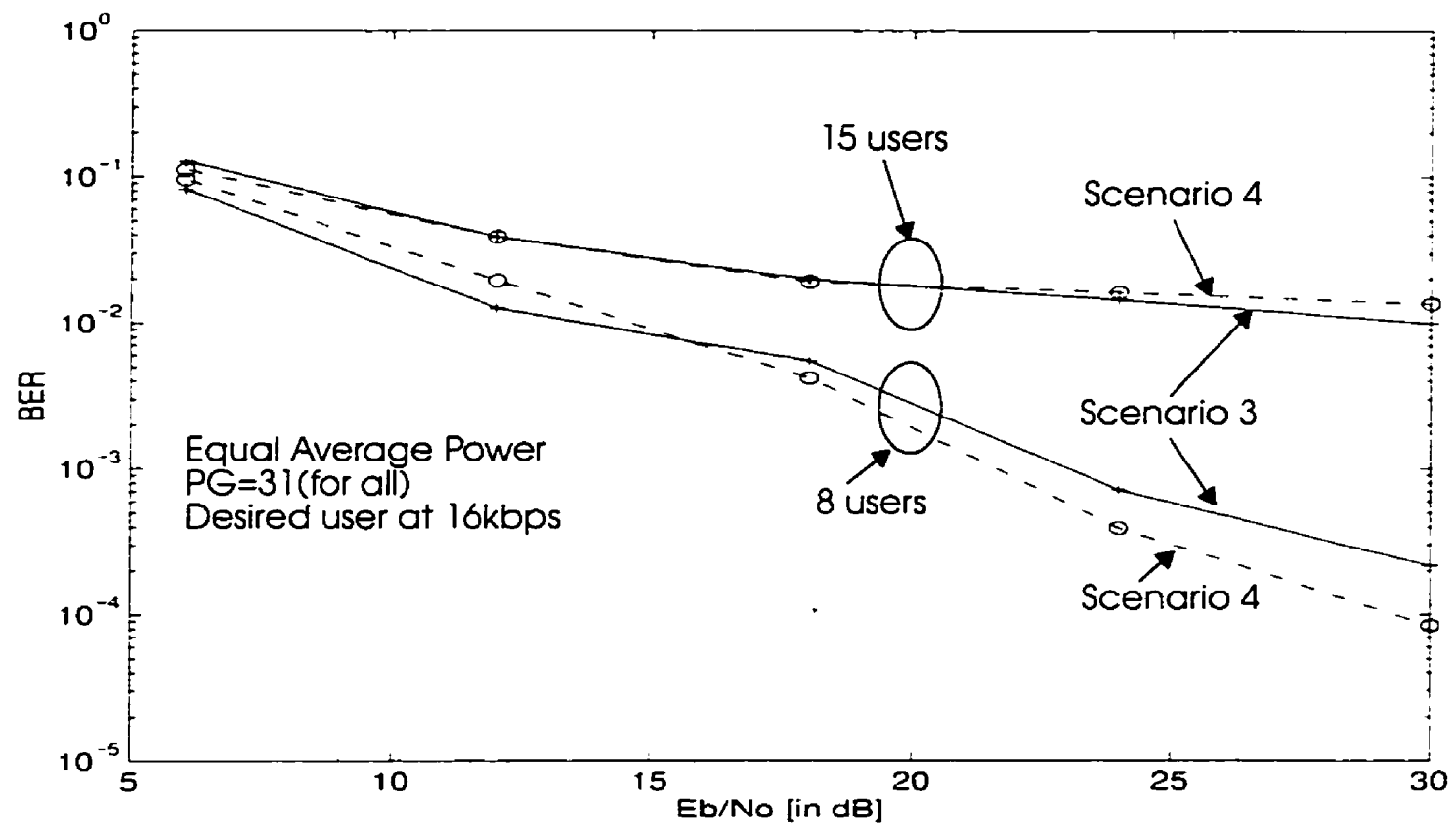

Figure 4.22: Comparison of scenario 3 and 4 in terms of BER

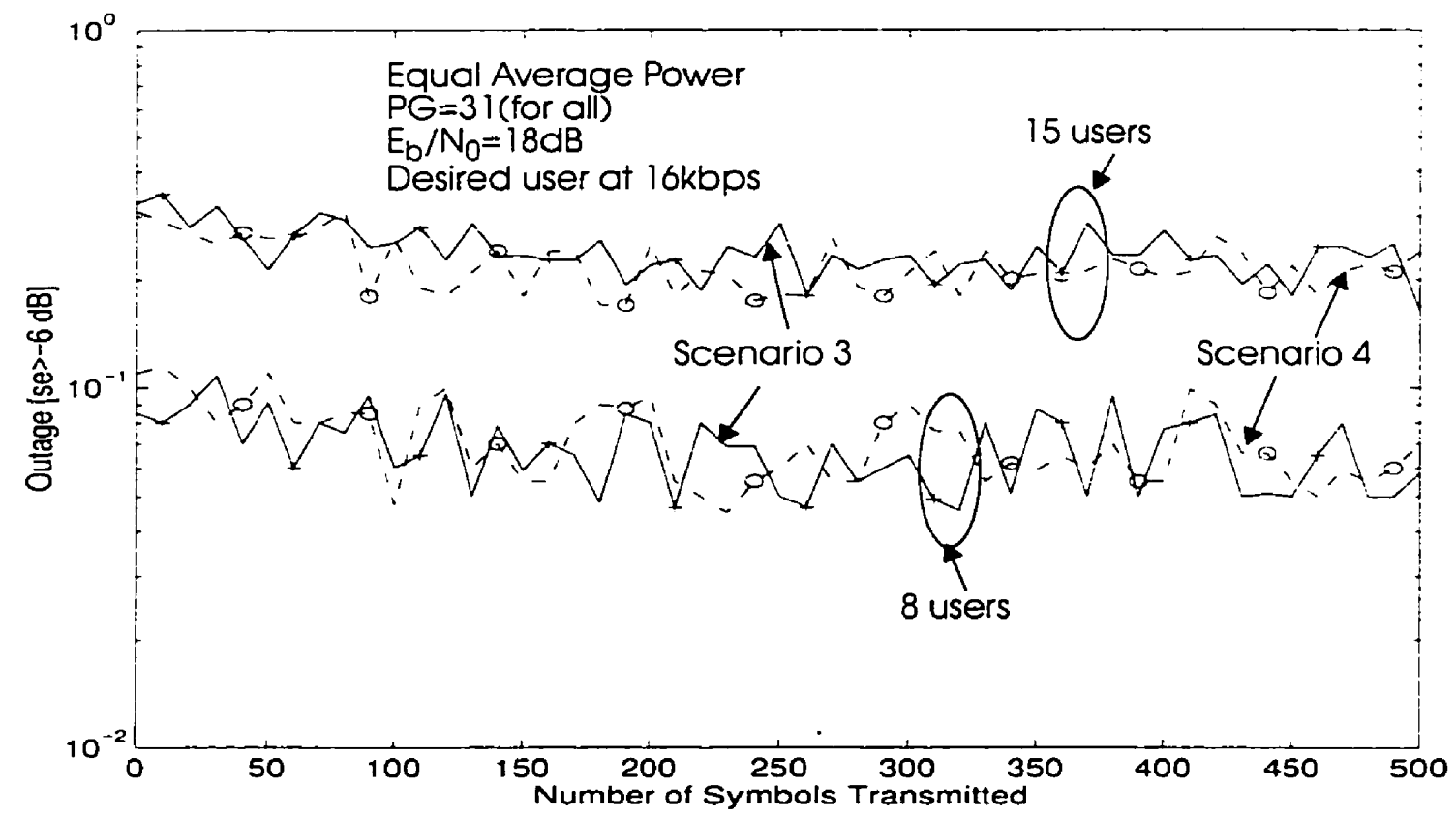

Figure 4.23: Comparison of scenario 3 and 4 in terms of MSE outage probability 


\subsubsection{Desired user at voice rate .}

This scenario presents the case in which the desired user is transmitting at $8 k b p s$ and interference is coming from a combination of users as shown in Table 3.1 (scenario 5 and 6). Simulation environment in this case is kept the same as that of scenario $l$ and the plots are shown in Figure 4.24 through 4.31.

As seen in Figure 4.24, up to $12 d B$, performance for 4.6 and 8 users are very close. because of noise, which is the limiting factor. But at higher $E_{b} / N_{\sigma}$ as interference due to undesired users power become the limiting factor, performance at lesser number of users increases significantly (referred to case-I, section 3.6). This performance improvement is also seen in terms of MSE as shown in Figure 4.25. The plots for probability of outage are shown in Figure 4.26 and 4.27. As the combination of interference is changed (scenario 6). there is very little change in performance is observed as expected. according to equation (3.2I). This is shown in Figure 4.30.

As explained in section 3.6 (case-I), for same combination of users. desired user at voice rate may perform better than that of desired user when selected from $\mathrm{H}$-data or L-data rate. The simulated result also agrees with this argument. This is shown in Figure 4.32 (for 15 user case) and 4.33 (for 8 user case) in scenario 1, 3 and 5. 


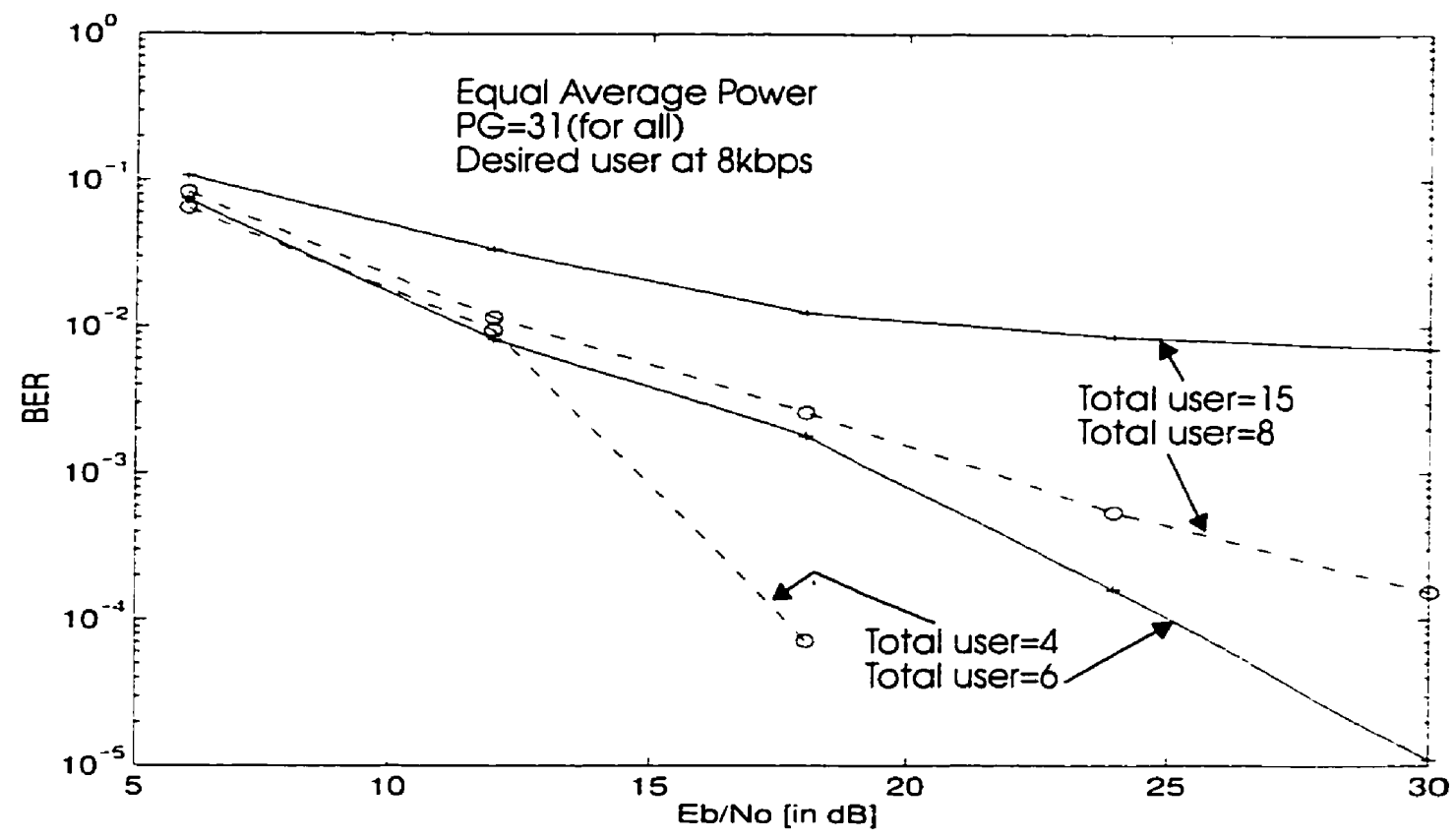

Figure 4.24: BER Performance in scenario 5

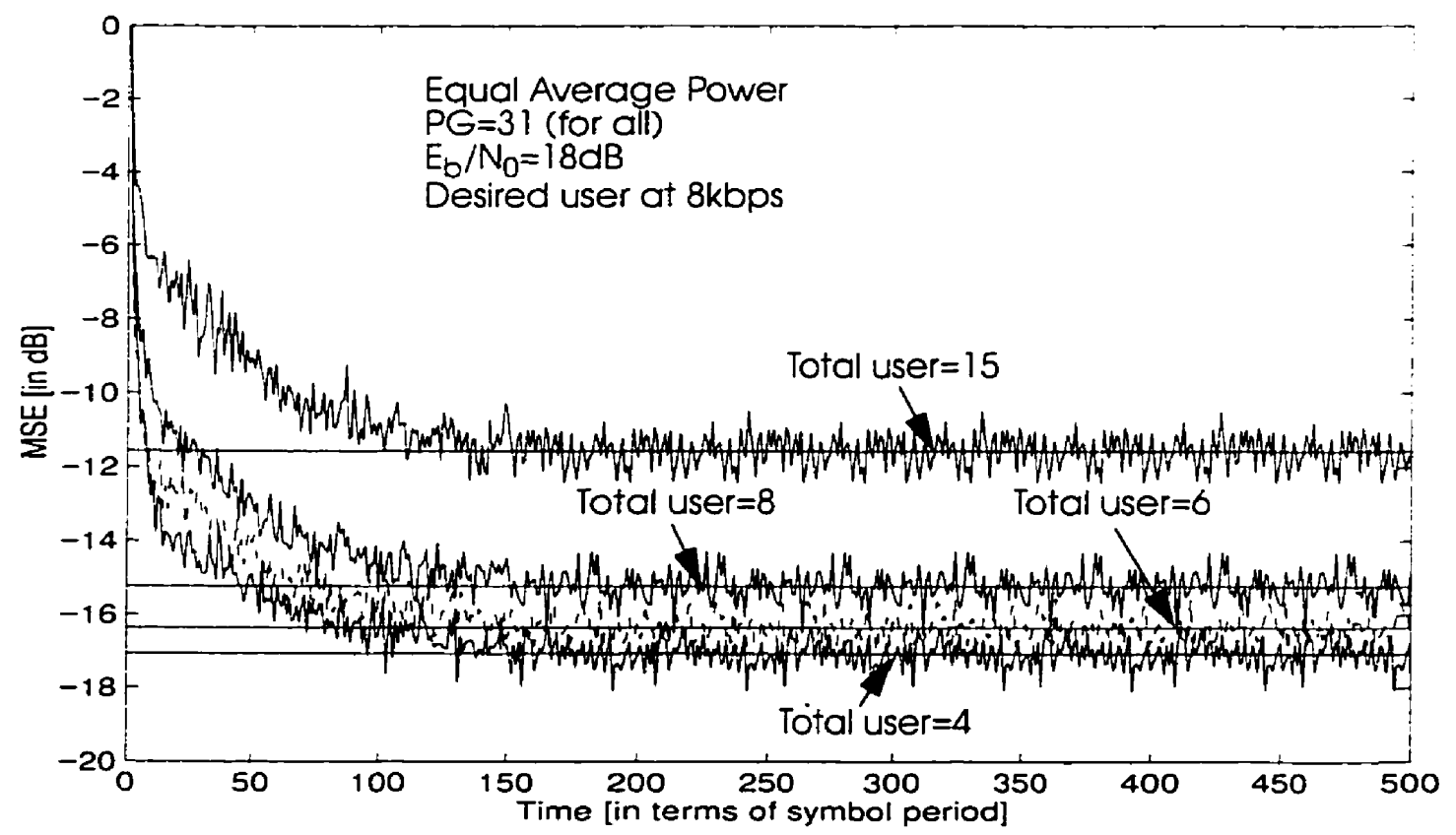

Figure 4.25: MSE performance in scenario 5 


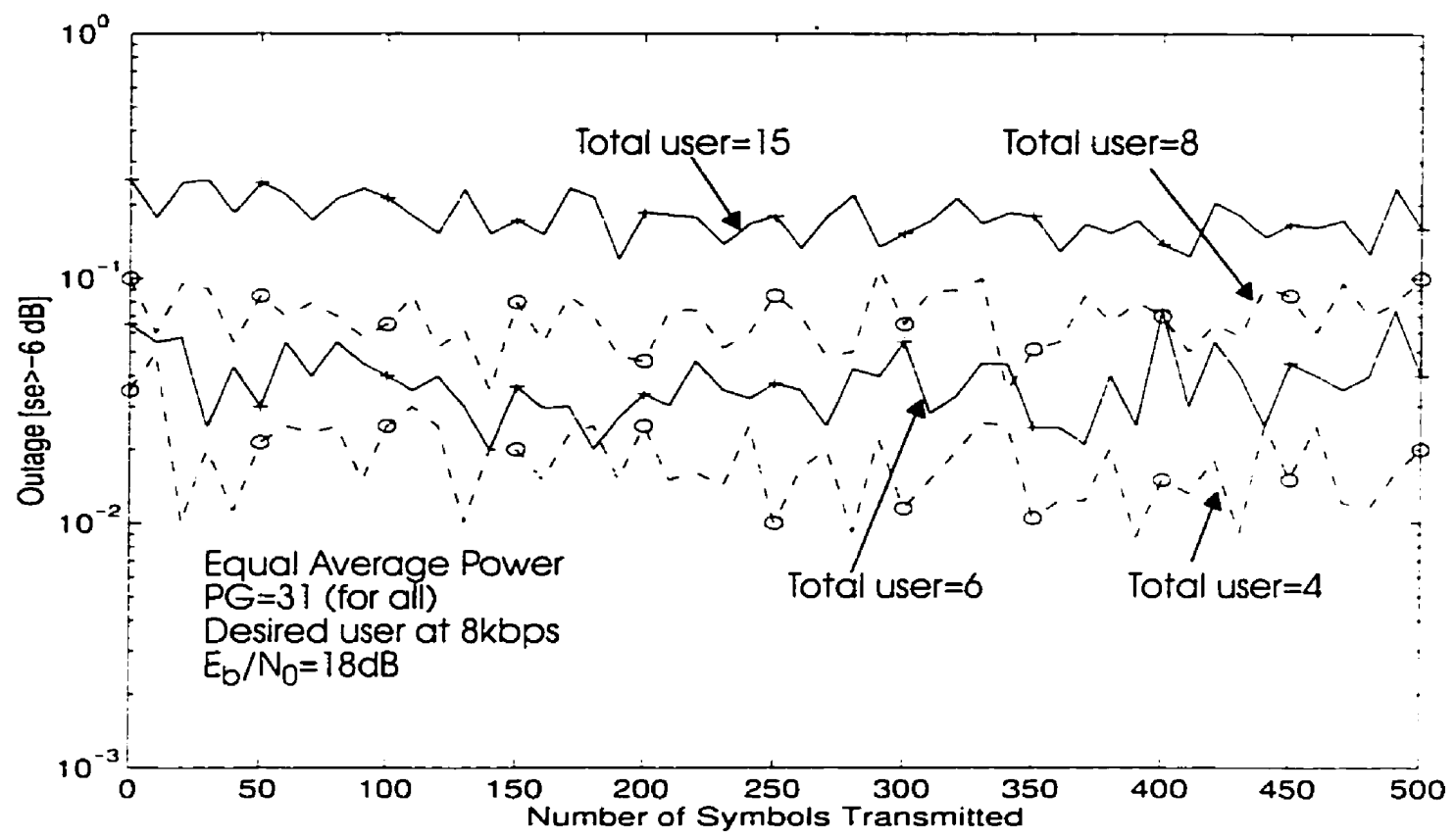

Figure 4.26: Probability of outage due to MSE in scenario 5

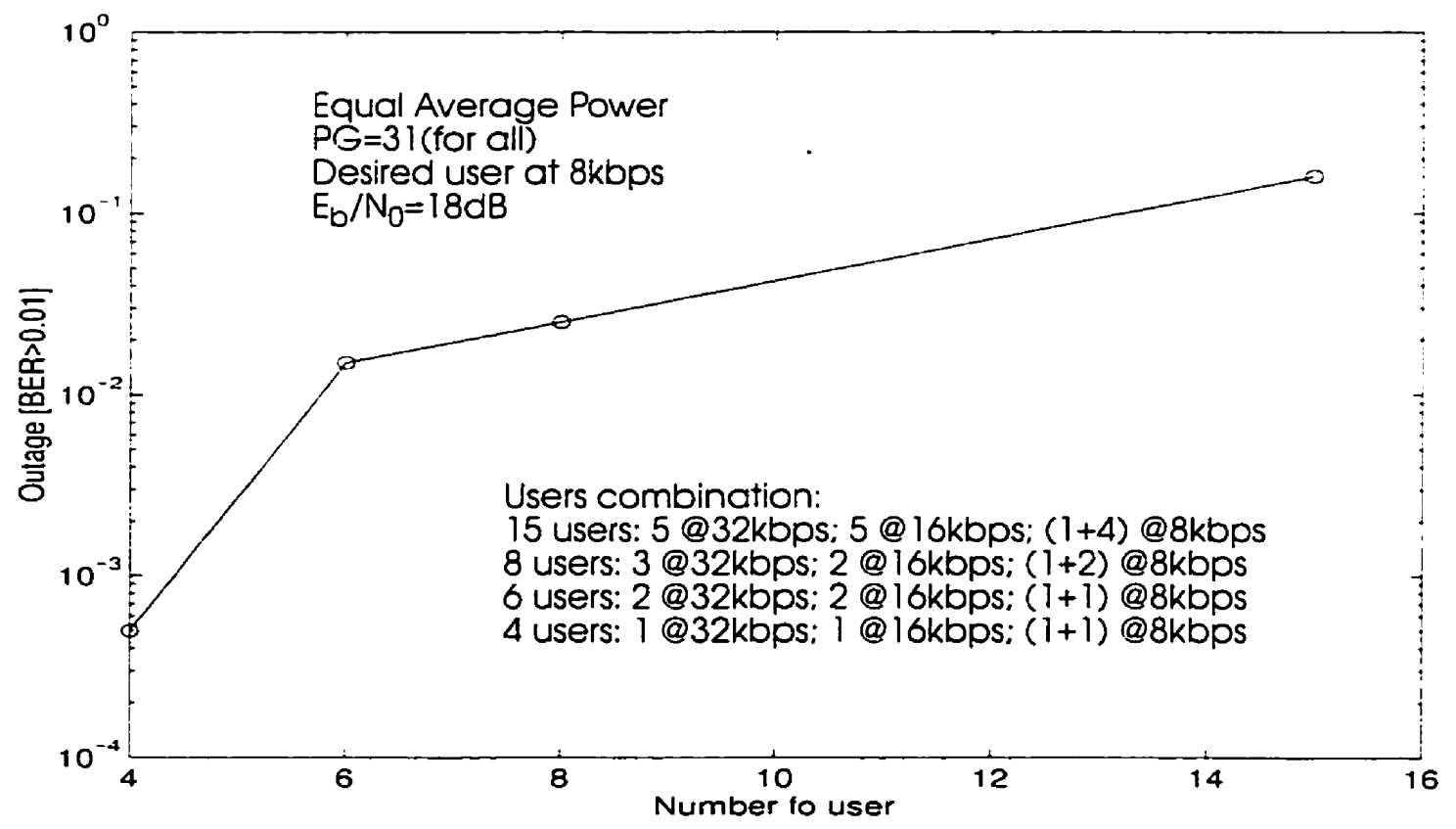

Figure 4.27: Probability of outage due to BER in scenario 5 


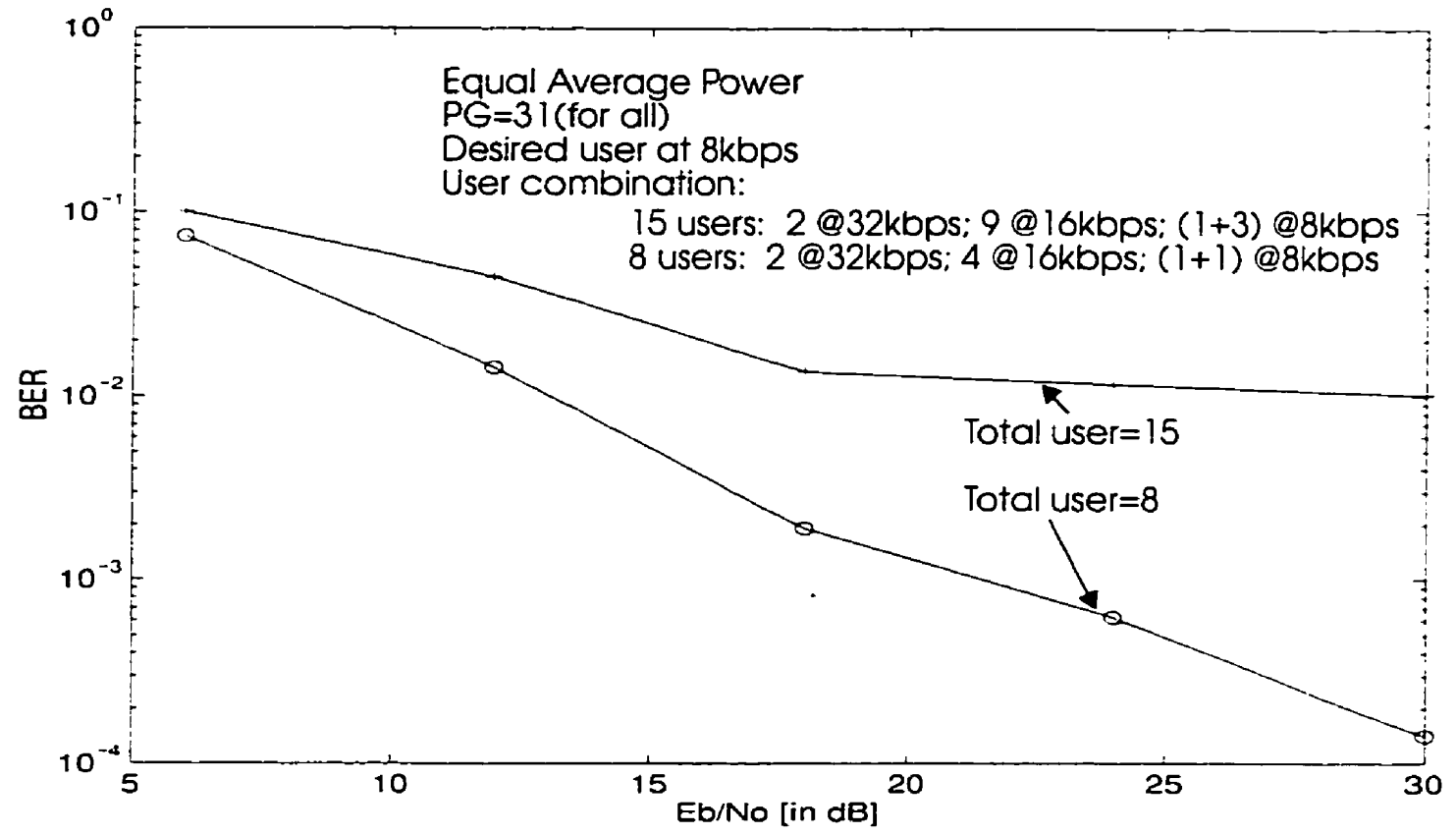

Figure 4.28: BER performance in scenario 6

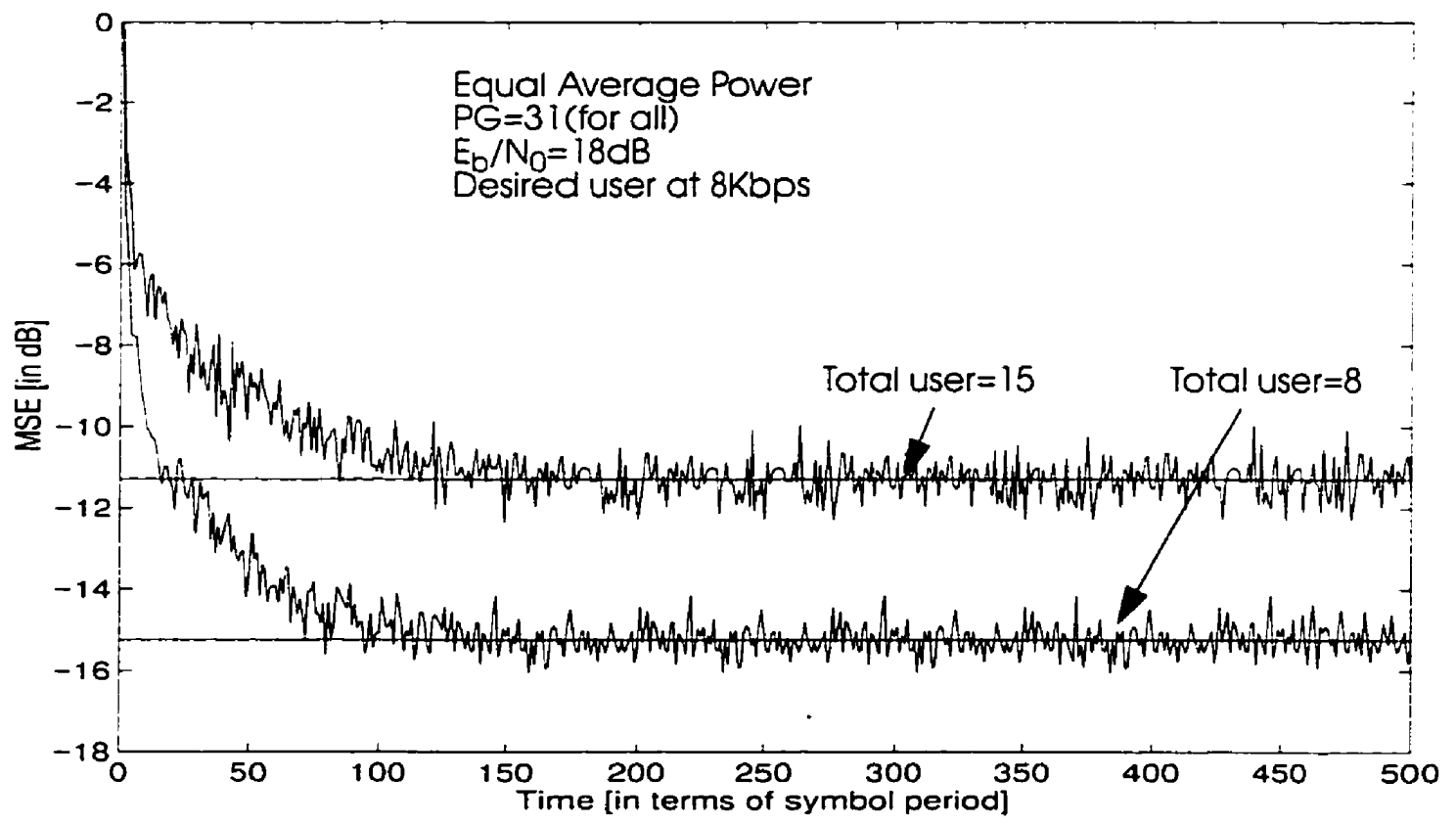

Figure 4.29: MSE performance in scenario 6 


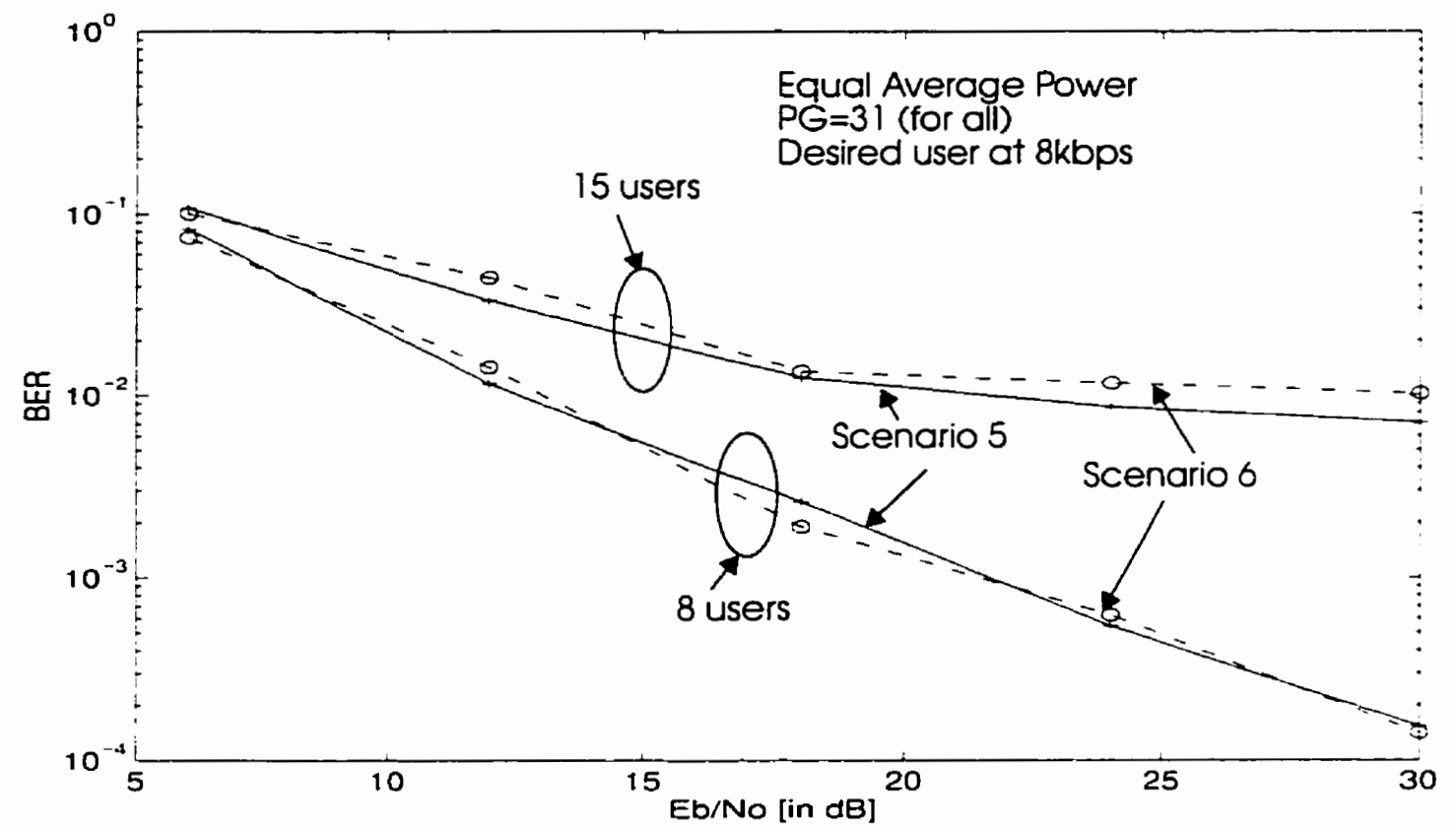

Figure 4.30: Comparison of scenario 5 and 6 in terms of BER

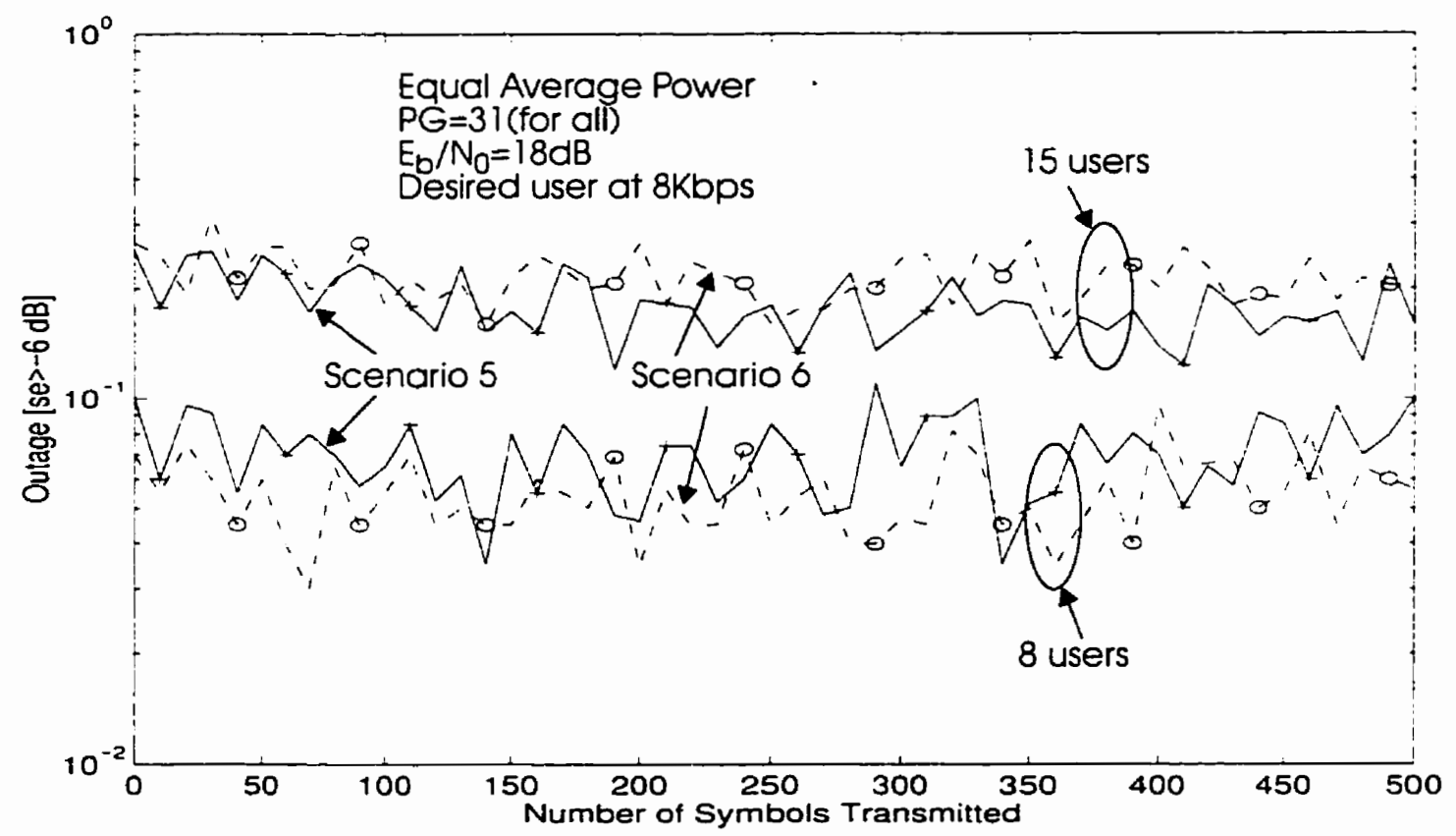

Figure 4.31: Comparison of scenario 5 and 6 in terms of MSE outage probability 


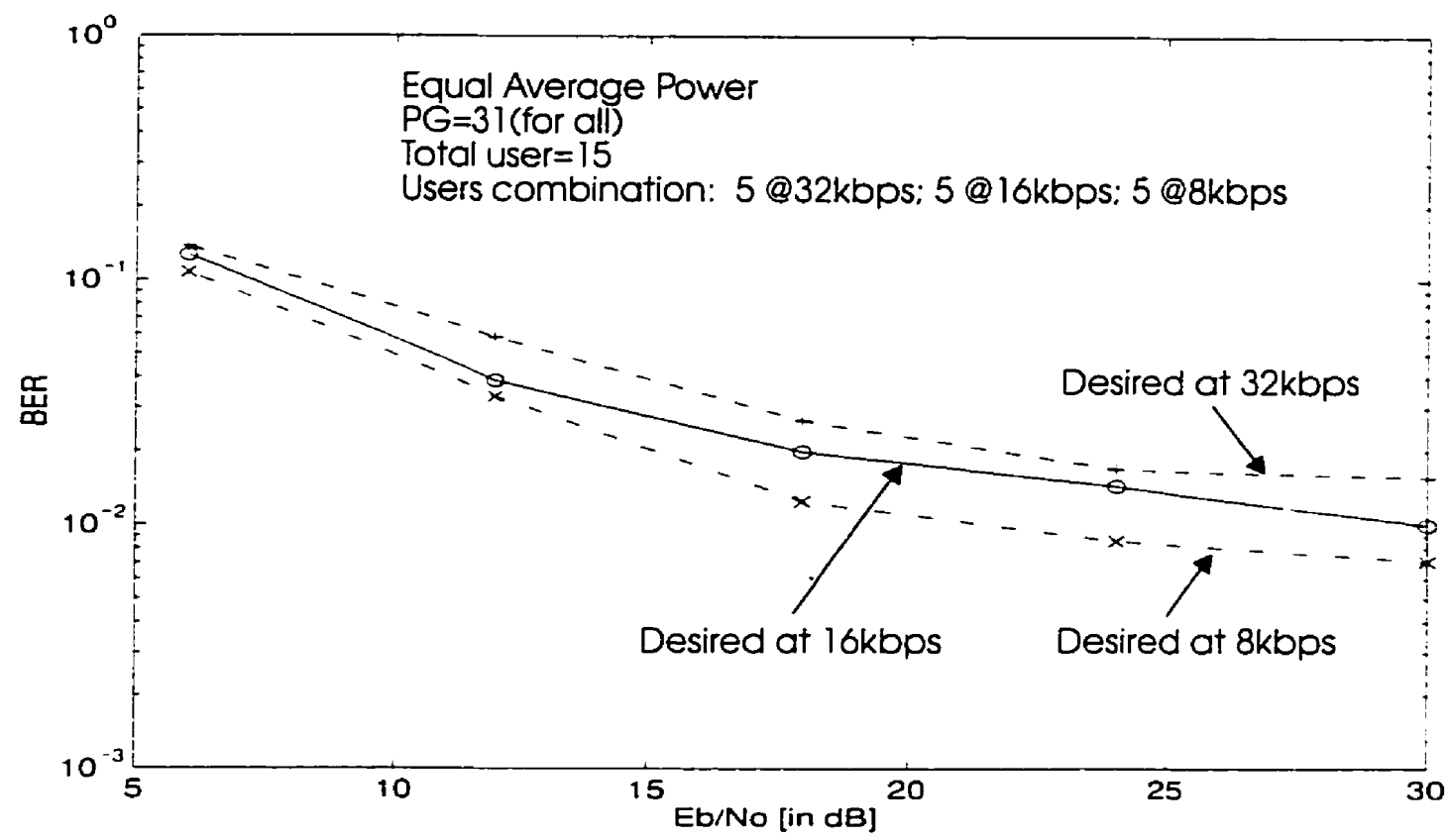

Figure 4.32: Comparison of 15 users case

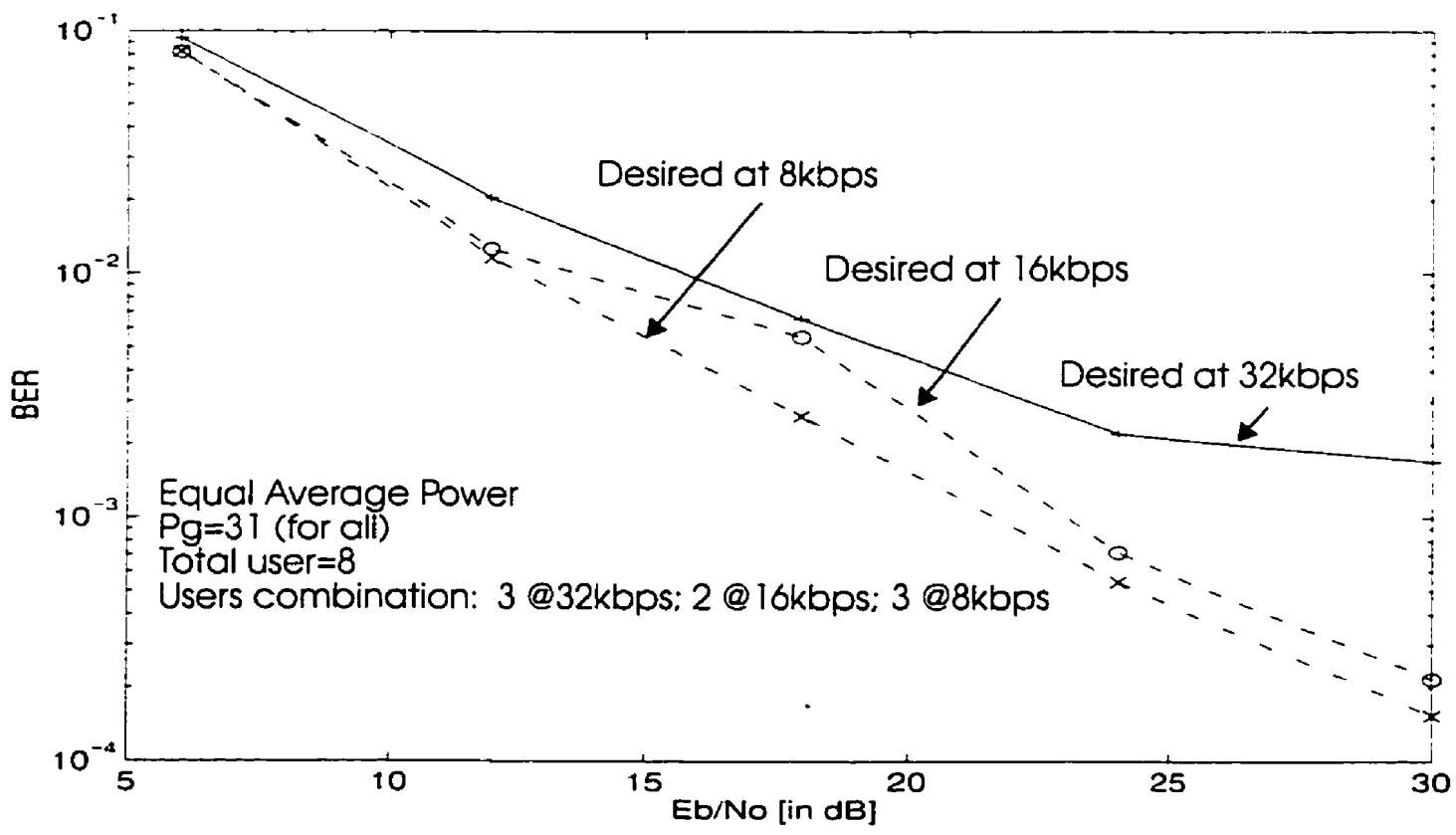

Figure 4.33: Comparison of 8 users case 


\subsubsection{Equal Bit Energy for each user}

This is the scenario where energy per bit for each user is kept equal regardless of their data rate. Other than this, the simulation environment is same as that of equal average power case. This situation is described in section 3.6. case-II. The simulation scenarios are the same as that of table 3.1. Following are the results when desired user is chosen from different data rate users.

\subsubsection{Desired user at $\mathrm{H}$-data rate}

As earlier, this scenario presents the case in which the desired user is transmitting at $32 k b p s$ and interference is coming from a combination of users as shown in scenario-I and 2 of Table 3.1. The plots for this case are shown in Figure 4.34 to 4.39 . From the figures the expected performance degradation is observed as the number of user increases. According to expression (3.23). expected improved performance is also observed for scenario-2 from Figure 4.38 .

Comparing Figure 4.8 and 4.34 shows that when desired user is selected from $\mathrm{H}$-data rate users, overall performance for equal bit energy case is much better than that of equal average power case. The reason is, since $E_{b}$ is same for all users, therefore. $\mathrm{H}$-data users has the advantage over voice rate users in terms of signal power. On the other hand. when signal power is same. $\mathrm{H}$-data users has the disadvantage in terms of $E_{h}$ (referred to section 3.6). This improved performance is also observed in terms of MSE. Outage probability due to MSE and BER is also better in the latter case which is shown comparing Figure 4.10, 4.11 and Figure 4.36, 4.37 respectively. In consistence. scenario-2 also performs better which is shown in Figure 4.38 and 4.39 in terms of BER and MSE respectively. 


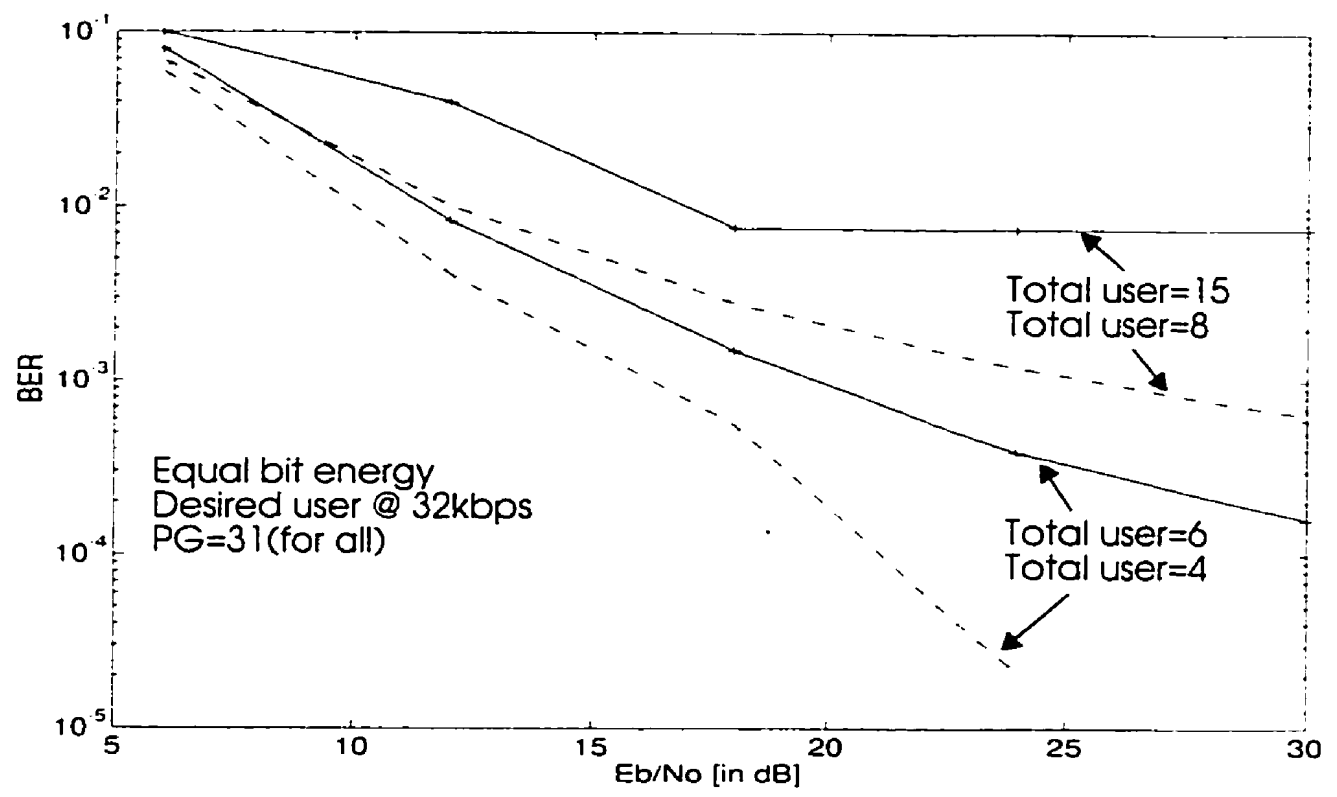

Figure 4.34: BER performance in scenario 1

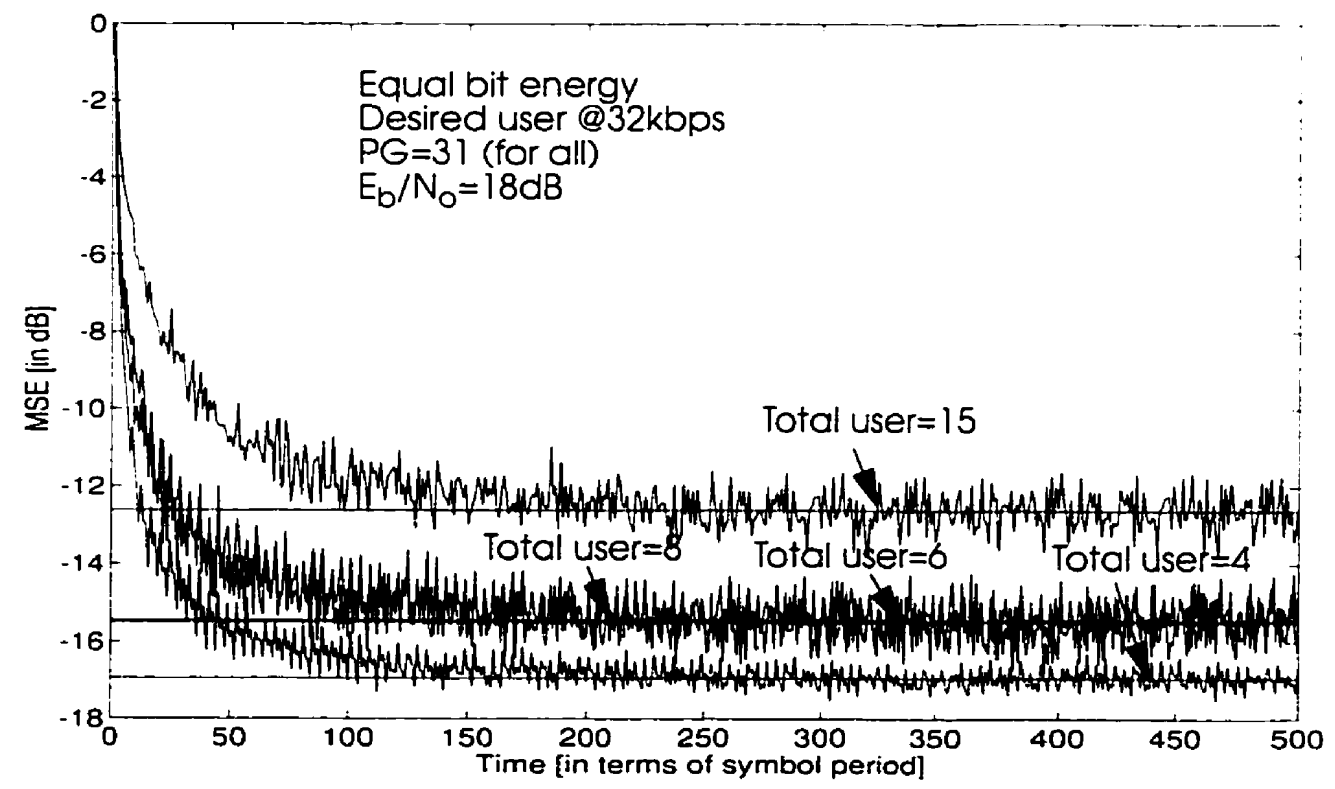

Figure 4.35: MSE performance in scenario 1 


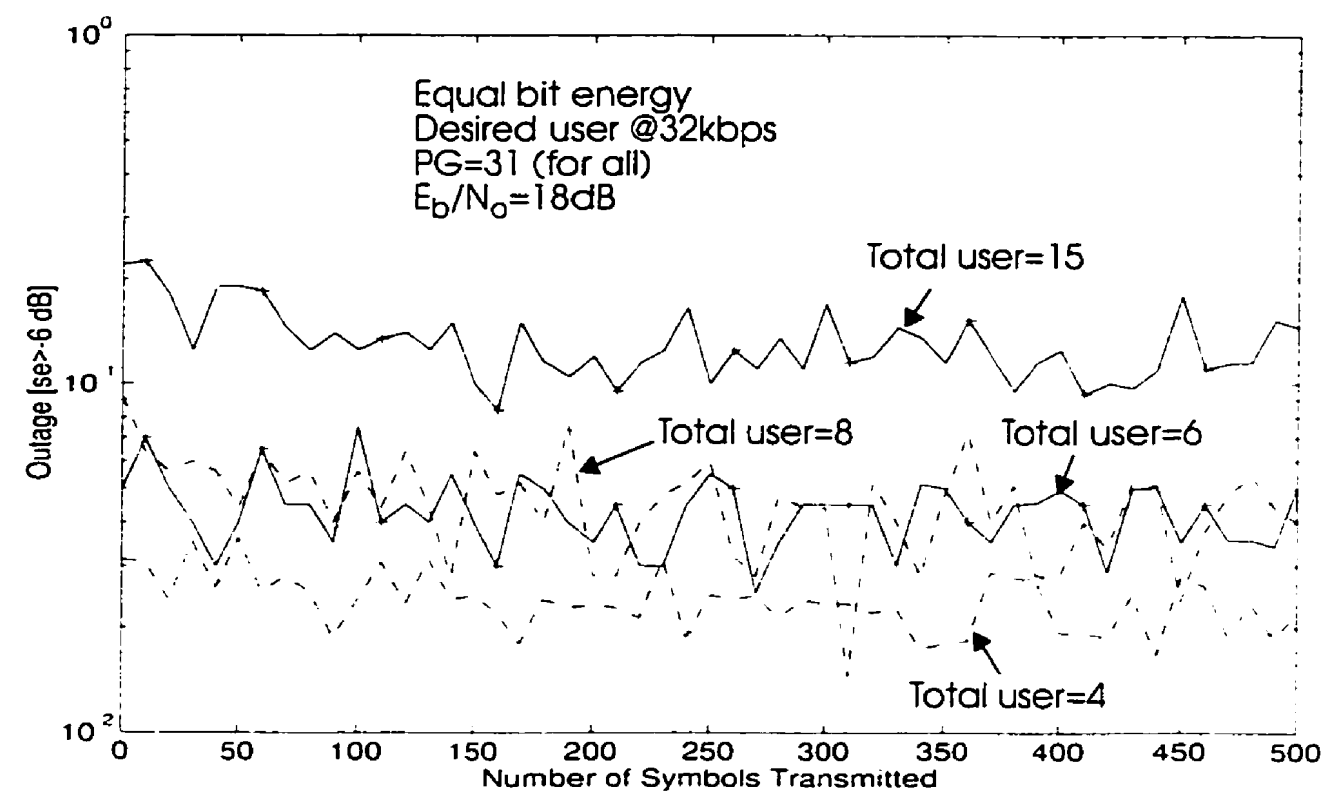

Figure 4.36: Probability of outage due to MSE in scenario 1

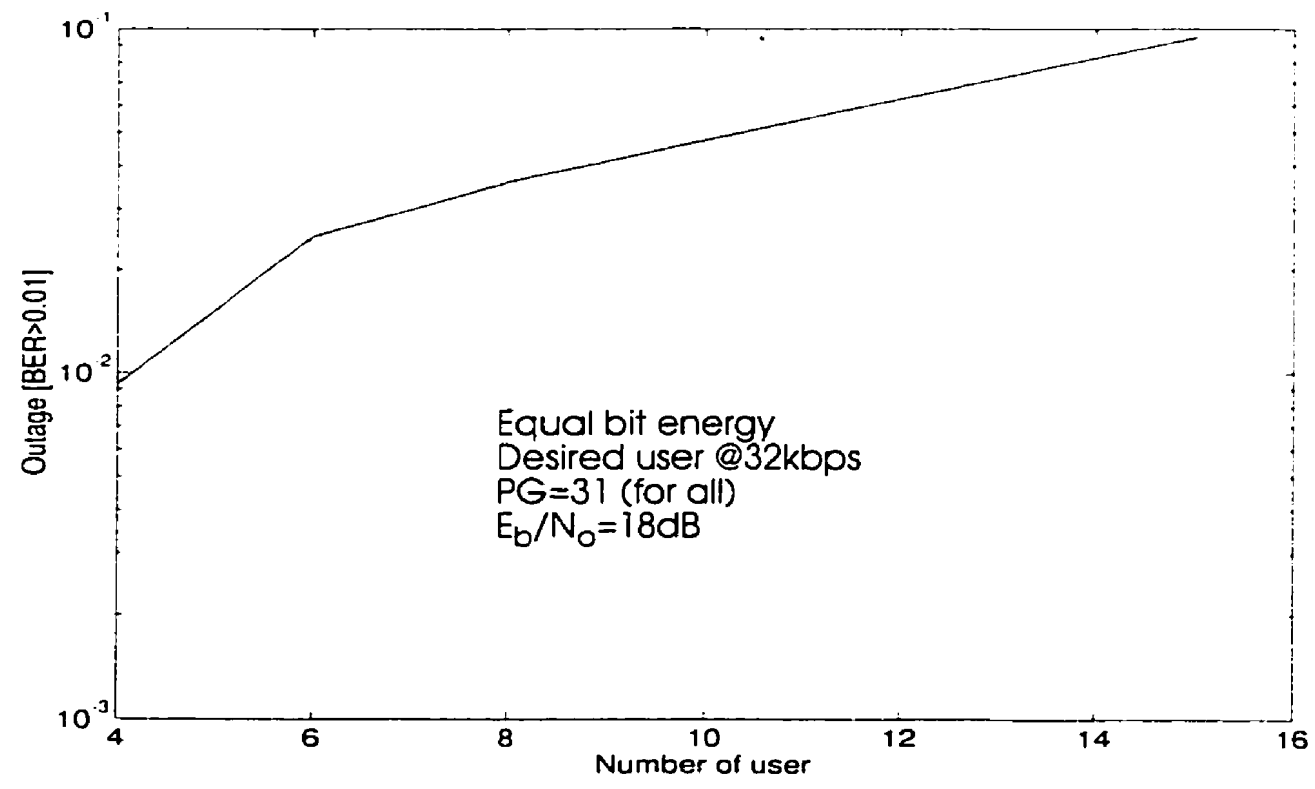

Figure 4.37: Probability of outage due to BER in scenario 1 


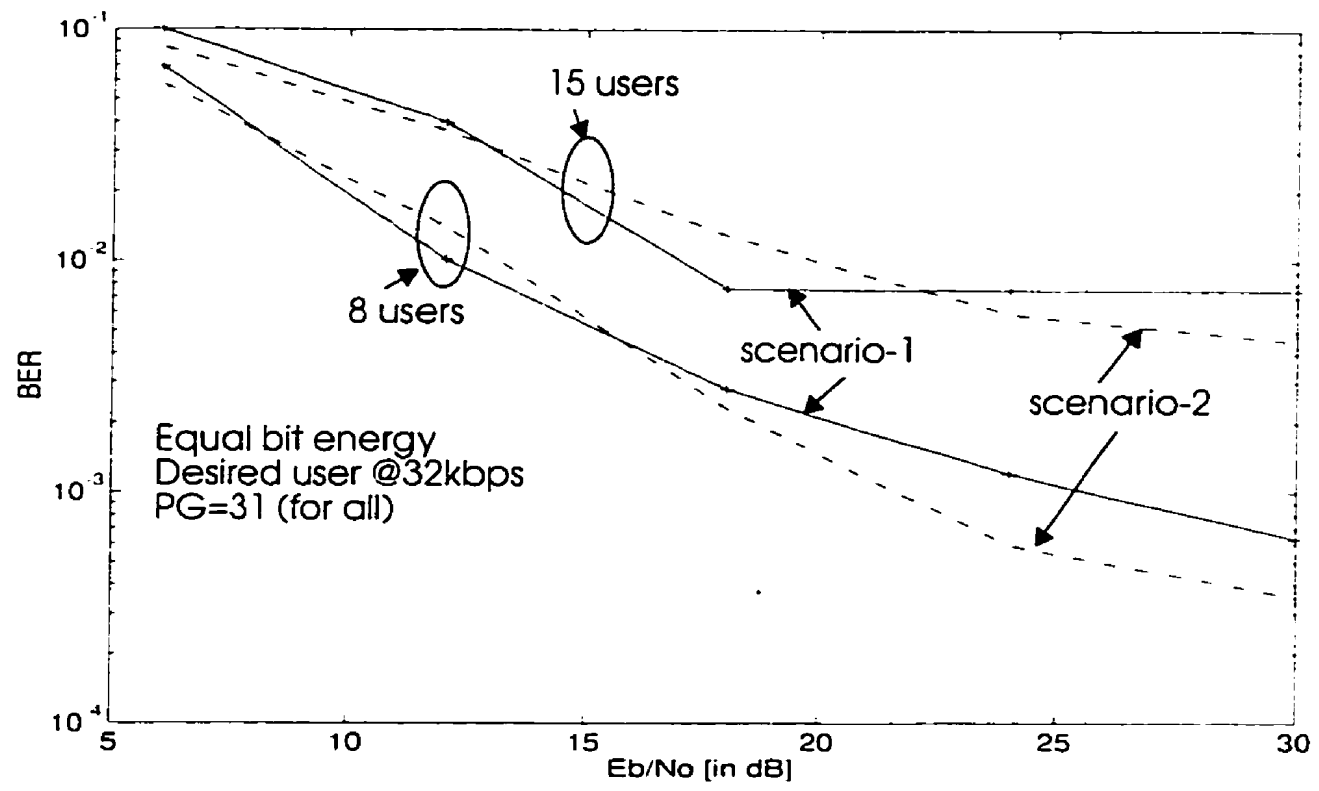

Figure 4.38: Comparison of scenario land 2 in terms of BER

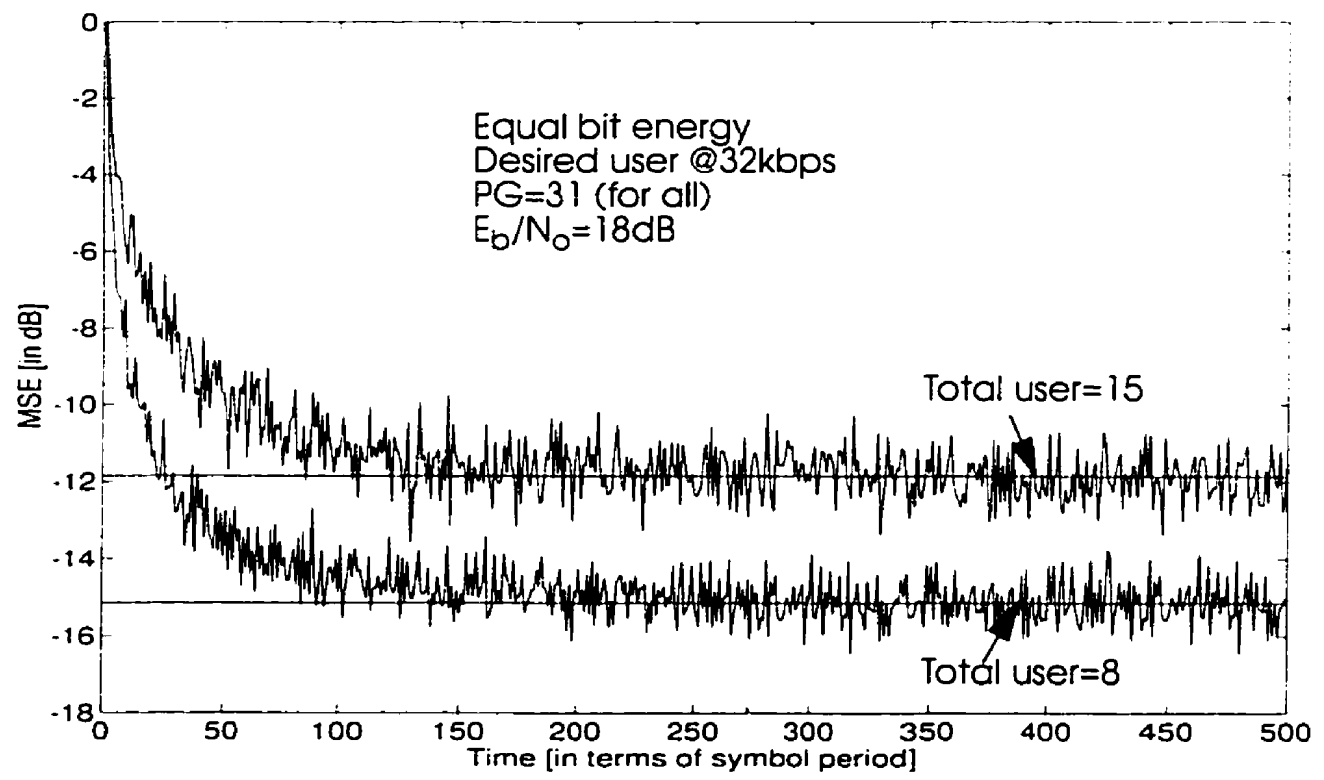

Figure 4.39: MSE performance in scenario 2 


\subsubsection{Desired user at L-data rate}

As stated before, this scenario presents the case in which the desired user is transmitting at $16 \mathrm{kbps}$ and interference is coming from a combination of users as shown in scenario- 3 and 4 of Table 3.1. The plots for this case are shown in Figure 4.40 to 4.45 . From the figures the expected performance degradation is observed as the number of user increases. Also according to expression (3.24), expected improved performance is observed for scenario-4 shown in Figure 4.44.

Comparing figures from section 4.3.1.2 with that of section 4.3.2.2 shows that when desired user is selected from L-data rate users, overall performance of the system remains statistically the same for the specified combination of users. Although. expression (3.20) and (3.24) suggests that performance in two cases (equal bit energy and equal average power) will depend on users' combination. 


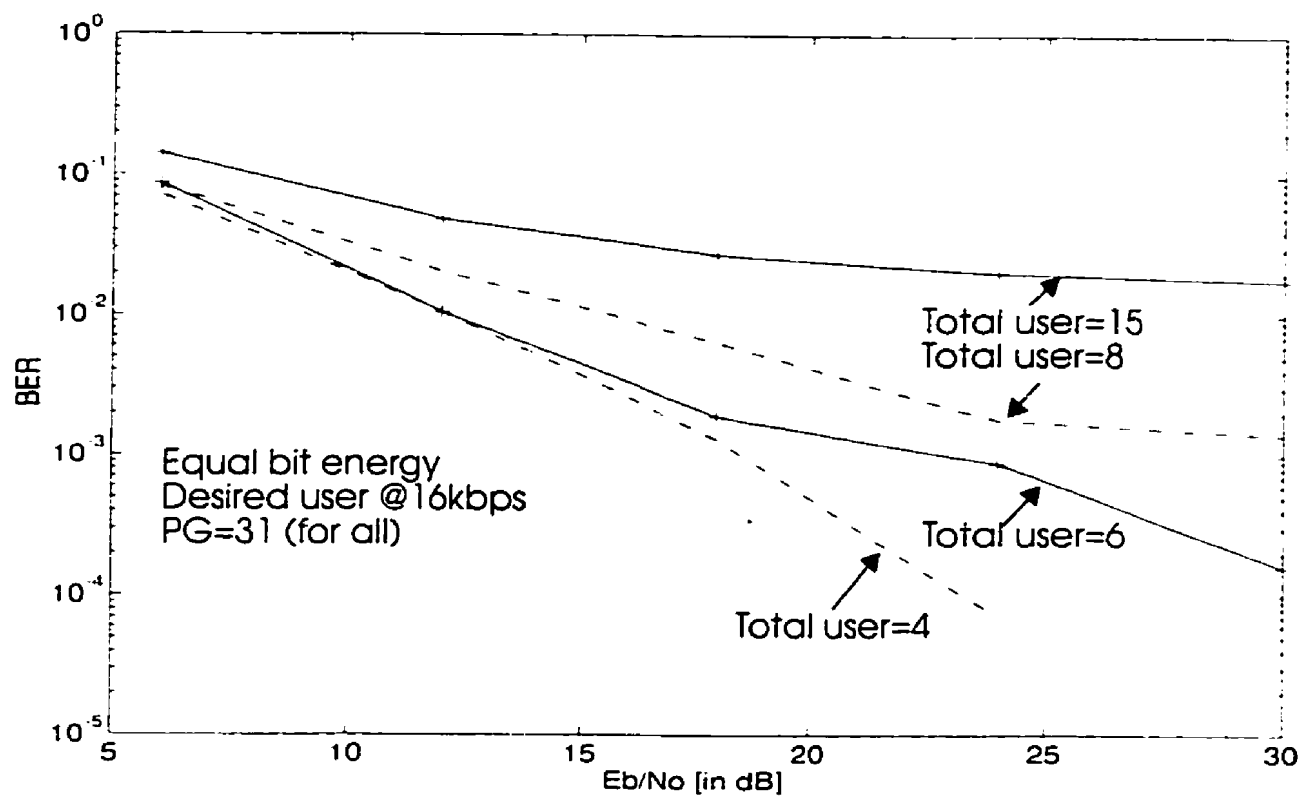

Figure 4.40: BER performance in scenario 3

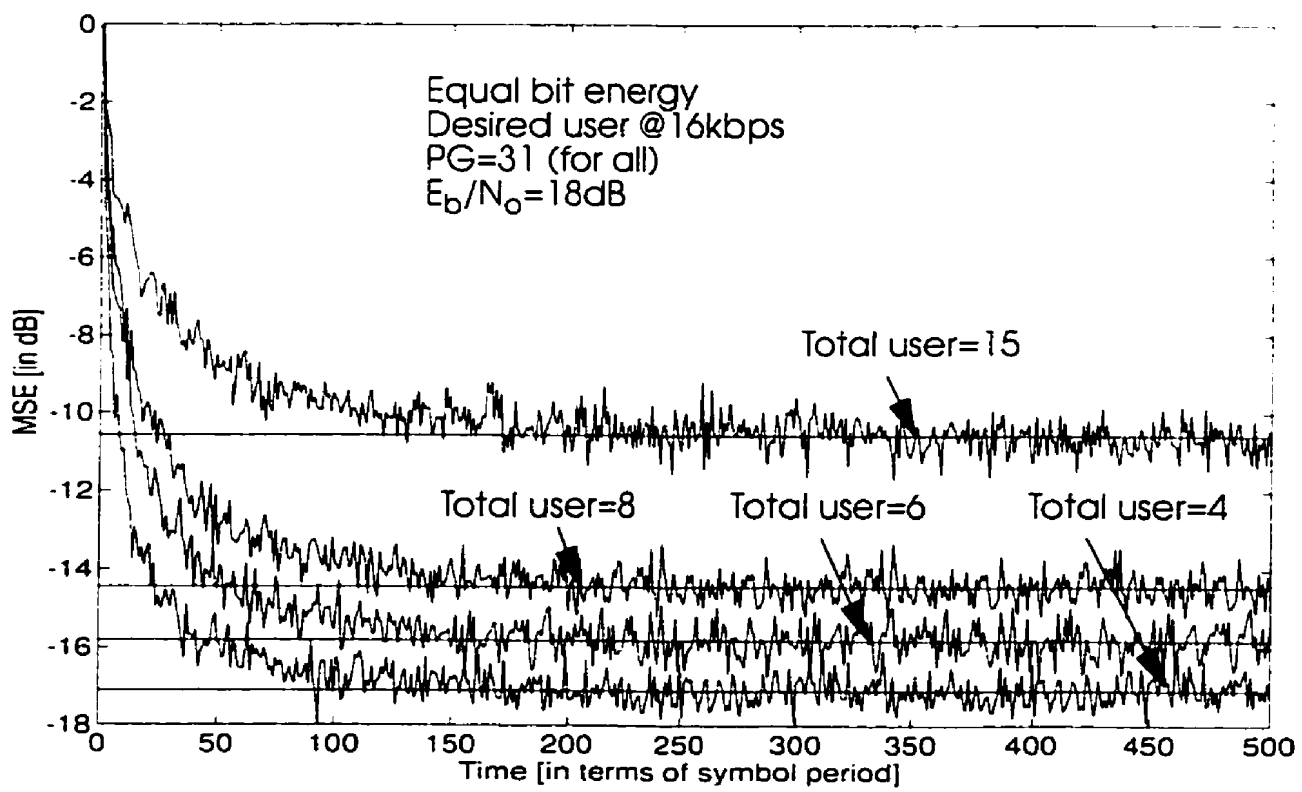

Figure 4.41: MSE performance in scenario 3 


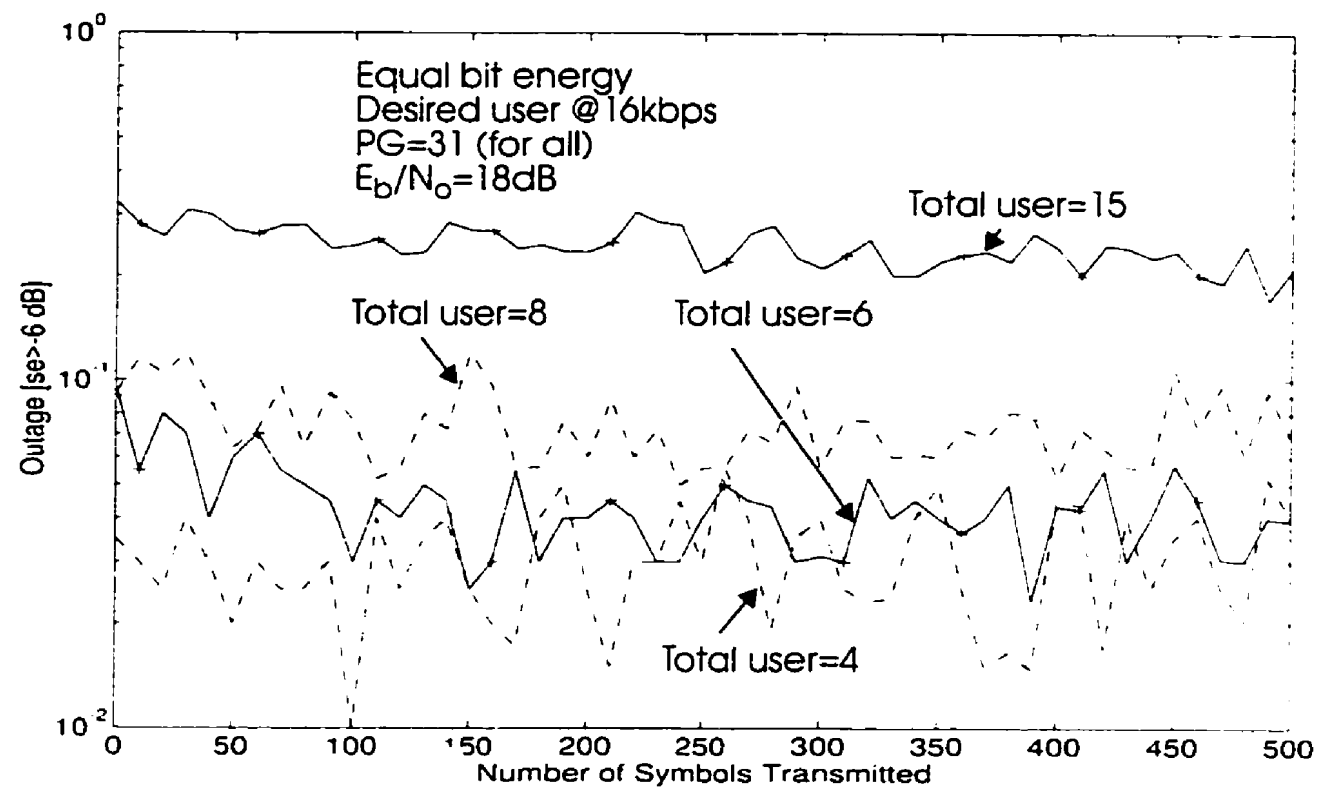

Figure 4.42: Probability of outage due to MSE in scenario 3

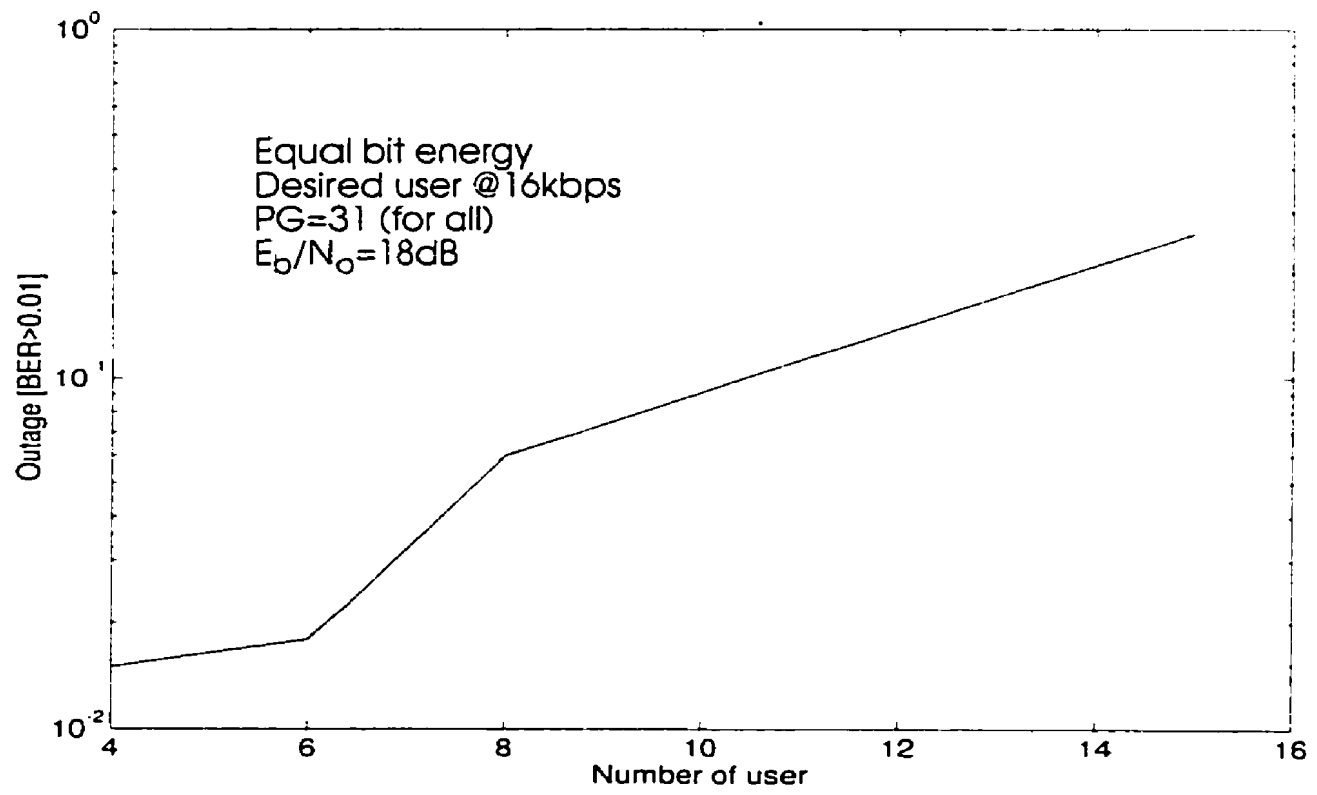

Figure 4.43: Probability of outage due to BER in scenario 3 


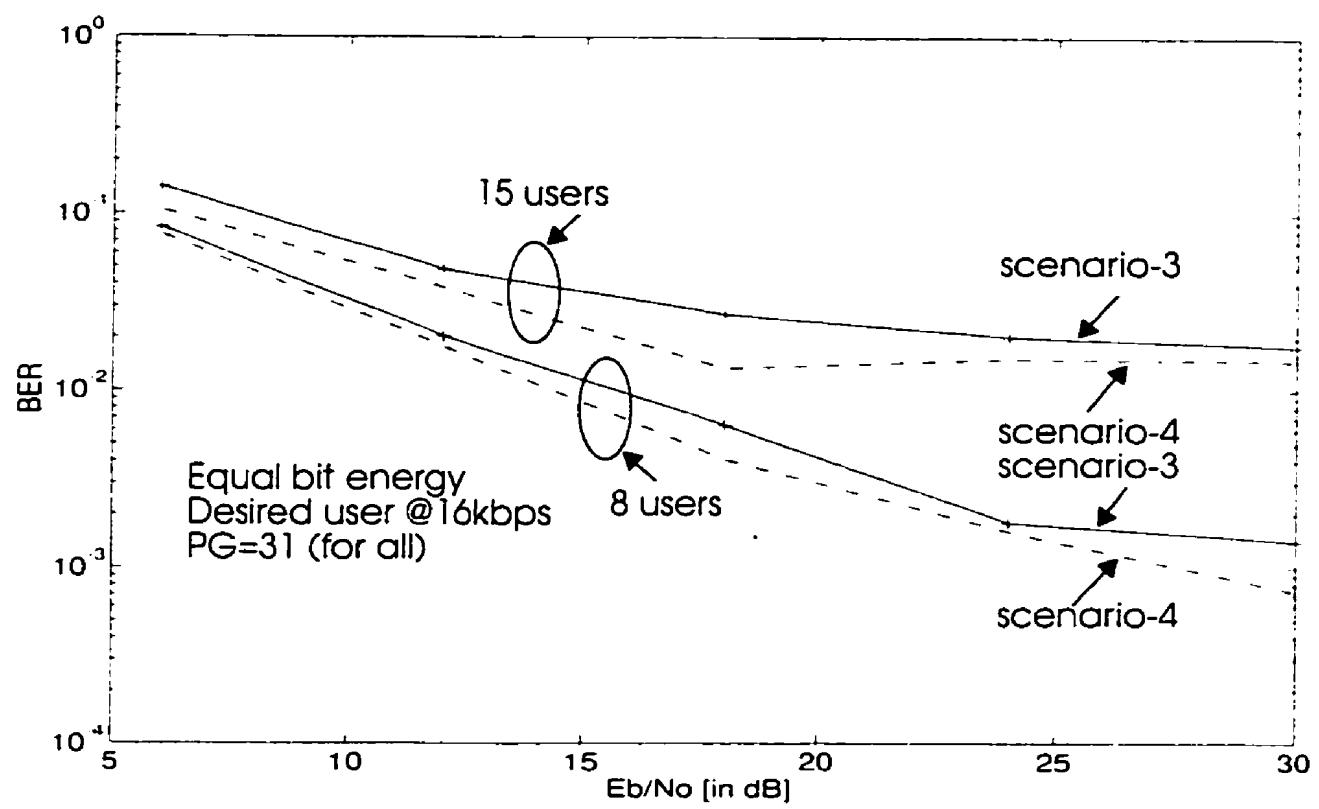

Figure 4.44: Comparison of scenario 3 and 4 in terms of BER

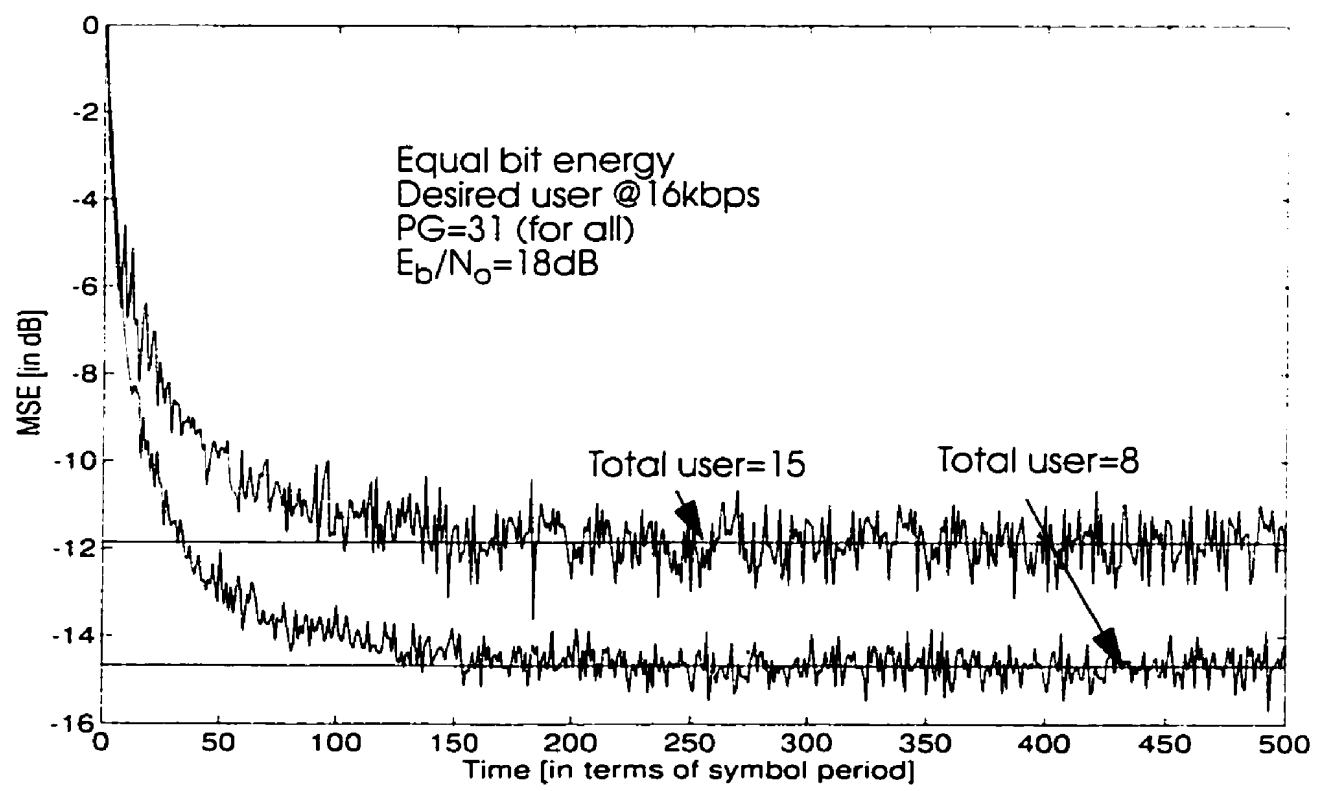

Figure 4.45: MSE performance of scenario 4 


\subsubsection{Desired user at Voice rate}

This scenario presents the case in which the desired user is transmitting at $8 k b p s$ and interference is coming from a combination of users as shown in scenario-5 and 6 of Table 3.1. The plots for this case are shown in Figure 4.46 to 4.51 . Figures in this case also shows the expected performance degradation as the number of user increases. Also according to expression (3.25), nominal improved performance is observed for scenario-6. which is shown in Figure 4.50.

Comparing figures from section 4.3.1.2 with that of section 4.3.2.2 shows that when desired user is selected from voice rate users, overall performance of the system degrades in equal bit energy case. This is just the opposite circumstance of $\mathrm{H}$-data rate user. explained in section 4.3.2.1.

As explained in section 3.6 (case-II), for same combination of users, desired user at $\mathrm{H}$-data rate may perform better than that of desired user when selected from voice or L-data rate. The simulated result also agrees with this argument. This is shown in Figure 4.52 ( for 15 user case) and 4.53 (for 8 user case) in scenario 1.3 and 5 .

Comparison of Figure 4.32 and 4.33 with Figure 4.52 and 4.53 shows that for equal bit energy and equal average power case. the performance of the system alters. but their operating region is almost same. Therefore. it may be mentioned that performance in two cases are inversely related and depends on users' combination. 


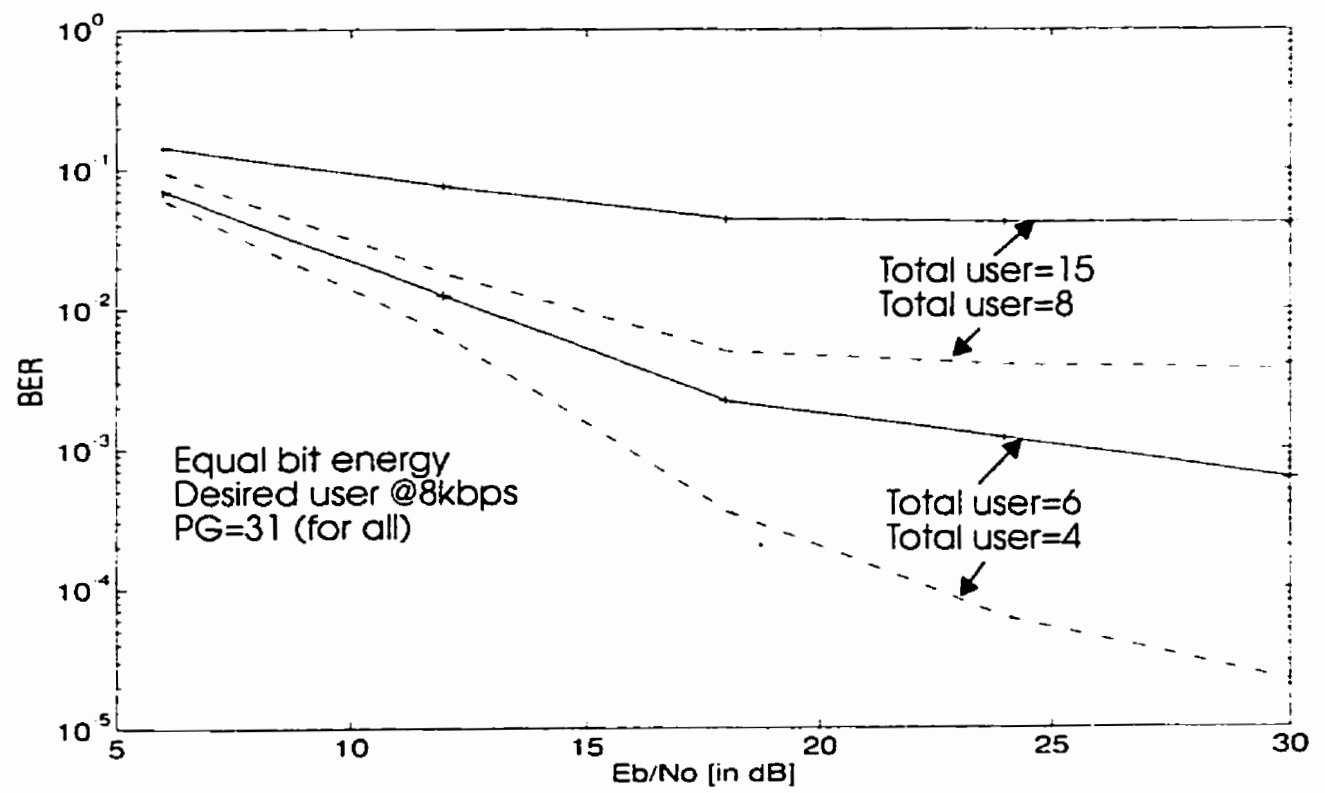

Figure 4.46: BER performance in scenario 5

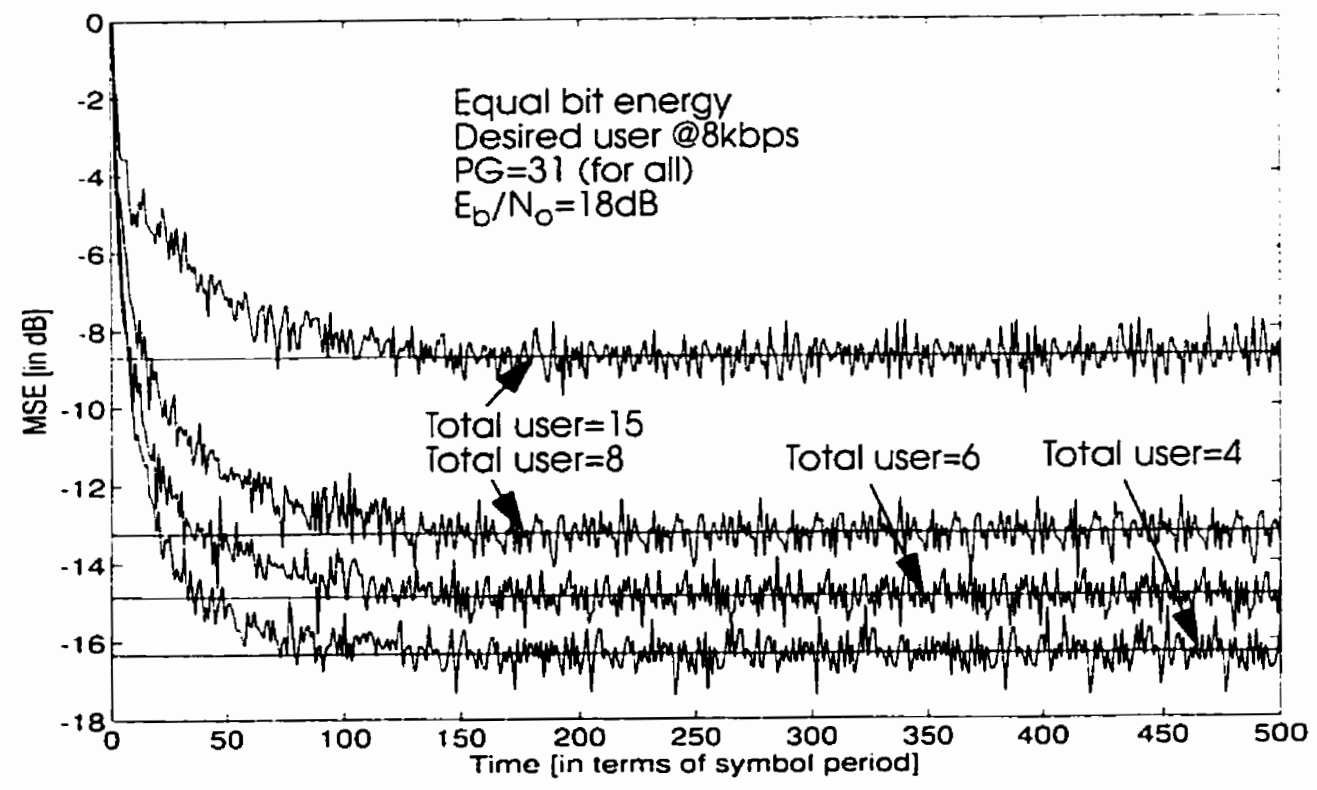

Figure 4.47: MSE performance in scenario 5 


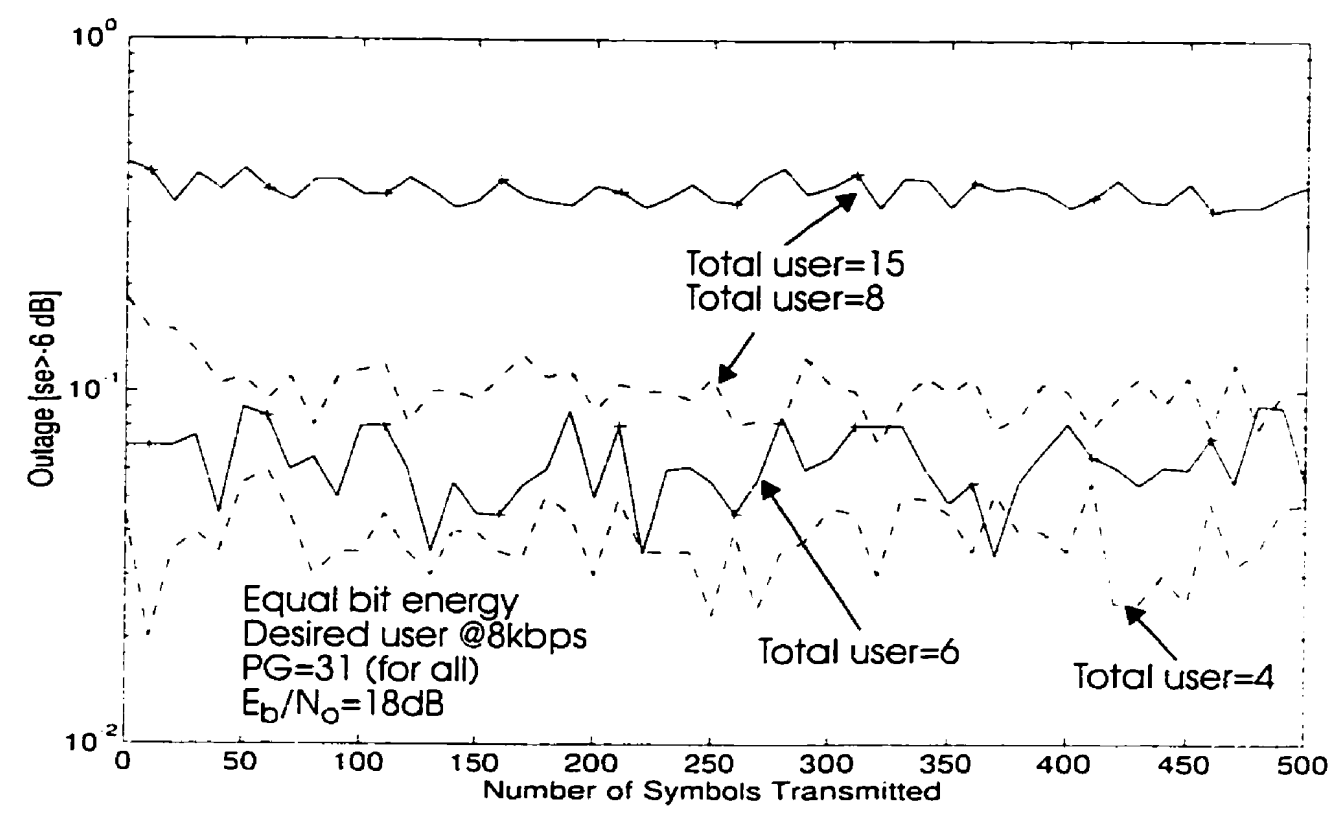

Figure 4.48: Probability of outage due to MSE in scenario 5

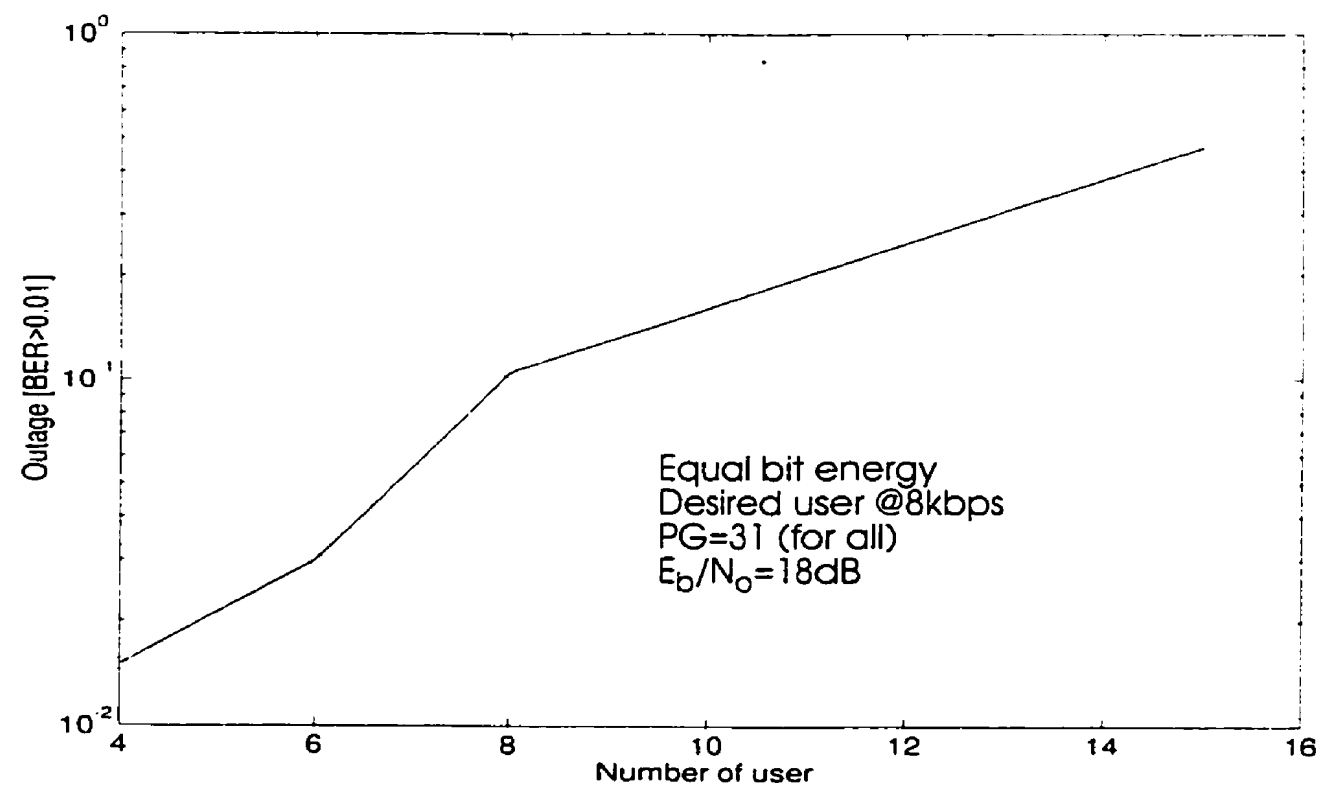

Figure 4.49: Probability of outage due to BER in scenario 5 


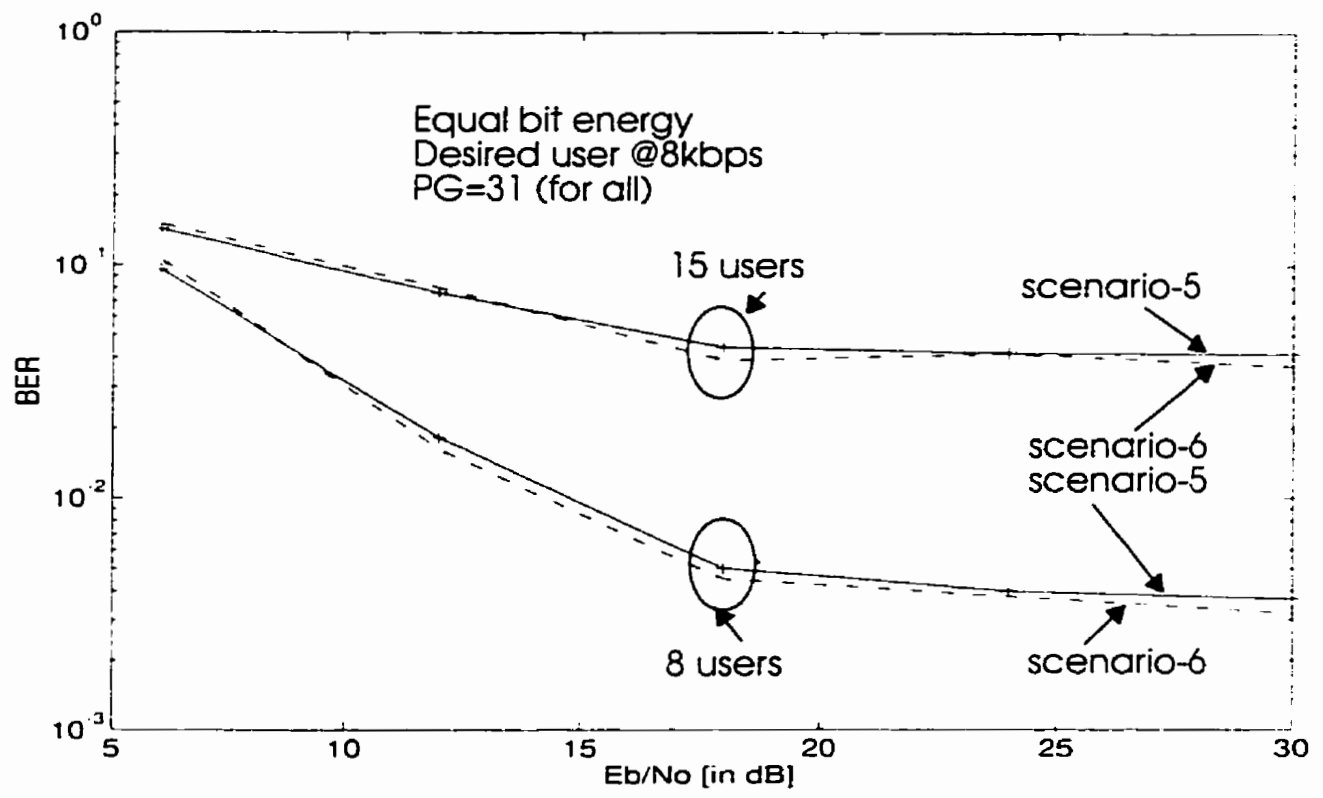

Figure 4.50: Comparison of scenario 5 and 6 in terms of BER

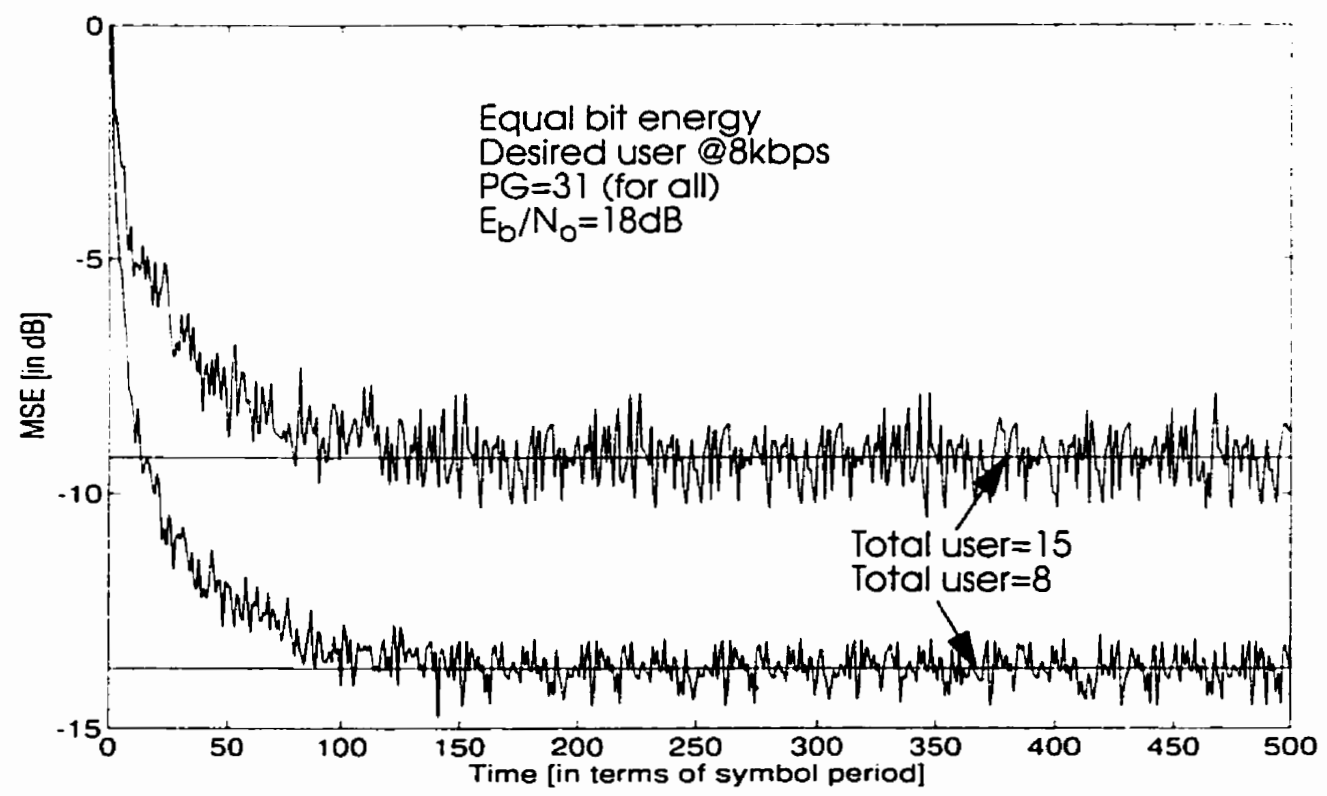

Figure 4.51: MSE performance of scenario 6 


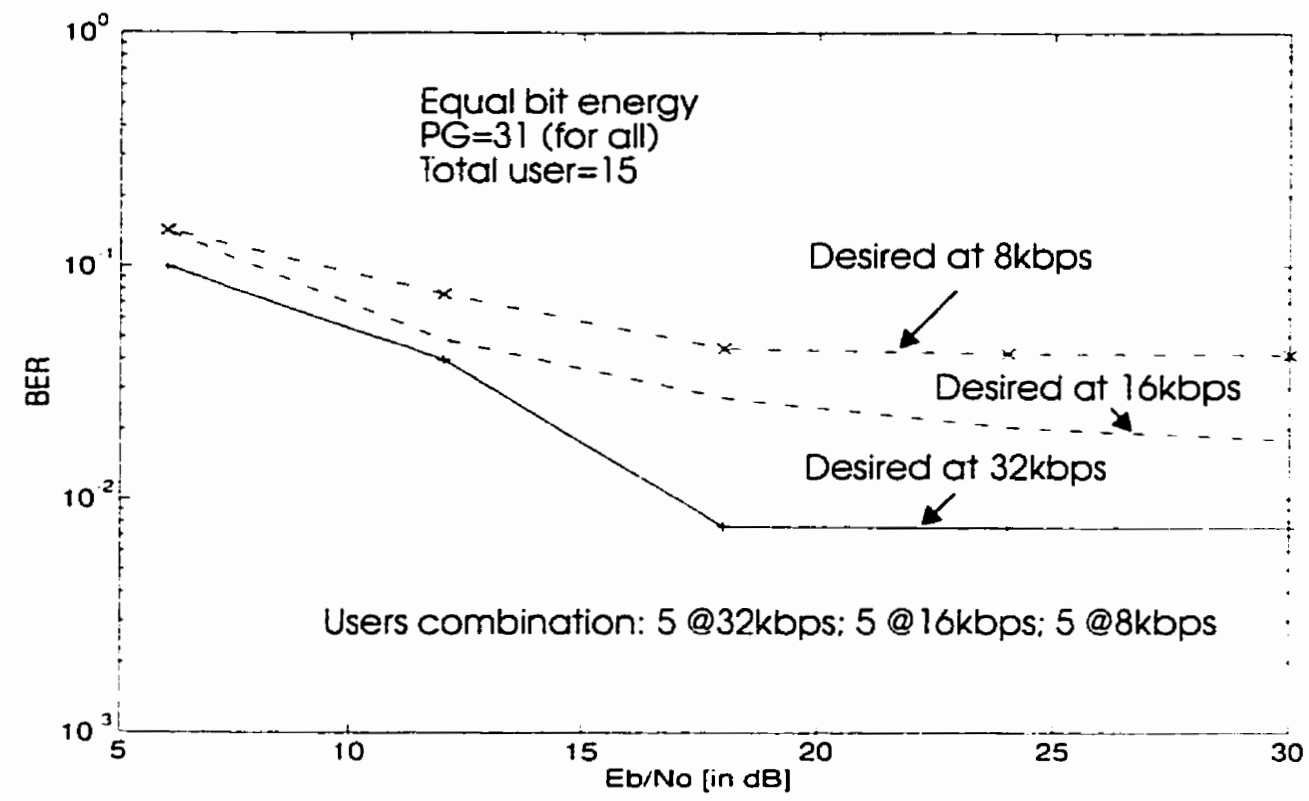

Figure 4.52: Comparison of 15 user case

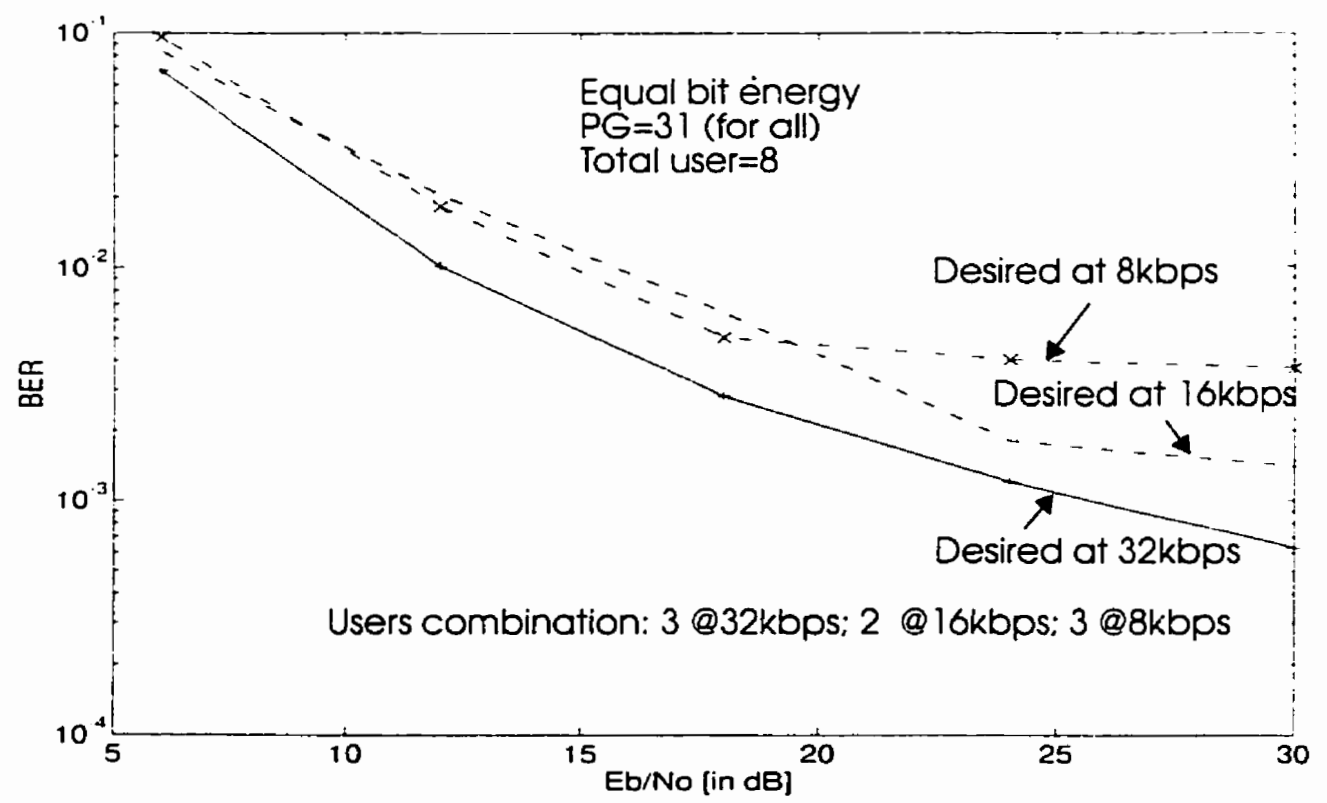

Figure 4.53: Comparison of 8 user case 


\subsection{Results for F_BW case}

Table 3.2 shows several simulation scenarios for $F \_B W$ case. Since $F \_B W$ case is simulated for the comparison purpose only. therefore. only $\mathrm{H}$-data rate and voice rate user will be considered as the desired user. This will give the upper and lower bound performance of the system. As earlier, the system is simulated under identical operating condition (only difference is the strategy taken to accommodate variable data rate) while the average power of users' are same. Here equal bit energy case is not considered as the expected performance is opposite of equal average power case (section 3.6).

\subsubsection{Equal Average Power for each user}

\subsubsection{Desired user at $\mathrm{H}$-data rate $(P G=15)$}

This scenario presents the case in which the desired user's PG is 15 . i.e. desired user is chosen from $\mathrm{H}$-data rate users. Plots for this case are shown in Figure 4.54 to 4.57. Comparing with Figure 4.8,4.9,4.10 and 4.1 1 shows that significant improvement is achieved for $V_{-} B W$ case under same operating condition.

\subsubsection{Desired user at Voice rate $(\mathrm{PG}=63)$}

This scenario presents the case in which the desired user's PG is 63. i.e. desired user is chosen from voice rate users. Plots for this case are shown in Figure 4.58 to 4.61. Since same average power criteria is taken into consideration, therefore low data rate users will have advantage over high data rate users, as explained in section 3.6. The simulation results in F_BW case also confirms this statement as shown from Figure 4.54 and 4.58. Significant improvement is again noticeable while comparing with Figure 4.24. 4.25. 4.26 and 4.27 for V_BW case under same operating condition. 


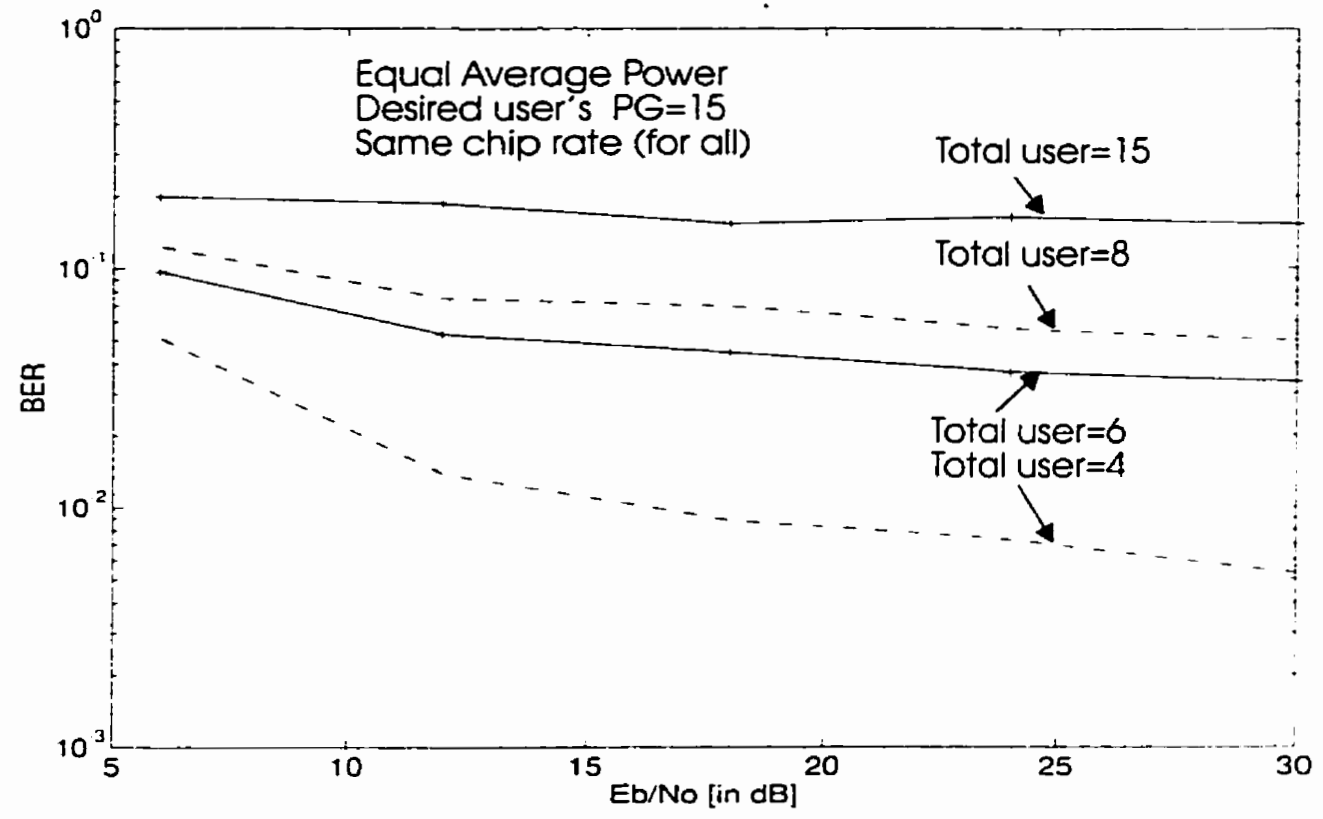

Figure 4.54: $B E R$ performance when desired user is with $P G=15$

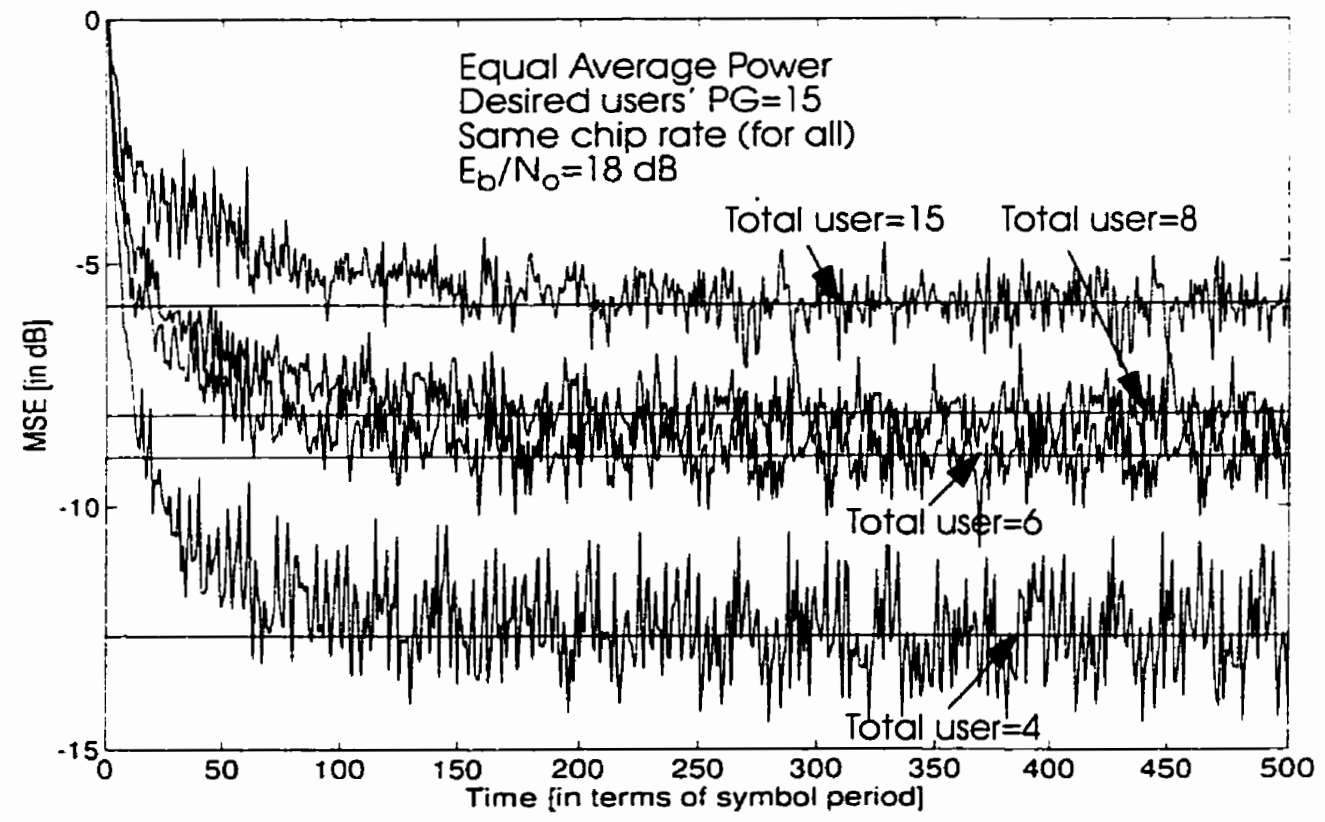

Figure 4.55: MSE performance when desired user is with $P G=15$ 


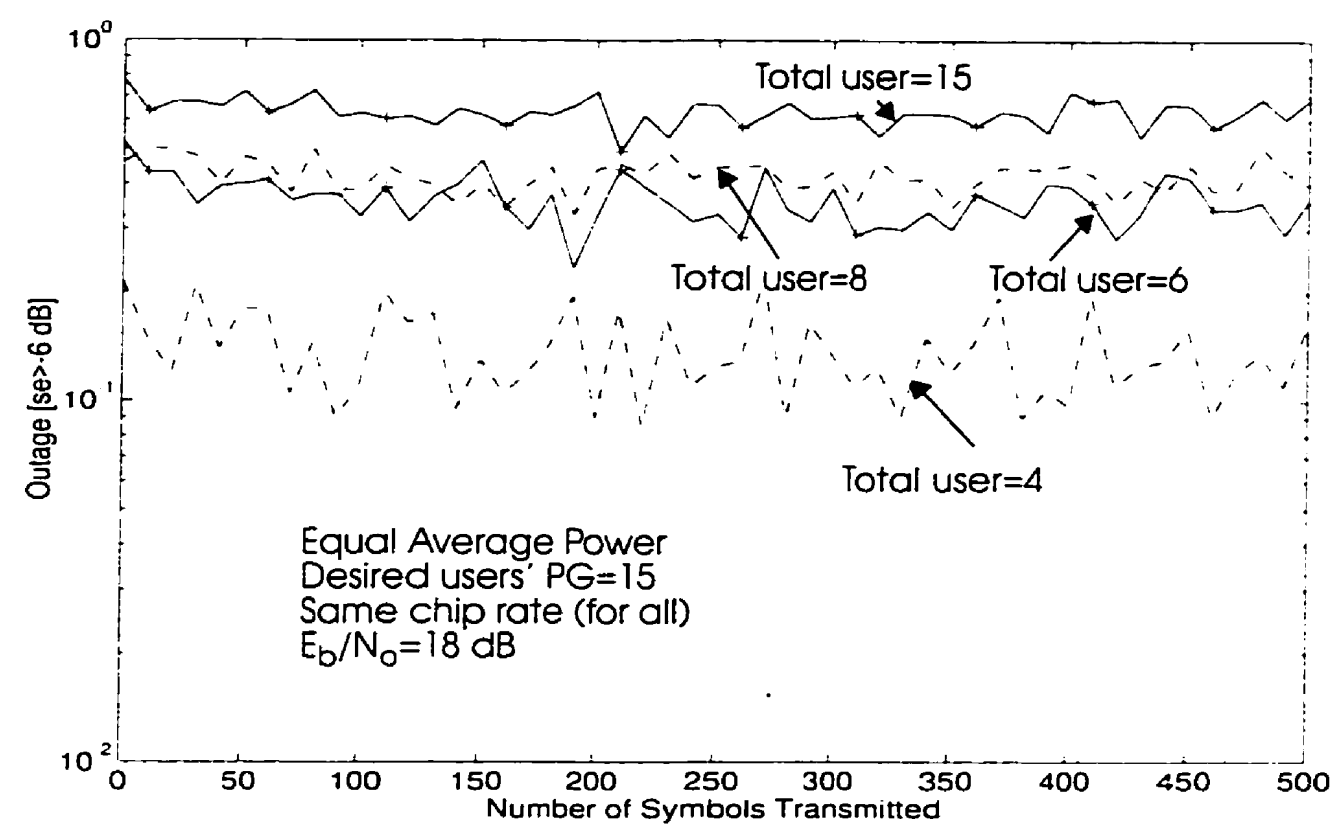

Figure 4.56: $M S E$ outage when desired user is with $P G=15$

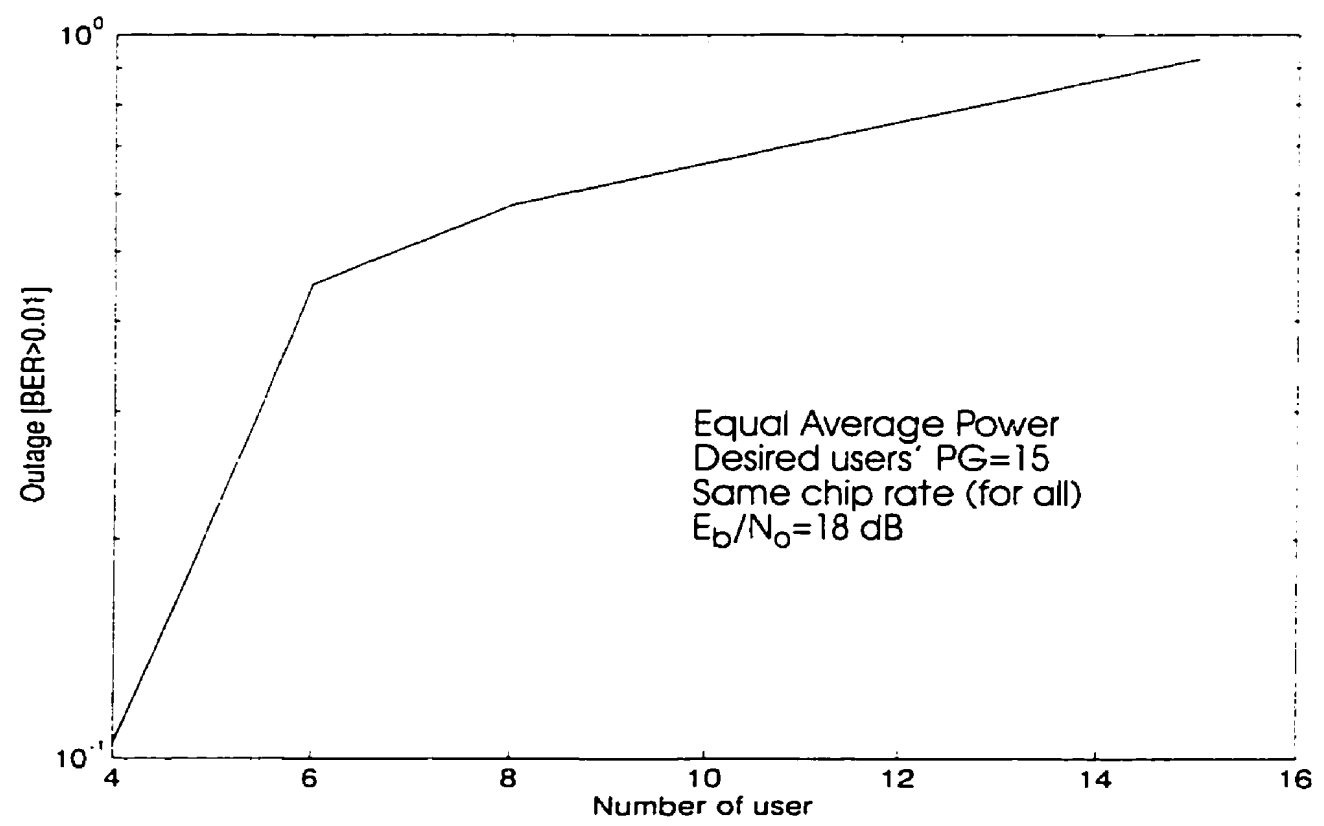

Figure 4.57: $B E R$ outage when desired user is with $P G=15$ 


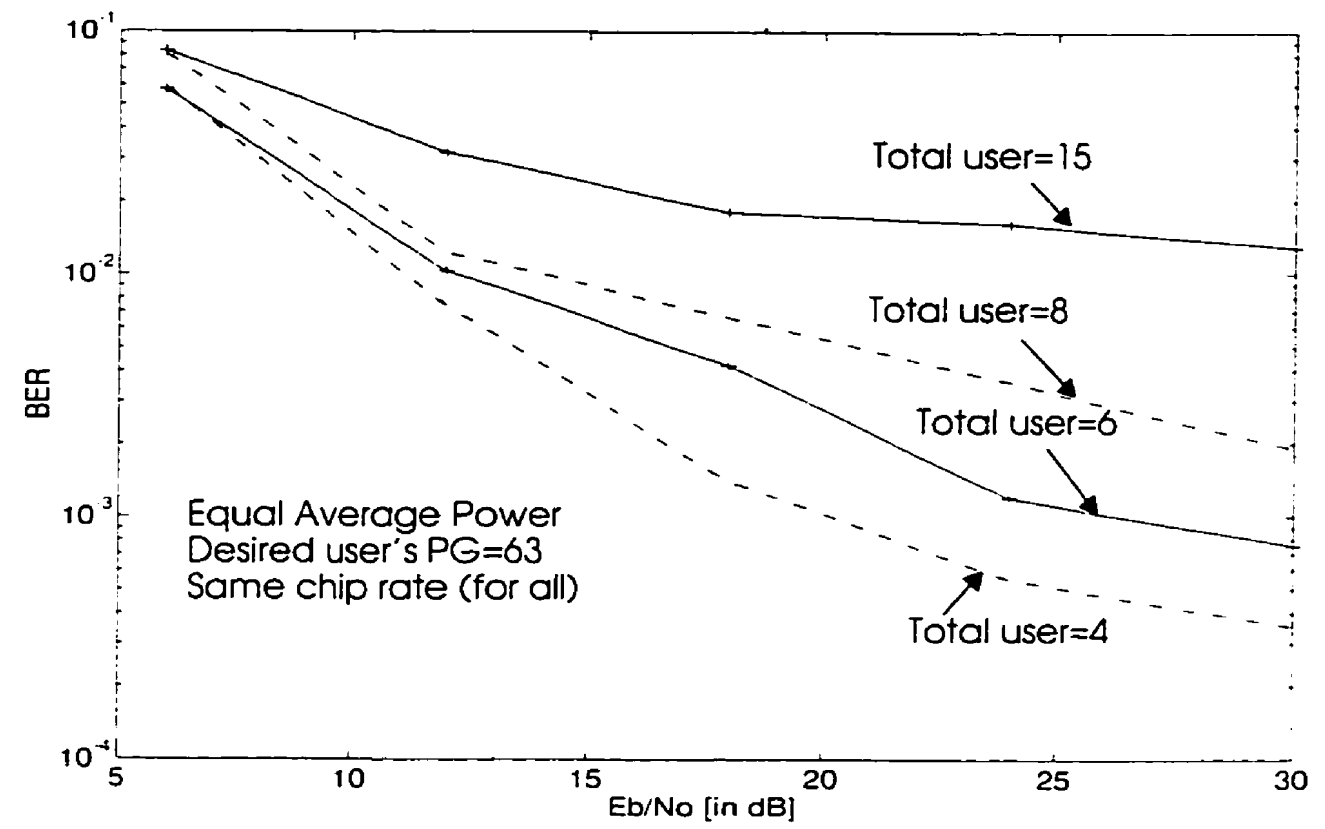

Figure 4.58: $B E R$ performance when desired user is with $P G=63$

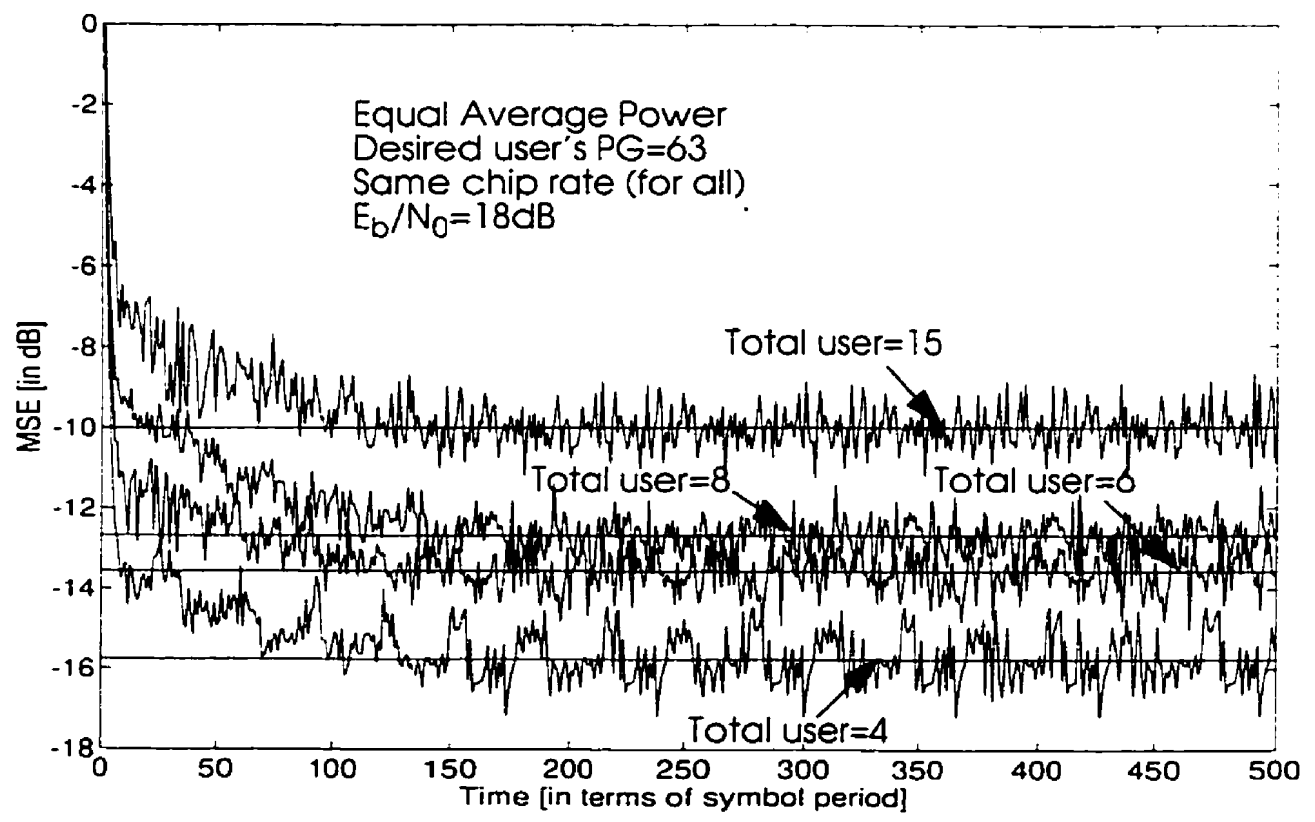

Figure 4.59: MSE performance when desired user is with $P G=63$ 


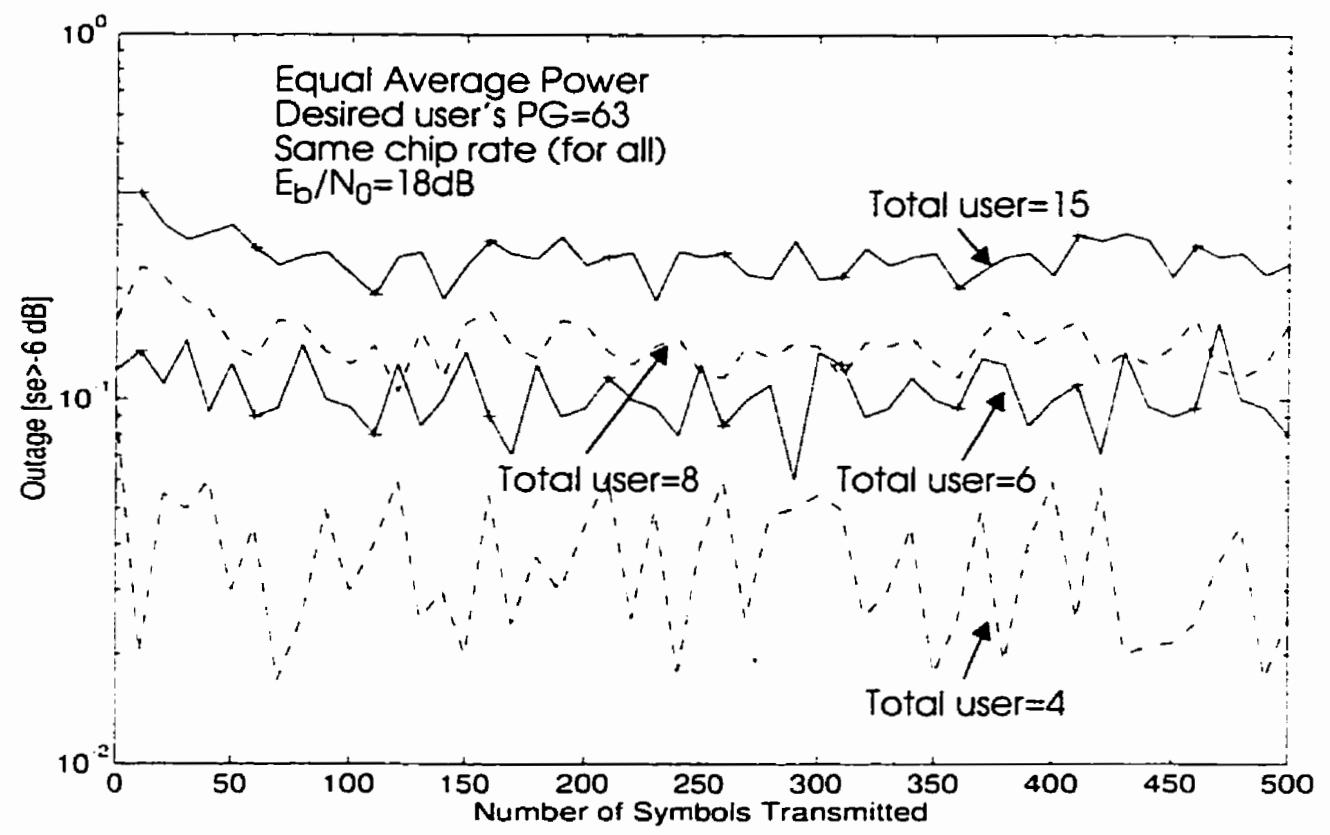

Figure 4.60: $M S E$ outage when desired user is with $P G=63$

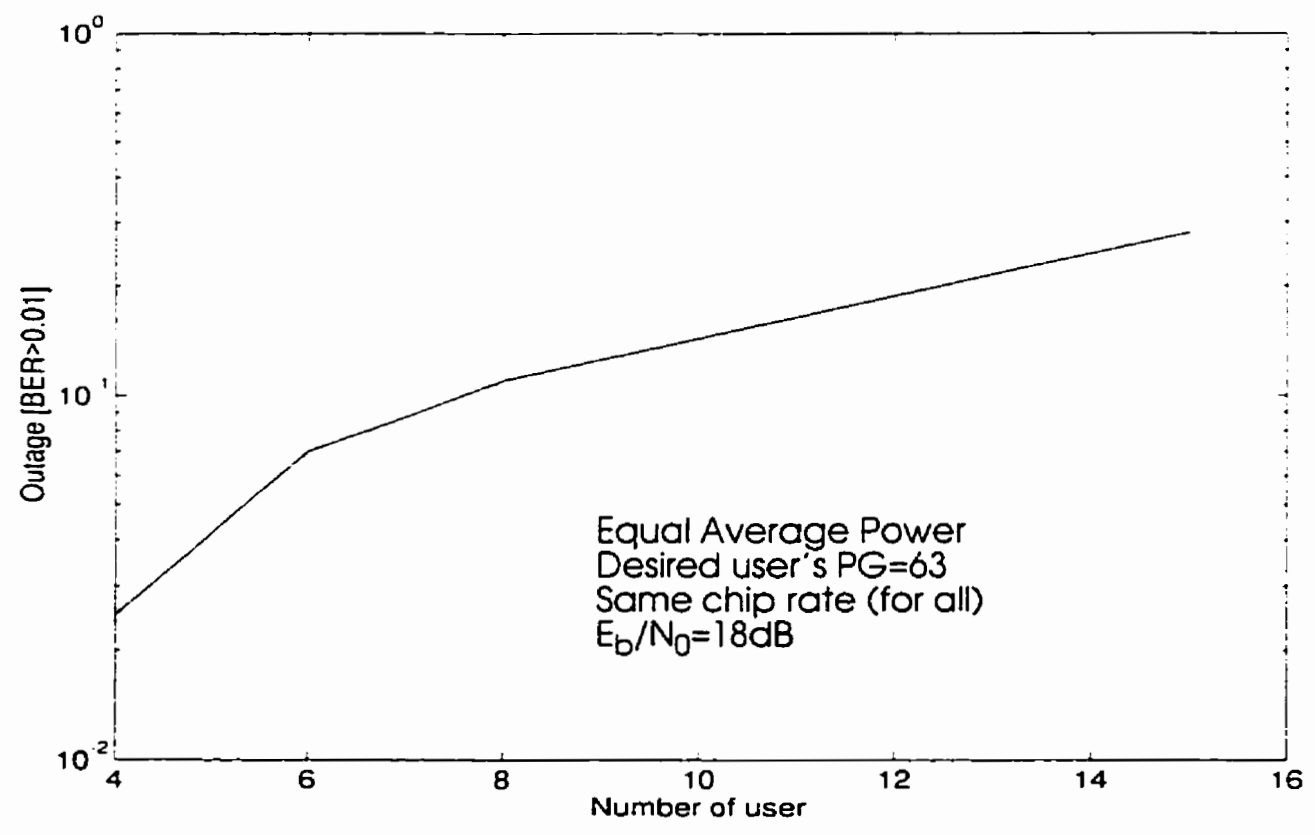

Figure 4.61: $B E R$ outage when desired user is with $P G=63$ 
Reason for improved performance in V_BW case as opposed to $\mathrm{F} \_\mathrm{BW}$ case could be due to difference in BW. In F_BW case, interference BW looking by an user (due to other users) is same. In other words, since the transmitted signal bandwidth is same for all user. therefore, entire signal spectrum of any specific user is masked by undesired users signal spectrum of same power. This raises the overall noise floor (taking interference as the dominant factor) whose height is directly related to the number of users in the system. Therefore, processing gain associated to different data rate user dominates the performance of the system.

On the other hand. in V_BW case, different data rate users occupy different bandwidths. Hence, users with different bandwidths see different interference spectra. For example. though the signal spectrum of a voice rate user is completely masked by H-data and L-data rate users, but interference power experienced from low and high data rate users are less in the equal power case (section 3.6). On the other hand, interference experienced by $\mathrm{H}$-data rate users is non-white. Thus in that case, an adaptive DFE equalizer will attempt to whiten the non-white noise as well as to suppress ISI and interference. The exact amount of improvement can be evaluated comparing the transmitted signal BW and the associated processing gain.

Work done in [58] considered $E_{C} / N_{0}=12 d B$, which means $E_{h} / N_{0} \cong 27 d B$ when user is with $\mathrm{PG}=32$ and $E_{h} / N_{0} \cong 24 d B$, with $\mathrm{PG}=16$ for all cases. Two different data rate scenario at average power control platform is considered. It is found that when desired user is at high data rate and all the interferers are at low data rate (half that of desired user). MSE for 11 user is approximately $-20 d B$ and BER is little over $10^{-3}$. Apparently, it is inconsis- 
tence with the result shown in Figure 4.54 and 4.55. But as explained in section 3.6 case-I. reduction of data rate of interferers with respect to desired user has the effect of significant performance degradation. For example, an addition of one interferer having data rate of one-fourth of that of desired user means interferers bit energy is four times higher than that of desired user and inadvertently introduce more distortion to the performance. Since three different data rates (half and one-fourth of desired user's rate) are considered, therefore. degraded performance in Figure 4.54 and 4.55 is expected to be comparable to [58]. The other reason is that in [58], the Hadamard code is used to spread the signal. which is orthogonal and has zero crosscorrelation property. But Gold code has finite crosscorrelation and contribute to raise up the noise floor. This also means that the overall height of noise floor is directly dependent on the number of users in the system. Therefore. in similar scenario, for 15 user (say). additional interference is higher if the data is spreaded using Gold code than that of Hadamard code. Hence, the overall performance degradation seen in Figure 4.54 and 4.55 is expected. Taking scenario explained in section 4.4.1 as a benchmark, significant amount of performance improvement is achieved when variable bandwidth of the transmitted signal is exploited. Similar reasoning is applicable when desired user is chosen from voice rate users (figure 4.58 and 4.59 ).

\subsection{Imperfect Average Power Control}

Imperfections in power control lead to poor performance and lower system capacity. Whenever the received power of the users are dissimilar the interference power at the output of the matched filter increases proportionally to the crosscorrelation between the spreading code of the user with that of the desired user and the performance of the system degrades. This is referred to as the near-far problem. 


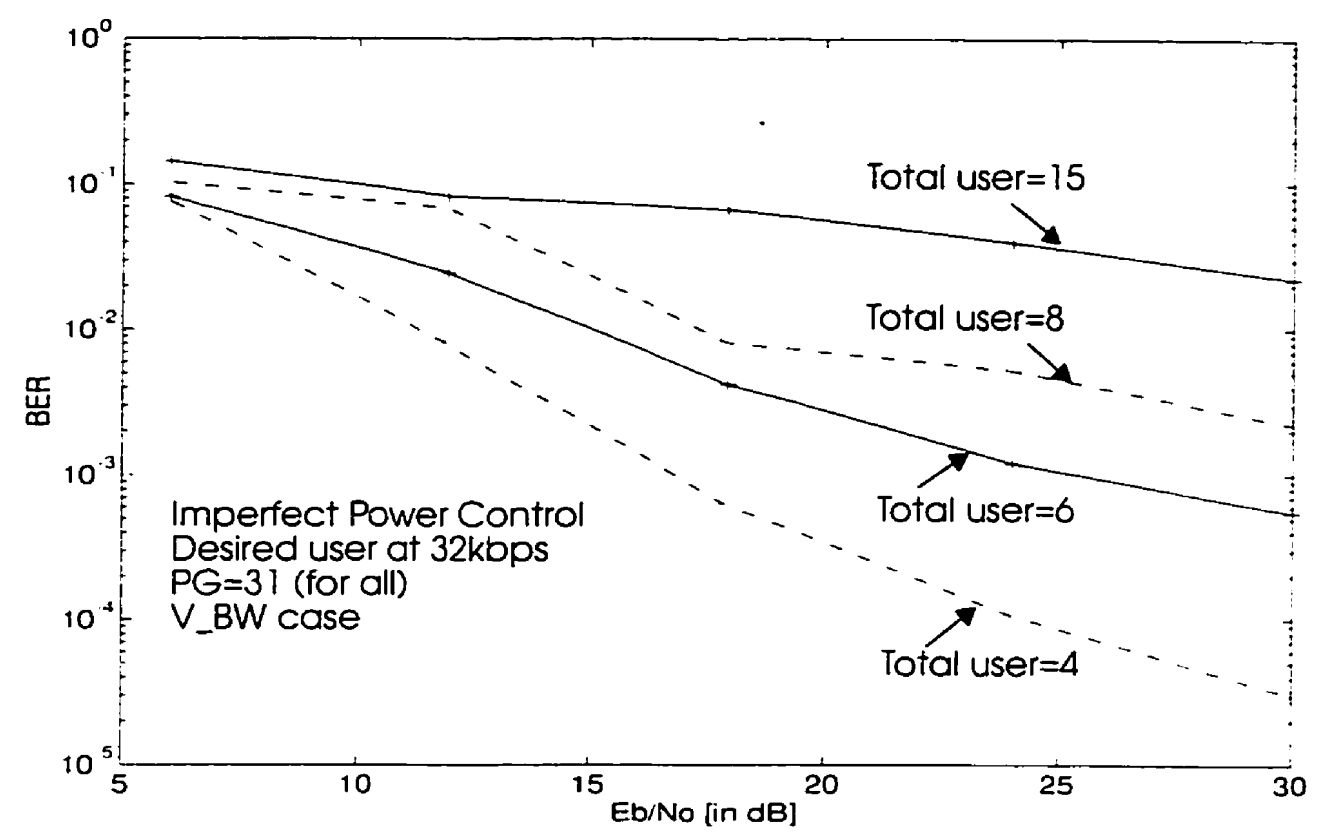

Figure 4.62: BER performance in scenario 1 (Imperfect average power control)

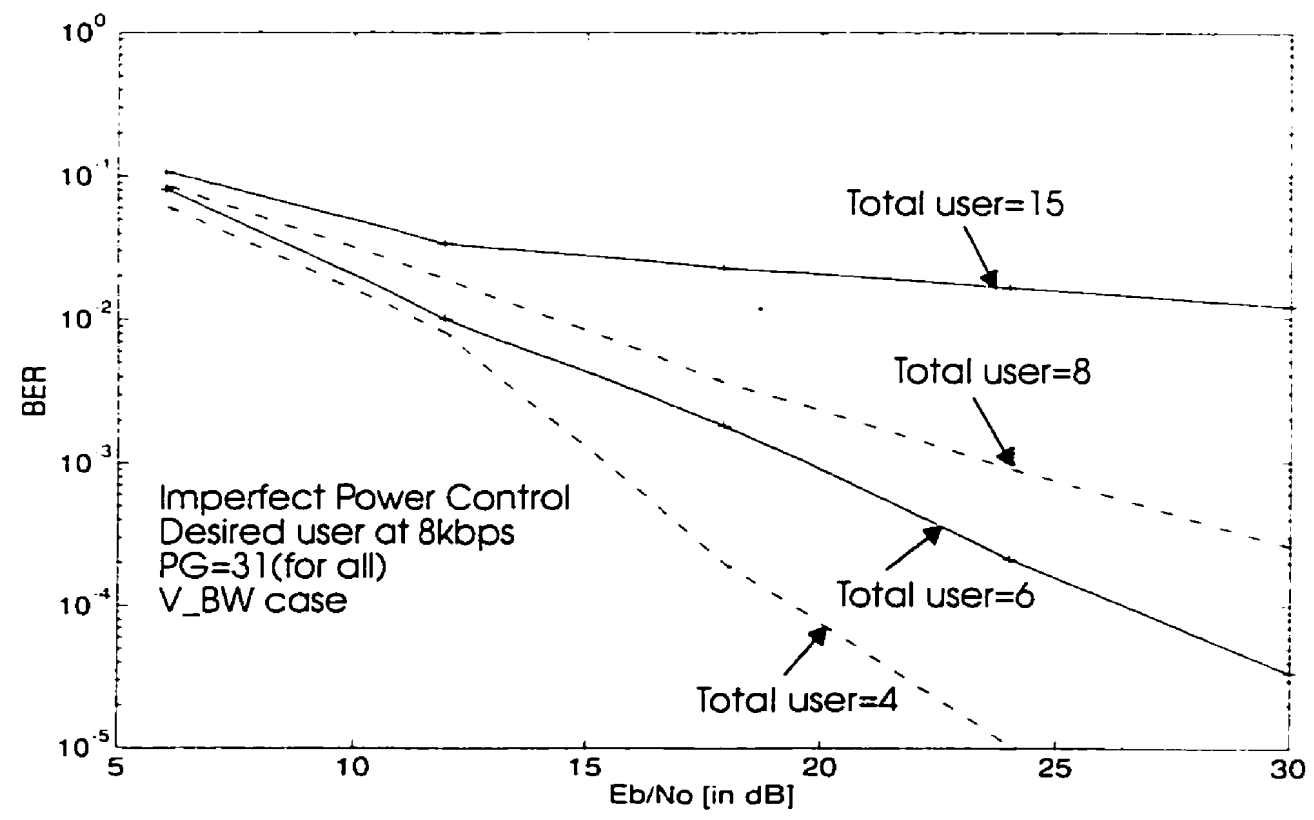

Figure 4.63: BER performance in scenario 5 (Imperfect average power control) 
The near-far problem is not an inherent property of the CDMA system rather it is a shortcoming of the receiver structure. In the previous section, all users are assumed to transmit with the same average power which is a loose form of power control known as the average power control. Conventional CDMA requires strict power control which is very hard to maintain in mobile environment. Therefore. there is a need for a receiver which is near-far resistant. In this section the chip spaced DFE is demonstrated when the average power of the users is not same. The simulation experiment is performed when the average power of interfering users differ $-5 d B$ to $+5 d B$ relative to the average power of the desired user. The power level of interfering users is assumed to be uniformly distributed around the power level of the desired user. The near-far resistance properties are investigated for scenario-1 and scenario-5 only. BER performance for these two scenarios are shown in Figure 4.62 and 4.63. Comparing these with Figure 4.8 and 4.24 respectively, shows that performance degradation in imperfect power control scenario is nominal. 


\section{Chapter 5 \\ Conclusion}

\subsection{Discussion}

This research has demonstrated the feasibility and performance advantage of a multirate CDMA cellular system taking into account the cyclostationary nature of interference. The main motivation behind this research was the intense attention and development towards implementation of PCS.

An overview of the multiuser multirate CDMA system in Rayleigh faded channel is given in chapter 3. SIR in a multiuser multirate CDMA system was also discussed. For the experiment BPSK modulation scheme and three different data rates were considered. RLS algorithm was used to update the tap weights of a chip spaced decision feedback equalizer. Among the power allocation schemes the candidate strategies are- equal average power control. equal bit energy and imperfect power control. Different scenarios that can emerge in a multirate CDMA system are considered and used for the simulation experiments.

All the experimental results are presented and discussed in chapter 4 . Before performing the simulation experiments. the receiver parameters were evaluated and the system was calibrated against the analytical expression to gain confidence level. To develop the simulated results, several combinations of users were selected. Each time one user with a specific data rate was selected as the desired user and simulation experiments were performed for $E_{b} / N_{0}$ varying from $6 d B$ to $30 d B$. The effect of interference while the interferes were at different data rate are studied. It was found that the performance of the system is depen- 
dent on specific combination of users. While the average power of users are same, any increase in number of low data rate users degrades the system performance. And low data rate users have the advantage over high data rate users in terms of BER under same operating condition. This is because, in equal average power scenario. performance is dominated by $E_{h} / N_{0}$. On the other hand, when $E_{b} / N_{0}$ is same for all users, then performance is dominated by signal power and high data rate users get advantage over low data rate users.

To accommodate different data rates, two strategies are considered (explained in section $3.2)$, but emphasis were given in variable bandwidth case, where, $P G=31$ was considered. This is because, in V_BW case, the bandwidth of interference also varies accordingly, which can be exploited for improved performance. To exploit this nature of the signal. a chip spaced decision feedback equalizer was used. In the simulation experiments spectral redundancy associated with bit sequence was considered and that with carrier frequency was avoided. To have a view on the performance of two strategies. simulated scenarios (table 3.1 and 3.2) are repeated with the results superimposed on them (table 5.1 and 5.2). In this case, BER performance at $E_{h} / N_{O}=24 d B$ is considered, since $E_{h} / N_{0}>24 d B$ does not show any significant improvement in performance for number of users $\geq 8$. From Table 5.1 and 5.2. it is found that the V_BW case performs better than that of the F_BW case. under the same operating condition.

As seen in Table 5.2, voice rate users have advantage over $\mathrm{H}$-data rate users (equal power case). It should be noted here that the equal $E_{b}$ case in $\mathrm{F}_{-} \mathrm{BW}$ scenario is not considered. This is because the expected performance in equal $E_{b}$ case is opposite of equal power case (as explained in section 3.6), under the same operating condition. This means that in equal 
$E_{h}$ case. $\mathrm{H}$-data rate users have the advantage over voice rate users. Therefore. BER for the desired user with $P G=15$ is expected to be relatively lower than that shown in Table 5.2.

Table 5.1: Results in V_BW case

\begin{tabular}{|c|c|c|c|c|c|c|c|}
\hline \multirow{2}{*}{$\begin{array}{c}\text { Desired } \\
\text { users' } \\
\text { Info }\end{array}$} & \multirow{2}{*}{$\begin{array}{l}\text { Sce- } \\
\text { nario }\end{array}$} & \multicolumn{3}{|c|}{ \# of Interfering users } & \multirow{2}{*}{$\begin{array}{l}\text { Total } \\
\text { user }\end{array}$} & \multicolumn{2}{|c|}{ BER at $24 d B$} \\
\hline & & $\begin{array}{c}\mathrm{H}- \\
\text { data }\end{array}$ & $\begin{array}{l}\text { L- } \\
\text { data }\end{array}$ & Voice & & Equal $P$ & Equal $E_{h}$ \\
\hline \multirow{6}{*}{$\begin{array}{c}\text { H-data } \\
\mathrm{R}_{\mathrm{b}}=32 \\
\text { kbps }\end{array}$} & \multirow[t]{4}{*}{1} & 4 & 5 & 5 & 15 & $1.69 \times 10^{-2}$ & $7.5 \times 10^{-3}$ \\
\hline & & 2 & 2 & 3 & 8 & $2.2 \times 10^{-3}$ & $1.2 \times 10^{-3}$ \\
\hline & & 1 & 2 & 2 & 6 & $8.2 \times 10^{-4}$ & $3.88 \times 10^{-4}$ \\
\hline & & 1 & 1 & 1 & 4 & $6.3 \times 10^{-5}$ & $2.15 \times 10^{-5}$ \\
\hline & \multirow[t]{2}{*}{2} & 1 & 9 & 4 & 15 & $1.8 \times 10^{-2}$ & $5.9 \times 10^{-3}$ \\
\hline & & 1 & 4 & 2 & 8 & $1.8 \times 10^{-3}$ & $5.88 \times 10^{-4}$ \\
\hline \multirow{6}{*}{$\begin{array}{c}\text { L-data } \\
\mathrm{R}_{\mathrm{b}}=16 \\
\text { kbps }\end{array}$} & \multirow[t]{4}{*}{3} & 5 & 4 & 5 & 15 & $1.4 \times 10^{-2}$ & $2.03 \times 10^{-2}$ \\
\hline & & 3 & 1 & 3 & 8 & $7.16 \times 10^{-4}$ & $1.8 \times 10^{-3}$ \\
\hline & & 2 & 1 & 2 & 6 & $1.19 \times 10^{-4}$ & $8.83 \times 10^{-4}$ \\
\hline & & 1 & 1 & 1 & 4 & $1.12 \times 10^{-5}$ & $7.22 \times 10^{-5}$ \\
\hline & \multirow[t]{2}{*}{4} & 2 & 8 & 4 & 15 & $1.6 \times 10^{-2}$ & $1.5 \times 10^{-2}$ \\
\hline & & 2 & 3 & 2 & 8 & $3.9 \times 10^{-4}$ & $1.6 \times 10^{-3}$ \\
\hline \multirow{6}{*}{$\begin{array}{l}\text { Voice } \\
\mathrm{R}_{\mathrm{b}}=8 \\
\text { kbps }\end{array}$} & \multirow[t]{4}{*}{5} & 5 & 5 & 4 & 15 & $8.6 \times 10^{-3}$ & $4.2 \times 10^{-2}$ \\
\hline & & 3 & 2 & 2 & 8 & $5.4 \times 10^{-4}$ & $4.0 \times 10^{-3}$ \\
\hline & & 2 & 2 & 1 & 6 & $1.6 \times 10^{-4}$ & $1.2 \times 10^{-3}$ \\
\hline & & 1 & 1 & 1 & 4 & $\mathrm{xx}$ & $6.19 \times 10^{-5}$ \\
\hline & \multirow[t]{2}{*}{6} & 2 & 9 & 3 & 15 & $1.16 \times 10^{-2}$ & $4.19 \times 10^{-2}$ \\
\hline & & 2 & 4 & 1 & 8 & $6.24 \times 10^{-4}$ & $3.8 \times 10^{-3}$ \\
\hline
\end{tabular}


On the other hand. BER for the desired user with $P G=63$ is expected to be relatively higher than that shown in Table 5.2. To obtain these differences in performance requires additional experiments.

All the simulation experiments were performed in Rayleigh faded channel. because it gives the worse case scenario. Performance of the DFE in a near-far scenario were also presented in the last section of chapter 4. It was seen that the performance of DFE does not vary much when the average power of the interfering users varies in a range of $-5 d B$ to $+5 d B$ with respect to the desired users power. Thus the proposed structure can even tolerate imperfect average power control. This result is important, because the main problem in current CDMA systems is that they do not perform well without power control.

Table 5.2: Results in $F \_B W$ case

\begin{tabular}{|c|c|c|c|c|c|}
\hline $\begin{array}{c}\text { Total } \\
\text { user }\end{array}$ & $\mathrm{PG}=15$ & $\mathrm{PG}=31$ & $\mathrm{PG}=63$ & Desired & $\begin{array}{c}\text { BER at } 24 d B \\
(\text { Equal } P \text { ) }\end{array}$ \\
\hline 15 & 5 & 5 & 5 & & $1.6 \times 10^{-1}$ \\
\hline 8 & 3 & 2 & 3 & \multirow{2}{*}{$\mathrm{PG}=15$} & $5.58 \times 10^{-2}$ \\
\cline { 1 - 4 } & 2 & 2 & 2 & & $3.69 \times 10^{-2}$ \\
\hline 4 & 2 & 1 & 1 & & $7.3 \times 10^{-3}$ \\
\hline \hline 15 & 5 & 5 & 5 & \multirow{4}{*}{$\mathrm{PG}=63$} & $1.6 \times 10^{-2}$ \\
\hline 8 & 3 & 2 & 3 & $3.6 \times 10^{-3}$ \\
\hline 6 & 2 & 2 & 2 & & $1.2 \times 10^{-3}$ \\
\hline 4 & 1 & 2 & 1 & & $5.51 \times 10^{-4}$ \\
\hline
\end{tabular}




\subsection{Proposals for Future Work}

In this thesis, although many scenarios were investigated, a number of issues remain unresolved and require further study. It was assumed that all the users are on the same carrier frequency. It would be interesting to see the performance in a multicarrier scenario, where the signal of different data rate users will be modulated using different carrier. In that way, spectral redundancy associated with carrier can also be exploited in addition to bit rate redundancy.

In the simulation experiments. co-channel and adjacent channel interference have not been considered. It will be worthwhile to observe the performance in the presence of co-channel and adjacent channel interference along with distance power law and shadowing effect. Also factors like coding, antenna sectorization. voice activity factor can be included for additional improvement and thus to present a more realistic system.

An expression for BER in Rayleigh faded channel in the absence of multipath. assuming interference as the additional AWGN has been derived. It will be worthwhile to derive an expression assuming interference having cyclosationary nature and incorporating three dimensional channel model (employing multipath and doppler shift). Other channel models like, Nakagami. can also be considered. 


\section{Appendix}

\section{A. Detail Mathematics for probability of bit error}

To evaluate the probability of error of any digital modulation scheme in a slow. flat fading channel, one must average the probability of error of the particular modulation in AWGN channels over the possible signal range of signal strength due to fading. In other words, the probability of error in AWGN channel is viewed as a conditional error probability, where the condition is that $\alpha$ is fixed. Hence the probability of error in slow, flat fading channels can be obtained by averaging the error in AWGN channels over the fading probability density function. In doing so, the probability of error in slow, flat fading channel can be evaluated as [59]. [60]

$$
P_{e}=\int_{0}^{x} P_{e}(X) f_{X^{(}}(x) d X
$$

where $P_{e}\left(X^{\prime}\right)$ is the probability of error for an arbitrary modulation at a specific value of signal to noise ratio $X$, and $X$ is defined as $X=\alpha^{2}\left(E_{h} / N_{0}\right)$ and $f_{X}(x)$ is the probability density function of $X$ due to the fading channel. $E_{b}$ and $N_{\emptyset}$ are constants. and the random variable $\alpha$ is used to represent amplitude values of the fading channel. with respect to $E_{h}$ / $N_{0}$.

The probability of error in AWGN channel (no multipath and fading) in the presence of interference (assuming interference as white noise). can be expressed [59] as 


$$
P_{e}=Q\left(\sqrt{\frac{1}{\frac{1}{3 N} \sum_{k=1}^{K-1} \frac{P_{k}}{P_{0}}+\frac{N_{0}}{2 E_{b}}}}\right)
$$

where $K$ is the total number of users, $N$ is the length of code, $P_{k}$ is power associated with the user $k . P_{0}$ is the desired users' power and $E_{b} / N_{0}$ is signal to noise ratio. In typical CDMA environments. communication links are interference limited, not noise limited [59]. Therefore expression (A.2) can be reduced to

$$
P_{e}=Q\left(\sqrt{\frac{1}{\frac{1}{3 N} \sum_{k=1}^{K-1} \frac{P_{k}}{P_{0}}}}\right)
$$

In fading channel. the conditional probability of error can be approximated. conditioned upon $\alpha_{k}$ and $\alpha_{0}$ as

$$
P\left(e / \alpha_{k}, \alpha_{0}\right)=Q\left(\sqrt{\frac{1}{\frac{1}{3 N} \sum_{k=1}^{K-1} \frac{\alpha^{2}{ }_{k} P_{k}}{\alpha_{0}{ }^{2} P_{0}}}}\right)
$$

For perfect power control scenario. where $P_{k}=P_{0}$ for all $k=1,2 \ldots \ldots . K$. equation (A.4) become

$$
P\left(e / \alpha_{k}, \alpha_{0}\right)=Q\left(\sqrt{\frac{1}{\frac{1}{3 N} \sum_{k=1}^{K-1} \frac{\alpha^{2}}{\alpha_{0}^{2}}}}\right)
$$

For Rayleigh fading channel $\alpha$ has a Rayleigh distribution, given by 


$$
f_{\alpha}(\alpha)=\frac{\alpha}{r^{2}} \exp \left(-\alpha^{2} / 2 r^{2}\right) \quad \alpha \geq 0
$$

Let $M=\alpha^{2}$. Therefore,

$$
F_{M}(m)=P(M \leq m)=P(\alpha \leq \sqrt{m})=F_{\alpha}(\sqrt{m})
$$

Hence,

$$
f_{. M}(m)=\frac{d}{d m} F_{\alpha}(\sqrt{m})=f_{\alpha}(\sqrt{m}) /(2 \sqrt{m})
$$

From (A.6) \& (A.8) we have

$$
f_{. M}(m)=\frac{1}{2 r^{2}} \exp \left(\frac{-m}{2 r^{2}}\right)=\frac{1}{R} \exp \left(\frac{-m}{R}\right) \quad \mathrm{m} \geq 0
$$

which shows that $M$ have a chi-square distribution with two degrees of freedom. where

$$
\Gamma=\overline{\alpha^{2}} \frac{E_{h}}{N_{0}}
$$

is the average value of signal to noise ratio at the receiver front. Substituting the value of $\alpha^{2}$ in (A.5), we get

$$
P\left(e / M_{k}, M_{0}\right)=Q\left(\sqrt{\frac{1}{\frac{1}{3 N} \sum_{k=1}^{K-1} \frac{M_{k}}{M_{0}}}}\right)
$$

Let us assume $U=M_{1}+M_{2}+\ldots \ldots \ldots \ldots \ldots \ldots+M_{k}$. where the pdf of $M$ is given in equation (A.9). The characteristic function of $M$ is

$$
\Phi_{M}(\omega)=\int_{0}^{x} f_{. M}(m) \exp (j \omega m) d m=\frac{\frac{\mathrm{l}}{R}}{\frac{1}{R}-j \omega}
$$

Assuming all $M_{k}$ 's are iid random variables, the characteristic function of $U$ is 


$$
\Phi_{C}(\omega)=\left\{\frac{\frac{1}{R}}{\frac{1}{R}-j \omega}\right\}^{K-1}
$$

Hence, the pdf of $U$ is Gamma distributed.

$$
f_{C}(u)=\frac{\frac{1}{R}\left(\frac{u}{R}\right)^{K-2} \exp \left(\frac{-u}{R}\right)}{(K-2) !}=\frac{u^{K-2} \exp \left(\frac{-u}{R}\right)}{R^{K-1}(K-2) !}
$$

Now let us define $X=U / M_{0}$ and $Y=M_{0}$, which results $U=X M_{0}$ and $M_{0}=Y$. The Jacobian of the inverse transformation is

$$
|J(x, y)|=\operatorname{det}\left[\begin{array}{cc}
\frac{\partial}{\hat{c} u} & \frac{\hat{c} u}{\hat{c} y} \\
\frac{\hat{c} m_{0}}{\hat{\partial} x} & \frac{\partial m_{0}}{\hat{\partial} y}
\end{array}\right]=\left[\begin{array}{cc}
m_{0} & 0 \\
0 & 1
\end{array}\right]=m_{0}
$$

Assuming $U$ and $M_{0}$ as independent random variable, the joint pdf of $\mathrm{X}$ and $\mathrm{Y}$ can be written as

$$
\begin{aligned}
& f_{x . y}(x, y)=f_{L, u_{n}}\left(u, m_{0}\right)|J(x, y)|_{u=x y} \\
& m_{11}=y
\end{aligned}
$$

From (A.15) and (A.16) we get the joint pdf of $X$ and $Y$ as

$$
f_{x, y}(x, y)=\frac{y(x y)^{K-2} e^{\frac{-x y}{R}} e^{\frac{-y}{R}}}{R^{K}(K-2) !}
$$

Therefore, pdf of $X$ is

$$
f_{X^{\prime}}(x)=\frac{x^{K-2}}{R^{K}(K-2) !} \int_{0}^{x} y^{K-1} e^{-y \cdot\left(\frac{x}{R}+\frac{1}{R}\right)} d y
$$

Rearranging and solving equation (A.18) we get

$$
f_{. X}(x)=\frac{(K-1) x^{K-2}}{(x+1)^{K}}
$$


Hence the average probability of error in Rayleigh faded channel is

$$
P_{e}=\int_{0}^{x} \frac{(K-1) x^{K-2}}{(x+1)^{K}} Q\left(\sqrt{\frac{3 N}{x}}\right) d x
$$

or.

$$
P_{\mathrm{e}}=\frac{1}{2} \int_{0}^{x} \frac{(K-1) x^{K-2}}{(x+1)^{K}} \operatorname{erfc}\left(\sqrt{\frac{3 N}{2 x}}\right) d x
$$




\section{References}

[1]: Khaled Ben Letaief, "Efficient Evaluation of the Error Probabilities of Spread-Spectrum Multiple-Access Communication", IEEE Transaction on Communications. Vol.45, No.2, Feb.'97, pp. 239.

[2]: F. Giannetti, "Capacity Evaluation of a Cellular CDMA System Operating in the 63$64 \mathrm{GHz}$ Band". IEEE Transactions on Vehicular Technologv, Vol.46. No.1. Feb.'97. pp. 55.

[3]: M. Benthin, Karl-Dirk Kammeyer, "Influence of Channel Estimation of a Coherent DS-CDMA System", IEEE Transactions on Vehicular Technolog: Vol.46. No.2, May.'97, pp. 262.

[4]: M. Wittmann, J. Marti, T. Kurner, "Impact of the Power Delay Profile Shape on the Bit Error Rate in Mobile Radio Systems", IEEE Transactions on Vehicular Technologr: Vol.46, No.2, May.'97, pp. 329.

[5]: N. Benvenuto, L. Tomba, "Performance Comparison of Space Diversity and Equalization Techniques for Indoor Radio", IEEE Vehicular Technologv; vol.46. No.2. May'97, pp. 358.

[6]: K.L. Yeung, S. Nanda, "Channel Management in Microcell / Macrocell Cellular Radio Systems", IEEE Transactions on Vehicular Technology, Vol.45. No.4, Nov.'96, pp. 601.

[7]: A. Masoomzadeh-Fard, S. Pasupathy, "Nonlinear Equalization in Multipath Fading Channels with Noncoherent Demodulation", IEEE Journal on Selected areas on Communications", vol.14, Apr.'96. pp. 512.

[8]: J.E. Padgett, C.G. Gunter, T. Hattory, "Overview of Wireless Personal Communications", IEEE Communication Magazine. Jan '95, pp. 28.

[9]: B.S. Abrams, A.E. Zeger, T.E. Jones, "Efficiently Structured CDMA Receiver with Near-Far Immunity", IEEE Transactions on Vehicular Technology; Vol.44, No.1. Feb.'95. pp. 1.

[10]: D.M. Grieco, "The Capacity Achievable with a Broadband CDMA Microcell Underlay to an Existing Cellular Macrosystem", IEEE Journal on Selected Areas on Communications, vol.12, No.12, May '94, pp. 744.

[11]: M. Frullone, G. Riva, P. Grazioso, M. Missiroli, "Comparisons of multiple Access Schemes for Personal Communication Systems in a Mixed Cellular Environment". IEEE Transactions on Vehicular Technology, vol.43, No.1, Feb.'94, pp. 99. 
[12]: M. Abdulrahman, A.U.H. Sheikh, D.D. Falconer. "Decision Feedback Equalization for CDMA in Indoor Wireless Communications", IEEE Journal on Selected Areas on Communications, vol.12, No.4, May '94, pp. 698.

[13]: Ozan K. Tonguz, "Cellular CDMA Networks Impaired by Rayleigh Fading: System Performance with Power Control," IEEE Transaction on Vehicular technologi: vol.43, No.3. Aug.94, pp. 515.

[14]: H. Hashemi, "The Indoor Radio Propagation Channel", Proc. of the IEEE, vol.81. No.7, Jul.'93, pp. 943.

[15]: C.M. Simmonds, M.A. Beach, "Network Planning Aspects of DS-CDMA with Particular Emphasis on Soft Handoff". Proc. 43rd IEEE Vehicular Technology Conference 93 . pp. 846.

[16]: P.B. Rapajic, B.S. Vucetic. "A Linear Adaptive Fractionally Spaced Single User Receiver for Asynchronous CDMA Systems", IEEE International Sympositum on Information Theory '93, pp. 45.

[17]: S.K. Wilson, J.M. Cioff, "Equalization Techniques for Direct Sequence Code Division Multiple Access Systems in Multipath Channels", IEEE International Sympositu on Information Theory '93. pp. 47.

[18]: D.L. Schilling, G.R. Lomp, J. Garodnick. "Broadband CDMA Overlay". Proc. 43rd IEEE Vehicular Technologl Conference '93, pp. 452.

[19]: P.A. Ramsdale. W.B. Harrold. "Techniques for Cellular Networks Incorporating Microcells", Proc. IEEE P.I.M.R.C. '92, pp. 169.

[20]: J. Zander. "Performance of Optimum Transmitter Power Control in Cellular Radio Systems", IEEE Transaction on Vehicular technologt: vol.41, No.1, Feb.92. pp. 57.

[21]: L.B. Milstein et. al., "On the Feasibility of CDMA Overlay for Personal Communication Networks". IEEE Journal on Selected Areas on Communications, vol.I0. No.4. May '92. pp. 655.

[22]: K.S. Gilhousen. I.M. Jacobs. R. Padovani. A.J. Viterbi. L.A. Weaver, C.E. Weatly. "On the Capacity of Cellular CDMA System". IEEE Transaction on Vehicular technologl, vol.40, No.2, May'91, pp. 302.

[23]: J.G. Proakis, "Adaptive Equalization for TDMA Digital Mobile Radio". IEEE Transaction on Vehicular technolog?' vol.40, No.2, May '91, pp. 333.

[24]: W.C.Y. Lee, "Overview of Cellular CDMA", IEEE Transaction on Vehicular technologv, vol.40, No.2, May '91, pp. 291.

[25]: Brent R. Peterson, D.D. Falconer, "Minimum Mean Square Equalization in Cyclostationary and Stationary Interference - Analysis and Subscriber Line Calculation", 
IEEE Journal on Selected Areas on Communications, vol.9, No.6. Aug.'91, pp. 931.

[26]: V. Joshi, D.D. Falconer, "Sequence Estimation Techniques for Digital Subscriber Loop Transmission with Cross-talk Interference", IEEE Transaction Communication, vol.38, No.9. Dec.'90, pp. 1367.

[27]: W.A. Gardner, "Introduction to Random Process with Application to Signals and Systems" 2nd edition, McGraw-Hill, 1990.

[28]: J.H. Reed, T.C. Hsia, "The Performance of Time Dependent Adaptive Filters for Interference Rejection", IEEE Transactions on ASSP", vol.38, No.8. Aug. '90, pp. 1373.

[29]: K. Zhou, J.G. Proakis. F. Ling. "Decision Feedback Equalization of Time Dispersive Channels with Coded Modulation". IEEE Transactions Communication. vol.38. Jan.'90, pp. 18.

[30]: T.S. Rappaprot. S.Y. Seidel, R. Singh, " $900 \mathrm{MHz}$ Multipath Propagation Measurements for U.S. Digital Cellular Radiotelephone", IEEE Transactions on Vehicular Technolog: May `90, pp. 132.

[31]: R. Lupas, S. Verdu, "Near Far Resistance of Multiuser Detectors in Asynchronous Channels". IEEE Transactions on Communications, vol.38, No.4, Apr.'90. pp. 496.

[32]: R.L. Lupas. S. Verdu. "Linear Multiuser Detector for Synchronous Code Division Multiple Access Channel". IEEE Transactions on Information Theorn: vol.35. No.1. Jan. 89. pp. 123.

[33]: T.A. Sexton. K. Pahlavan. "Channel Modeling and Adaptive Equalization for Indoor Radio Channels", IEEE Journal on Selected areas on Communications. vol.8. Jan.'89. pp. 114.

[34]: W.A. Gardner, W.A. Brown, "Frequency-Shift Filtering Theory for Adaptive Cochannel Interference Removal". in Proc. of 23rd Asilomar Conf. Sign. Sist. Comp., Pacific Grove, CA. USA. Nov, '89, pp. 562.

[35]: J.S. Lehnert. M.B. Pursley, "Error Probabilities for Binary Direct Sequence Spread Spectrum Communications with Random Signature Sequences”, IEEE Transactions on Communications, vol.35, No.1, Jan.87, pp. 87.

[36]: J.S. Lehnert, M.B. Purshley, "Multipath Diversity Reception of Spread Spectrum Multiple Access Communications", IEEE Transactions on Communications, vol.35. No.11, Nov.87, pp. 1189.

[37]: T. Kanai, Y. Furuya. "A Handoff Control Process for Microcellular Systems" Proc. 37th IEEE Vehicular Technologv Conference, Jun. '87, pp. 170.

[38]: D.C. Cox, "Universal Digital Portable Radio Communication", Proc. of the IEEE. 
vol.75. No.4, Apr.'87, pp. 436.

[39]: S. Verdu, "Optimum Multiuser Asymptotic Efficiency" IEEE Transaction Communication, vol.34, No.8, Sept.'86, pp. 890.

[40]: D.D. Falconer. A.U.H. Sheikh, E. Eleftheriou, M. Tobis. "Comparison of DFE and MLSE Receiver Performance on HF Channels", IEEE Transactions on Communications, vol.33, No.5, May '85, pp. 484.

[41]: G.L. Turin, "The Effects of Multipath and Fading on the Performance of Direct Sequence CDMA Systems", IEEE Journal on Selected Areas on Communications. vol.2. No.4, Jul. '84. pp. 597.

[42]: P. Monsen, "MMSE Equalization of Interference on Fading Diversity Channels", IEEE Transaction Communication, vol.COM-32, '84, pp. 5.

[43]: P.A. Bello, K. Pahlavan, “Adaptive Equalization for Staggered QPSK and QPR over Frequency Selective Microwave Line of Sight", IEEE Transaction Communication. vol.COM-32, 84 , pp. 609.

[44]: G.L. Turin. "Introduction to Spread Spectrum Antimultipath Techniques and their Application to urban digital radio", Proc. of the IEEE, vol.68. No.3. Mar. 80. pp. 328.

[45]: D.V. Sarwate, M.B. Pursley. "Crosscorrelation Properties of Pseudorandom and Related Sequences", Proc. of IEEE, vol.68, No.5, May '80, pp. 593.

[46]: D.D. Falconer, L. Ljung, "Application of Fast Kalman Estimation to Adaptive Equalization”. IEEE Transaction Communication, vol. COM-26. Oct.'78. pp. 1439.

[47]: C.A. Belfiori, J.H. Park, "Decision Feedback Equalization". Proc. IEEE, vol.67. Aug. 79 ,pp. 1143.

[48]: M.B. Pursley, "Performance Evaluation for Phase Coded Spread Spectrum Multiple Access Communication- part I: System analysis". IEEE Transactions on communication, vol.com.25. No.8, Aug.77,pp. 795.

[49]: P. Monsen. “Digital Transmission Performance on Fading Dispersive Channels". IEEE Transactions on Communications, vol.21. No.1. Jan.'73. pp. 33.

[50]: D.C. Cox. "9l0 MHz Urban mobile radio propagation: Multipath Characteristics in New York City", IEEE Transaction on Vehicular technologn, vol.22, No.4. Nov. 73 . pp. 104.

[51]: D.C. Cox, "Delay Doppler Characteristics of Multipath Delay Spread and Average Excess Delay for $910 \mathrm{MHz}$ Urban Mobile Radio Paths", IEEE Transactions on Antennas and Propagation, vol. AP-20, No.5, Sep.'72, pp. 625. 
[52]: G.D. Forney, "Maximum Likelihood Sequence Estimation of Digital Sequences in the Presence of Intersymbol Interferences", IEEE Transaction Information Theory. vol. IT-18, May ·72, pp. 363.

[53]: P. Monsen. "Feedback Equalization for Fading Dispersive Channels". IEEE Transactions on Information Theory, vol.17, No.1, Jan.'71, pp. 56.

[54]: M.E. Austin, "Decision Feedback Equalization for Digital Communication Over Dispersive Channels", MIT Lincoln Lab Technical Report, No.437, Aug.'67.

[55]: Faramarz Hendessi "On the Theory of FRESH-DFE and its Application in Digital Cellular Radio System", Ph.D. Thesis, Dept. of Systems and Computer Engineering. Carleton University, Sept.'94.

[56]: Norm W.K.Lo "Adaptive Equalization for a Multipath Fading Channel in the Presence of Interference". Ph.D. Thesis, Dept. of Systems and Computer Engineering. Carleton University, Apr.' 94.

[57]: M. Abdulrahman, "DFE for Interference and Multipath Suppression in a CDMA System", Ph.D. Thesis. Dept. of Systems and Computer Engineering. Carleton University, Jan.' 94.

[58]: S.R. Chaudry, "Equalization for Multirate Transmission in a CDMA Overlaid Cellular System", M.Eng. Thesis, Dept. of Systems and Computer Engineering. Carleton University."94.

[59]: T.S. Rapapport "Wireless Communications, Principles and Practice" Prentice Hall. 96.

[60]: J.G. Proakis, "Digital Communications". 3rd edition, McGraw' Hill, '95.

[61]: R.L. Peterson, R.E. Ziemer, D.E. Borth, "Introduction to Spread Spectrum Communications", Prentice-Hall, '95.

[62]: W.A. Gardner, S. Venkataraman, "Performance of Optimum and Adaptive Frequency-Shift Filters for Cochannel Interference and Fading", in Proc. of 24th Asilomar Conf. Sign. Syst. Comp., Pacific Grove, CA, USA, Nov, '90. pp. 242.

[63]: W.C. Jacks, "Microwave Mobile Communications", Wiley Interscience, ·74. 


\section{Bibliography}

[1]: Y.C. Hung, "Dynamic Hypermedia Presentations: A Model and its Implementation". IEEE International Conference on Communications, June'97, pp. 805.

[2]: P.A. Ranta and A. Lappetelainen, "Application of Dominant Interference Cancellation in Street Microcells". IEEE International Conference on Communications. June'97, pp. 964.

[3]: S. Sato and Y. Amezawa, "A Study on Dynamic Zone Control for CDMA Mobile Radio Communications", IEEE International Conference on Communications. June'97, pp. 984.

[4]: V. Gondhalekar. R. Jain. and J. Werth, "Scheduling on Airdisks: Efficient Access to Personalized Information Services via Periodic Wireless Data Broadcast". IEEE International Conference on Communications, June'97, pp. 1276.

[5]: A. Hottinen, H. Holma, and A. Toskala, "Multiuser Detection for Multirate CDMA Communications", IEEE International Conference on Communications, June'96. pp. 1819.

[6]: S. Barberis. L. Bonzano. E. Gaiani. and M. Tosalli. "Capacity of a CDMA Wireless Communication System in Picocellular Environment", IEEE International Conference on Communications, June'96, pp. 1824.

[7]: H. Azad, A.H. Aghvami, and W.G. Chambers, "Performance of Multi-Rate Broad Band CDMA in a Fading Channel Using Multi-User/Multi-Path Interference Approximation", IEEE International Conference on Communications. June'96. pp. 1834.

[8]: A. Dabak and K. Kiasaleh, "Integrated Voice and Video Network Using Variable Rate CDMA", IEEE International Conference on Communications, June'96. pp. 1839.

[9]: E. Cohen, and H.-L. Lou, "Multi-Rate Detection for the IS-95A CDMA Forward Traffic Channels Using the $13 \mathrm{kbps}$ Speech Coder", IEEE International Conference on Communications, June'96, pp. 1844.

[10]: C. Su and G. de Veciana, "On the Capacity of Multi-Service Networks", IEEE International Conference on Communications, June'95, pp. 221.

[11]: .C.-L. I and K. K. Sabnani, "Variable Spreading Gain CDMA with Adaptive Control for True Packet Switching Wireless Network", IEEE International Conference on Communications, June'95. pp. 725 
